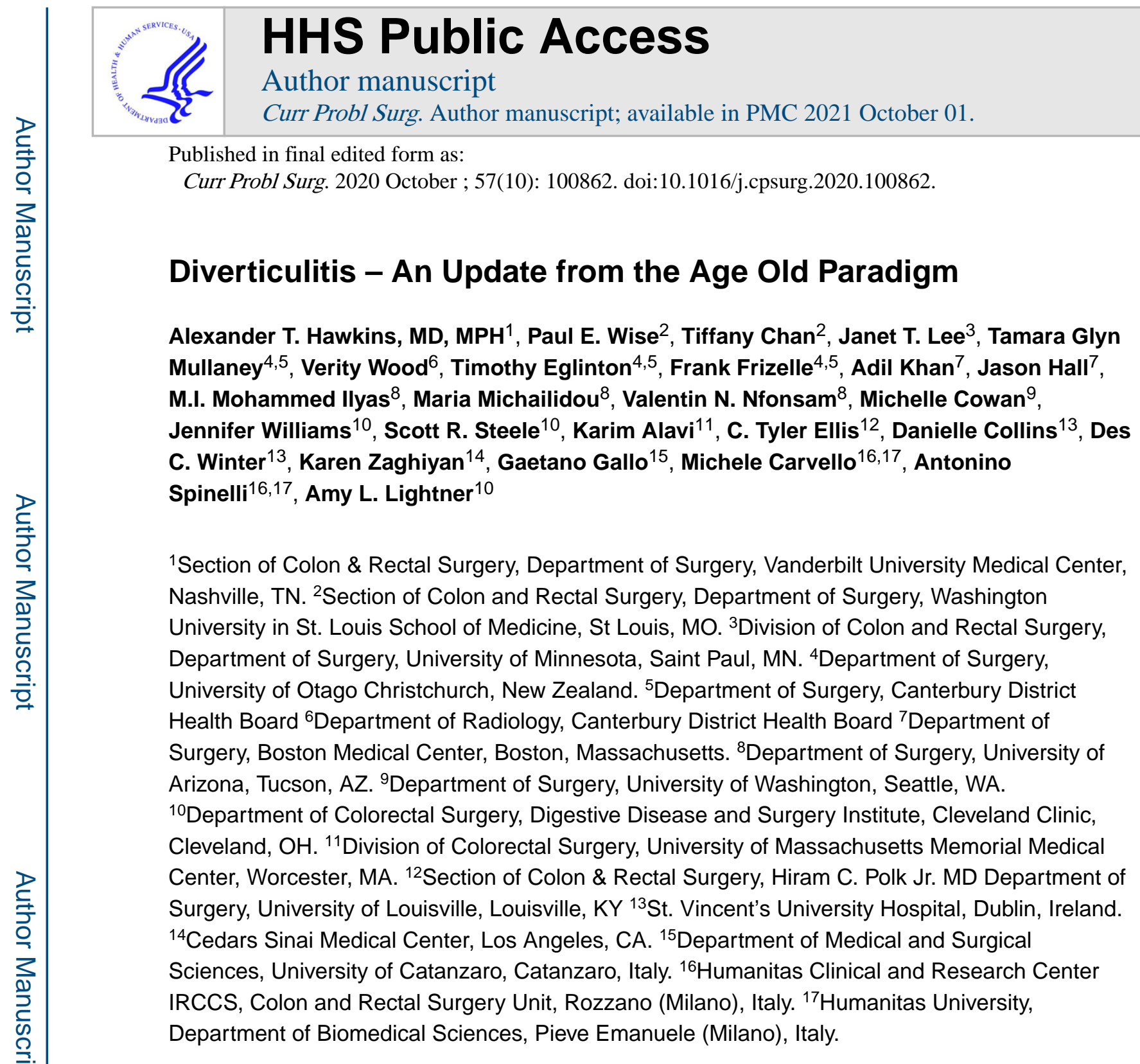

\title{
Monograph In Brief
}

For a disease process that affects so many, we continue to struggle to define optimal care for patients with diverticular disease. Part of this stems from the fact that diverticular disease requires different treatment strategies across the natural history- acute, chronic and recurrent.

To understand where we are currently, it is worth understanding how treatment of diverticular disease has evolved. Diverticular disease was rarely described in the literature prior to the 1900's. In the late 1960's and early 1970's, Painter and Burkitt popularized the theory that diverticulosis is a disease of Western civilization based on the observation that diverticulosis was rare in rural Africa but common in economically developed countries. Previous surgical guidelines focused on

Correspondence: Alexander T Hawkins, MD, MPH, Section of Colon \& Rectal Surgery, Vanderbilt University, 116121 st Ave South, Room D5248 MCN, Nashville, TN 37232, Office: 615.343.4612, Fax: 615.343.4615, alex.hawkins@ vumc.org.

Publisher's Disclaimer: This is a PDF file of an unedited manuscript that has been accepted for publication. As a service to our customers we are providing this early version of the manuscript. The manuscript will undergo copyediting, typesetting, and review of the resulting proof before it is published in its final form. Please note that during the production process errors may be discovered which could affect the content, and all legal disclaimers that apply to the journal pertain. 
early operative intervention to avoid potential complicated episodes of recurrent complicated diverticulitis (e.g., with free perforation) that might necessitate emergent surgery and stoma formation. More recent data has challenged prior concerns about decreasing effectiveness of medical management with repeat episodes and the notion that the natural history of diverticulitis is progressive. It has also permitted more accurate grading of the severity of disease and permitted less invasive management options to attempt conversion of urgent operations into the elective setting, or even avoid an operation altogether.

The role of diet in preventing diverticular disease has long been debated. A high fiber diet appears to decrease the likelihood of symptomatic diverticulitis. The myth of avoid eating nuts, corn, popcorn, and seeds to prevent episodes of diverticulitis has been debunked with modern data. Overall, the recommendations for "diverticulitis diets" mirror those made for overall healthy lifestyle - high fiber, with a focus on whole grains, fruits and vegetables.

Diverticulosis is one of the most common incidental findings on colonoscopy and the eighth most common outpatient diagnosis in the United States. Over 50\% of people over the age of 60 and over $60 \%$ of people over age 80 have colonic diverticula. Of those with diverticulosis, the lifetime risk of developing diverticulitis is estimated at $10-25 \%$, although more recent studies estimate a 5\% rate of progression to diverticulitis. Diverticulitis accounts for an estimated 371,000 emergency department visits and 200,000 inpatient admissions per year with annual cost of 2.1-2.6 billion dollars per year in the United States. The estimated total medical expenditure (inpatient and outpatient) for diverticulosis and diverticulitis in 2015 was over 5.4 billion dollars. The incidence of diverticulitis is increasing.

Besides increasing age, other risk factors for diverticular disease include use of NSAIDS, aspirin, steroids, opioids, smoking and sedentary lifestyle. Diverticula most commonly occur along the mesenteric side of the antimesenteric taeniae resulting in parallel rows. These spots are thought to be relatively weak as this is the location where vasa recta penetrate the muscle to supply the mucosa. The exact mechanism that leads to diverticulitis from diverticulosis is not definitively known.

The most common presenting complaint is of left lower quadrant abdominal pain with symptoms of systemic unwellness including fever and malaise, however the presentation may vary widely. The gold standard cross-sectional imaging is multi-detector CT. It is minimally invasive and has sensitivity between $98 \%$ and specificity up to $99 \%$ for diagnosing acute diverticulitis.

Uncomplicated acute diverticulitis may be safely managed as an out-patient in carefully selected patients. Hospitalization is usually necessary for patients with immunosuppression, intolerance to oral intake, signs of severe sepsis, lack of social support and increased comorbidities. The role of antibiotics has been questioned in a number of randomized controlled trials and it is likely that we will see more patients with uncomplicated disease treated with observation in the future

Acute diverticulitis can be further sub classified into complicated and uncomplicated presentations. Uncomplicated diverticulitis is characterized by inflammation limited to colonic wall and surrounding tissue. The management of uncomplicated diverticulitis is changing. Use of antibiotics has been questioned as it appears that antibiotic use can be avoided in select groups of patients. Surgical intervention appears to improve patient's quality of life. The decision to proceed with surgery is recommended in an individualized manner. 
Complicated diverticulitis is defined as diverticulitis associated with localized or generalized perforation, localized or distant abscess, fistula, stricture or obstruction. Abscesses can be treated with percutaneous drainage if the abscess is large enough. The optimal long-term strategy for patients who undergo successful non-operative management of their diverticular abscess remains controversial. There are clearly patients who would do well with an elective colectomy and a subset who could avoid an operation all together however, the challenge is appropriate riskstratification and patient selection. Management of patients with perforation depends greatly on the presence of feculent or purulent peritonitis, the extent of contamination and hemodynamic status and associated comorbidities. Fistulas and strictures are almost always treated with segmental colectomy.

After an episode of acute diverticulitis, routine colonoscopy has been recommended by a number of societies to exclude the presence of colorectal cancer or presence of alternative diagnosis like ischemic colitis or inflammatory bowel disease for the clinical presentation. Endoscopic evaluation of the colon is normally delayed by about 6 weeks from the acute episode to reduce the risk associated with colonoscopy. Further study has questioned the need for endoscopic evaluation for every patient with acute diverticulitis. Colonoscopy should be routinely performed after complicated diverticulitis cases, when the clinical presentation is atypical or if there are any diagnostic ambiguity, or patient has other indications for colonoscopy like rectal bleeding or is above 50 years of age without recent colonoscopy.

For patients in whom elective colectomy is indicated, it is imperative to identify a wide range of modifiable patient co-morbidities. Every attempt should be made to improve a patient's chance of successful surgery. This includes optimization of patient risk factors as well as tailoring the surgical approach and perioperative management. A positive outcome depends greatly on thoughtful attention to what makes a complicated patient "complicated".

Operative management remains complex and depends on multiple factors including patient age, comorbidities, nutritional state, severity of disease, and surgeon preference and experience. Importantly, the status of surgery, elective versus urgent or emergent operation, is pivotal in decision-making, and treatment algorithms are divergent based on the acuteness of surgery. Resection of diseased bowel to healthy proximal colon and rectal margins remains a fundamental principle of treatment although the operative approach may vary.

For acute diverticulitis, a number of surgical approaches exist, including loop colostomy, sigmoidectomy with colostomy (Hartmann's procedure) and sigmoidectomy with primary colorectal anastomosis. Overall, data suggest that primary anastomosis is preferable to a Hartman's procedure in select patients with acute diverticulitis. Patients with hemodynamic instability, immunocompromised state, feculent peritonitis, severely edematous or ischemic bowel, or significant malnutrition are poor candidates. The decision to divert after colorectal anastomosis is at the discretion of the operating surgeon. Patient factors including severity of disease, tissue quality, and comorbidities should be considered. Technical considerations for elective cases include appropriate bowel preparation, the use of a laparoscopic approach, the decision to perform a primary anastomosis, and the selected use of ureteral stents.

Management of the patient with an end colostomy after a Hartmann's procedure for acute diverticulitis can be a challenging clinical scenario. Between $20-50 \%$ of patients treated with sigmoid resection and an end colostomy after an initial severe bout of diverticulitis will never be 
reversed to their normal anatomy. The reasons for high rates of permanent colostomies are multifactorial. The debate on the best timing for a colostomy takedown continues. Six months is generally chosen as the safest time to proceed when adhesions may be at their softest allowing for a more favorable dissection. The surgical approach will be a personal decision by the operating surgeon based on his or her experience. Colostomy takedown operations are challenging surgeries. The surgeon should anticipate and appropriately plan for a long and difficult operation. The patient should undergo a full antibiotic bowel preparation. Preoperative planning is critical; review the initial operative note and defining the anatomy prior to reversal. When a complex abdominal wall closure is necessary, consider consultation with a hernia specialist. Open surgery is the preferred surgical approach for the majority of colostomy takedown operations. Finally, consider ureteral catheters, diverting loop ileostomy, and be prepared for all anastomotic options in advance.

Since its inception in the late 90's, laparoscopic lavage has been recognized as a novel treatment modality in the management of complicated diverticulitis; specifically, Hinchey III (purulent) diverticulitis. Over the last decade, it has been the subject of several randomized controlled trials, retrospective studies, systematic reviews as well as cost-efficiency analyses. Despite being the subject of much debate and controversy, there is a clear role for laparoscopic lavage in the management of acute diverticulitis with the caveat that patient selection is key.

Segmental colitis associated with diverticulitis (SCAD) is an inflammatory condition affecting the colon in segments that are also affected by diverticulosis, namely, the sigmoid colon. While SCAD is considered a separate clinical entity, it is frequently confused with diverticulitis or inflammatory bowel disease (IBD). SCAD affects approximately $1.4 \%$ of the general population and 1.15 to $11.4 \%$ of those with diverticulosis and most commonly affects those in their 6th decade of life. The exact pathogenesis of SCAD is unknown, but proposed mechanisms include mucosal redundancy and prolapse occurring in diverticular segments, fecal stasis, and localized ischemia. Most case of SCAD resolve with a high-fiber diet and antibiotics, with salicylates reserved for more severe cases. Relapse is uncommon and immunosuppression with steroids is rarely needed. A relapsing clinical course may suggest a diagnosis of IBD and treatment as such should be initiated. Surgery is extremely uncommon and reserved for severe refractory disease.

While sigmoid colon involvement is considered the most common site of colonic diverticulitis in Western countries, diverticular disease can be problematic in other areas of the colon. In Asian countries, right-sided diverticulitis outnumbers the left. This difference seems to be secondary to dietary and genetic factors. Differential diagnosis might be difficult because of similarity with appendicitis. However accurate imaging studies allow a precise preoperative diagnosis and management planning. Transverse colonic diverticulitis is very rare accounting for less than $1 \%$ of colonic diverticulitis with a perforation rate that has been estimated to be even more rare. Rectal diverticula are mostly asymptomatic and diagnosed incidentally in the majority of patients and rarely require treatment. Giant colonic diverticula (GCD) is a rare presentation of diverticular disease of the colon and it is defined as an air-filled cystic diverticulum larger than $4 \mathrm{~cm}$ in diameter. The pathogenesis of GCD is not well defined.

Overall, the management of diverticular disease depends greatly on patient, disease and surgeon factors. Only by tailoring treatment to the patient in front of us can we achieve optimal outcomes. 


\section{Introduction}

Regardless of how the problem is examined, diverticular disease is a significant burden both patients and health care systems. In Western and industrialized nations, diverticulosis of the sigmoid colon, defined by the sac-like protrusion of the colonic wall, has prevalence rates between 5 and $45 \%$, depending on the age and method of diagnosis. ${ }^{1}$ Approximately 4 to $15 \%$ of patients with diverticulosis develop diverticulitis. ${ }^{2-4}$ Complications lead to substantial morbidity and mortality. For perforation alone, the 1-year mortality is $19 \% .{ }^{5}$ In 2004, diverticular disease was responsible for 313,000 hospitalizations, 1.9 million ambulatory care visits, and 3365 deaths in the United States. ${ }^{6}$ Spending on diverticular disease in the United States has been estimated at 2.5 billion dollars per year. ${ }^{7}$ In 2004 , there were an estimated 2.8 million prescriptions at a cost of $\$ 100$ million filled at retail pharmacies for diagnosis of diverticular disease. ${ }^{6}$ Between 1998 and 2005, US hospital admissions for diverticulitis increased by $26 \%$ and elective operations by $29 \%{ }^{8}$

An attempt to capture the entire spectrum of management of diverticular disease is an ambitious one. A disease process that spans both the acute and chronic, the mild and severe and the young and the old is difficult to define in a single reading. But in this paper, we lay the foundation for the management of the breath of diverticular disease. Decades of research inform our current best practices. Details on these follows below.

\section{Diverticulitis: a historical perspective}

\section{Introduction}

The precise underlying etiologies of diverticular disease, risk factors for acute episodes, and diets that may prevent disease occurrence have long been the subject of debate. Diverticular disease was rarely described in the literature prior to the 1900's. In the late 1960's and early 1970's, Painter and Burkitt popularized the theory that diverticulosis is a disease of Western civilization based on the observation that diverticulosis was rare in rural Africa but common in economically developed countries, ${ }^{9,10}$ though critics will note that in their original paper they assessed neither diet nor presence of diverticulosis to support this claim. Their subsequent studies on colonic pressure, ${ }^{11}$ stool weight, and colonic transit times ${ }^{12}$ to substantiate their hypotheses have also been challenged.

The first description of surgical management of diverticulitis was by Mayo, who outlined the operations and pathologic findings for a series of five patients in $1907 .{ }^{13}$ In his initial papers, Mayo also briefly outlined surgical options for diverticulitis including abscess drainage, and staged operations such as diverting colostomy with or without subsequent resection and eventual colostomy closure. ${ }^{14}$ This was followed by the three-stage operation proposed by Rankin and Brown ${ }^{15}$, which subsequently has been superseded by the twostage operation eponymous with Henri Hartmann, who first proposed the procedure in 1921 for the management of sigmoid carcinoma. ${ }^{16}$

The evolution in our understanding of the natural history of diverticulitis has altered medical and surgical management recommendations over the course of the last 50 years. Despite the debunking of old surgical dogma, modern perspectives have yet to completely translate to 
the realm of public knowledge. In this section common myths and misconceptions will be discussed.

\section{Diverticulitis: when to cut it out?}

Previous surgical guidelines focused on early operative intervention to avoid potential complicated episodes of recurrent complicated diverticulitis (e.g., with free perforation) that might necessitate emergent surgery and stoma formation. This included recommendations for routine elective resection after two attacks in patients over 50 years of age and after one attack in patients younger than 50 years of age. The premise of these recommendations had underlying assumptions that include the following: 1) patients who had already experienced more than one prior episode would be less responsive to medical therapy, lead to complicated diverticulitis, need for emergent operation/stoma, and thus have a higher mortality risk, and 2) young patients would have a longer potential time to experience a recurrence and its associated sequelae.

\section{How many episodes is too many episodes?}

In the late 1960's, Parks found that in a cohort of 455 patients admitted for treatment of diverticulitis, 317 were treated medically on the first admission. Patients included in this series had clinical and radiologic evidence of diverticulitis on barium enema and/or evidence of local diverticular inflammation at the time of operation or necropsy. Of patients treated medically, $24.6 \%$ (78 patients) were subsequently readmitted with a second attack, 3.8\% with a third attack, and $1.6 \%$ with a fourth attack. Of these recurrent episodes, $46 \%$ occurred within the first year. Because 20 of the 78 patients (25.6\%) with recurrent attacks ultimately needed surgery, and the mortality rate associated with the second attack was $7.7 \%$ compared to only $3 \%$ in the first attack, Parks concluded that medical treatment of repeat episodes was less likely to be successful. ${ }^{17}$ This observation formed some of the basis for recommending early surgery after one or two episodes to prevent future attacks.

As of the 1990's, both the American Society of Colon and Rectal Surgery ${ }^{18}$ as well as the European Association for Endoscopic Surgery ${ }^{19}$ supported the recommendation for elective resection after two episodes of uncomplicated diverticulitis. However, criticism of this recommendation began to build as newer studies challenged dogma founded on the limited data from more than 20 years prior. "Modern" series have been published in the era of more advanced diagnostic imaging usually with computed tomography (CT) scanning, routine use of image-guided percutaneous drainage, and advances in the medical management of sepsis. This more recent data has challenged prior concerns about decreasing effectiveness of medical management with repeat episodes and the notion that the natural history of diverticulitis is progressive. It has also permitted more accurate grading of the severity of disease and permitted less invasive management options to attempt conversion of urgent operations into the elective setting, or even avoid an operation altogether.

\section{The role of "early surgery" to prevent future complicated diverticulitis}

Contrary to prior dogma, complicated diverticulitis appears to present more commonly in patients who had only had one or two attacks, ${ }^{20}$ and patients with more than two episodes were not at increased risk for poor outcomes. In other words, patients who had experienced 
more than two episodes were actually less likely to experience free perforation, had a lower rate of requiring diverting ostomy, and higher rates of successful resection with primary anastomosis. ${ }^{21}$ Therefore, in the absence of feculent peritonitis, when patient physiology, comorbidities, and local technical expertise permits, non-operative management of perforated diverticulitis presenting with abscess or even locules of free air has been demonstrated to be highly successful and feasible in the majority of patients. ${ }^{22}$

The decision to offer surgery for other manifestations of complicated diverticulitis such as free perforation, fistula, or obstruction, is generally straightforward. However, some debate still remains about what to do with the diseased colonic segment following resolution of an episode of complicated diverticulitis with abscess treated with or without percutaneous drainage. Data that supports the recommendation for elective colectomy after diverticulitis with abscess is based on concerns that recurrence rates can be as high as $40 \% .{ }^{23,24} \mathrm{~A}$ recent review of a statewide database in New York suggests that many patients could be successfully managed non-operatively without routine colectomy after their first presentation of diverticular abscess. Based on more than 10,000 admissions for initial diverticular abscess, observation without elective colectomy was associated with lower stoma rates, fewer inpatient hospital days, but higher costs. Of this cohort, $31 \%$ failed initial non-operative management, $16 \%$ underwent elective colectomy within 6 months of discharge, and $52 \%$ of patients continued with nonoperative management. In this latter group, overall recurrence at 5 years was $25 \%$ (and only $13 \%$ if the patients made it beyond 1 year without recurrence).

\section{Role of routine elective resection for young patients}

Routine elective resection based on age $<50$ is no longer recommended. More recent studies have allayed concerns that diverticulitis is more virulent in younger patients, ${ }^{25}$ and therefore routine resections are no longer recommended to prevent the possibility of recurrence requiring emergency colectomy and/or colostomy. However, in a large statewide database study by Anaya and Flum in 2005 that included more than 20,000 patients treated without initial operation, the overall recurrence rate was $19 \%$, but the rate was $27 \%$ in patients under 50 years of age versus $17 \%$ in those over $50(\mathrm{p}<0.001)$. While the overall rate of emergency colectomy or colostomy was only $5.5 \%$, it was also significantly more common in younger patients $\left(7.5\right.$ vs. $5.0 \%$, p<0.001). ${ }^{20}$ However, a contemporary study in 2004 by Guzzo and Hyman demonstrated that the risk of requiring surgery at the initial admission was similar between older (22\%) and younger patients $(24 \%, \mathrm{p}=0.8)$. There was a low rate of recurrence following successful medical management, with perforation risk of only $0.5 \%$, suggesting that the role of performing routine resection in young patients to prevent emergent colectomy in the future may not be warranted. ${ }^{26}$ While virulence appears the same across age groups, the potentially longer time course over which disease can recur should still be taken into consideration when discussing surgery with young patients.

Cost-benefit modeling has been performed in both patients over and under 50 years of age, and also speak against the prior recommendation to routinely offer resections to young patients. One study utilizing Markov modeling of elective resections after two episodes versus medical therapy show lower quality adjusted life years associated with routine 
resection after two episodes. ${ }^{27}$ When similar modeling was performed looking at deferring elective colectomy until after a fourth attack, the finding was that expectant management was the dominant strategy in both young (age 35) and older (age 50) hypothetical cohorts. Notably, a very small proportion $(0.3 \%)$ of patients would actually develop a fourth attack of diverticulitis. Deferring surgery until the fourth episode in patients over 50 resulted in only a marginal decrease in mortality of $0.5 \%, 0.7 \%$ fewer colostomies, and cost savings of approximately US $\$ 1000$ compared to colectomy after the first episode. ${ }^{28}$ Similarly in younger patients, colectomy after the fourth episode resulted in $0.1 \%$ fewer deaths, $2 \%$ fewer colostomies, and cost savings of US $\$ 5429$ per patient.

Overall, the practice of recommending elective colectomy to prevent future recurrence (regardless of age) out of the fear of need for emergency surgery and associated stoma formation is generally discouraged as the available data does not support this practice. The estimated rate of requiring urgent Hartmann's procedure is 1 in 2000 patient-years of follow up. ${ }^{29}$ Given that elective surgery carries its own set of complications and risks, more recent evidence has therefore led to a modification in the ASCRS guidelines stating that the "... decision to recommend elective sigmoid colectomy... should be individualized", depending on the medical condition of the patient, frequency and severity of attacks, and persistence of symptoms. ${ }^{30}$

\section{Let food be thy medicine-fiber, seeds, nuts, and other diverticular diet lore}

The role of diet in preventing diverticular disease has long been debated. Even 40 years ago, there was some postulation that high fiber diets may actually reduce the incidence of diverticular disease. This has been supported by observational studies noting that populations that adopted more "Western" diets with refined grains and decreased fiber had higher rates of diverticular disease. ${ }^{10,12}$ Specifically, researchers have tried to establish the relationship between high fiber diets and the development of diverticulosis, the role of specific foods such as nuts and seeds in the precipitation of diverticulitis, and the role of specific diets in the management and treatment of diverticulitis during an acute episode.

\section{Fiber - an ounce of prevention?}

The Dietary Guidelines for Americans (2015-2020) recommends a dietary fiber intake of 14 grams per 1,000 calories consumed. For example, for a 2,000-calorie diet, the fiber recommendation is 28 grams per day. ${ }^{31}$ However, it is difficult to find the originating source for these recommendations. The exact role that fiber plays in the pathogenesis of asymptomatic and symptomatic diverticular disease is still a subject of debate. While population-based studies such as the Million Women Study (MWS) ${ }^{32}$ and the European Prospective Investigation into Cancer and Nutrition (EPIC) ${ }^{33}$ suggest that a high fiber diet protects against diverticula becoming symptomatic, cross-sectional studies looking at asymptomatic diverticula identified on colonoscopy did not demonstrate that lower fiber diets or constipation were risk factors for developing diverticulosis ${ }^{34}$ and did not identify a protective role of a high fiber diet. ${ }^{1}$ It is possible that the reason for this conflicting evidence is due to study design and the variation in endpoints measured. Interestingly, there are no studies that clearly address whether fiber supplements (as opposed to dietary fiber) reduces the risk of diverticulitis attacks. ${ }^{35}$ However, as many people find it difficult to obtain the 
recommended intake of fiber through diet alone, supplementation is a low-risk method of increasing intake. On balance, given that a high fiber diet appears to decrease the likelihood of symptomatic diverticulitis, and may confer other health benefits beyond the management of diverticular disease, the recommendation is for patients to adhere to a high fiber diet.

\section{Fiber intake and diverticular disease}

Multiple prospective, population-based studies have looked at aspects of diets and their association with diverticular disease. Supporting the comments above, the MWS, based out of the UK, surveyed 1.3 million women aged 50-65 years regarding social, demographic, and lifestyle factors and were then linked to hospital records to confirm rates of symptomatic diverticular disease. ${ }^{32}$ This study demonstrated that high intake of dietary fiber was associated with a reduced risk of diverticular disease. Particularly, a diet high in fruit and cereals was most protective. The relative risk of diverticular disease per $5 \mathrm{~g} /$ day of fiber intake was 0.86 (95\% CI, 0.84-0.88). The mean total intake of dietary fiber was 13.8 grams per day, which is still less than half the current recommended daily intake of 25-30 grams per day.

Similarly, the EPIC-Oxford study examined the association between fiber intake and admission to hospital or death from diverticular disease in more than 47,000 people over an average period of 11.6 years. Participants in the highest quintile of fiber intake ( $\geq 25.5 \mathrm{~g} / \mathrm{day}$ for women and $\geq 26.1 \mathrm{~g} /$ day for men) had a lower relative risk (0.59, 95\% CI 0.46-0.78, $\mathrm{P}<0.001)$ of both complicated and uncomplicated diverticular disease compared with those in the lowest quintile ( $<14 \mathrm{~g} /$ day for both women and men). Vegetarians made up 33\% of the subjects and demonstrated an additional $31 \%$ lower risk of diverticular disease compared to meat eaters. ${ }^{36}$

\section{Nuts, seeds, and diverticulitis}

Patients with diverticular disease commonly ask whether they should avoid eating nuts, corn, popcorn, and seeds to prevent episodes of diverticulitis. The rationale for this was that these substances tend to pass through the gastrointestinal tract almost intact and would therefore be of the perfect shape and size for getting lodged in a diverticulum, presumably resulting in diverticulitis. ${ }^{37}$ In reality, the precise mechanism for precipitating diverticulitis is likely more complex and still not completely understood. While this recommendation seems to make theoretical sense, this dietary urban legend has essentially been debunked. ${ }^{38}$ In the Health Professionals Follow-up Study (HPFS), a cohort of more than 47,000 men were surveyed regarding food intake frequency. There was actually an inverse association between nut and popcorn assumption and risk of diverticulitis, defined as abdominal pain and one of the following: 1) fistula, abscess, perforation, or obstruction; 2) treatment with antibiotics, hospitalization, or surgery; or 3) severe or acute or presenting with fever, requiring medication, or evaluated with CT scan. Self-reports of these symptoms were then corroborated with medical records. Men who ate these foods at least twice per week were actually less likely to develop diverticulitis, even when controlling for other potential risk factors including age; body mass index; dietary fat, fiber, and red meat consumption; physical activity; cigarette smoking; and NSAID and acetaminophen use. There was no association between the development of diverticular bleeding or uncomplicated 
diverticulosis. In particular, insoluble fiber or cellulose appeared to have the strongest protective effect. ${ }^{39}$

\section{Low fiber/low residue diets and acute diverticulitis}

Traditionally, many caregivers have advocated for a low residue diet during an acute episode of diverticulitis once a liquid diet has been tolerated without pain or alteration in clinical status. The term "low residue" diet has fallen out of favor as the term is non-specific and difficult to quantify. ${ }^{40}$ Instead, the suggested terminology is a "low fiber" diet, generally defined as $<10 \mathrm{~g} /$ day. ${ }^{41,42}$ Currently in the literature the terms low residue and low fiber may be used interchangeably, however. Conceptually, this dogma of low fiber diets with diverticulitis would be based on a low fiber diet theoretically reducing the frequency and volume of stools to ease passage through the inflamed segment allowing the inflammation to improve more quickly. Once the acute episode has resolved, the general recommendation, as described above, would be to then gradually resume a high fiber diet to minimize further attacks. However, the evidence for adhering to a low fiber diet during an acute episode is based on scant evidence, ${ }^{43}$ so this dogma remains without scientific evidence.

\section{Summary}

Overall, the recommendations for "diverticulitis diets" mirror those made for overall healthy lifestyle - high fiber, with a focus on whole grains, fruits and vegetables. Many people who develop asymptomatic diverticulosis will not develop diverticulitis and its potential associated sequelae. In those who do develop symptoms, the attitude towards early surgical management is shifting to more expectant management with delay of definitive surgery. Furthermore, the focus of surgical decision-making is moving away from dogmatic recommendations in favor of shared decision-making between patient and surgeon, balancing severity of symptoms, patient lifestyle, age, and morbidity.

\section{Epidemiology/Classification/Pathophysiology of Diverticulitis - Janet Lee}

\section{Epidemiology}

Diverticulosis is one of the most common incidental findings on colonoscopy ${ }^{6}$ and the eighth most common outpatient diagnosis in the United States. ${ }^{44}$ Over $50 \%$ of people over the age of 60 and over $60 \%$ of people over age 80 have colonic diverticula. ${ }^{44,45}$ Of those with diverticulosis, the lifetime risk of developing diverticulitis is estimated at $10-25 \%,{ }^{46}$ although more recent studies estimate a $5 \%$ rate of progression to diverticulitis. ${ }^{2}$ Diverticulitis accounts for an estimated 371,000 emergency department visits and 200,000 inpatient admissions per year with annual cost of 2.1-2.6 billion dollars per year in the United States. ${ }^{44,47}$ The estimated total medical expenditure (inpatient and outpatient) for diverticulosis and diverticulitis in 2015 was over 5.4 billion dollars. ${ }^{47}$

Painter and Burkitt first observed that diverticulosis is more common in urbanized/ industrialized areas than rural Africa. ${ }^{10}$ The paucity of fiber in the Western diet has been linked to higher rates of diverticulosis. A low fiber diet results in less stool volume, decreased colon diameter, increased intraluminal pressure and increased formation of diverticula. Increased industrialization and urbanization and greater spread of the Western 
diet have led to higher rates of diverticular disease in other parts of the world such as Asia and Africa. ${ }^{48,49}$ White people have the highest prevalence of hospitalization for diverticulitis $(62 / 100,000)$ versus African Americans or Hispanics $(30 / 100,000)$ and Asians $(10 / 100,000) .{ }^{50}$ In large database studies of diverticulitis, Whites comprise $60-70 \%$ of hospitalized patients with diverticulitis. ${ }^{8}$ In Asian countries, diverticulitis is much less common that in Western countries, but when it does occur, it more frequently affects the right side of the colon. If Asian patients move to Westernized areas, however, the risk of diverticular disease and left sided disease increases. Autopsy studies have shown a $1 \%$ prevalence of diverticulosis in Japanese who live in Japan, but a 50\% prevalence of Japanese living in the United States. ${ }^{51}$

The incidence of diverticulitis is increasing. Wheat and Strate ${ }^{50}$ performed a retrospective review of the Nationwide Inpatient Sample from 2000 through 2010 analyzing trends in hospitalization for diverticulitis and diverticular bleeding. Over the 10-year study period there were over 2.1 million hospitalizations for diverticulitis with an average of 195,000 per year. The number of admissions for diverticular bleeding decreased over the study period while admissions for diverticulitis increased over the 10 years, with a peak in 2008. ${ }^{50}$

Lee et $\mathrm{al}^{52}$ also performed a retrospective review to identify trends in diverticulitis. Using data from the Rochester Epidemiology Project, they looked at changes in demographics of patients between 1980 and 2007 who developed diverticulitis. They found a significant increase in incidence of diverticulitis between decades (1980-1989 vs. 1990-1999 vs. 2000$2007, \mathrm{p}<0.001)$. The authors estimate an increase of 50\% between 1990-1999 and 20002007. The authors also found that visceral and subcutaneous adipose tissue were independently associated with diverticulitis, suggesting that distribution of body weight and fat (ie central obesity) was more closely responsible for increasing rates of diverticulitis. 52

Diverticular disease is more common in middle-aged to older individuals, and its prevalence increases with age as well. By age $40,5 \%$ of the population has diverticulosis, while the prevalence may be as high as $60-80 \%$ by age 80 . Recent studies have shown that rates of diverticulitis in young people is increasing as well. Bharucha et al ${ }^{53}$ found that individuals aged 40-49 have had a 132\% increase in incidence from 1980 through 2007. Etzioni et al ${ }^{8}$ also performed a retrospective review of the changing demographics of patients hospitalized for diverticulitis between 1998 and 2005. Among patients aged 18-44, the rates of admission for diverticulitis increased by $82 \%$ and number of elective operations increased by $73 \%$ over the study period. In contrast, rates of admission for diverticulitis in the 65-74 year-old group remained stable, and rates of admission for diverticulitis decreased in the 75+ year old group. ${ }^{8}$

In younger age groups, diverticular disease is more common in men than women, with an estimated 2-3:1 ratio. After the 6th decade, however, the prevalence is more similar. ${ }^{50,53}$ Women make up a higher proportion of hospitalized diverticulitis patients ${ }^{8,50}$ and have a higher incidence of fistula formation. Men are more likely to have bleeding from diverticular disease. 
Other risk factors for diverticular disease include use of NSAIDS, aspirin, steroids, opioids, smoking and sedentary lifestyle. Family history and genetics is also an important risk factor for development of diverticulitis. Twin studies have shown that the odds of developing diverticular disease if a co-twin has the disease is much higher in monozygotic twins than dizygotic twins. ${ }^{54,55}$ Using statistical modeling, studies have shown that genetics accounts for $40-50 \%$ of risk for diverticular disease while environmental effects account for $50-60 \%$ of the trait variability. ${ }^{54,55}$

\section{Classification}

Diverticulitis may be classified as either uncomplicated or complicated. The vast majority of patients will have uncomplicated diverticulitis $(\sim 90 \%)$ with a typical presentation of fever, abdominal pain and/or leukocytosis. Complicated diverticulitis may present with abscess, fistula, perforation, stricture, or peritonitis. Abscess (70\%) is the most common manifestation of complicated diverticulitis. ${ }^{53}$

Diverticulitis can also be classified as acute or chronic. Chronic diverticulitis can present as recurring, intermittent episodes of acute disease, or persistent, symptomatic inflammation; so-called smoldering diverticulitis. The 10-year risk of recurrence of diverticulitis is $20 \%$ after one episode. After 2 episodes of diverticulitis, the 1-year risk is $20 \%$. Hall et al ${ }^{56}$ performed a retrospective review of all diverticulitis patients over a 6-year period with a CTdocumented episode of diverticulitis and analyzed risk factors for recurrence. In this study of 672 patients, the mean follow-up was $42.8+/-24$ months. The vast majority of patients had uncomplicated diverticulitis while $8.9 \%$ presented with complicated disease. The overall recurrence rate at 5 years was $36 \%$. Complicated recurrence was 3.9\%. Risk factors for recurrent diverticulitis included irritable bowel syndrome, complicated presentation, previous hysterectomy, and family history of diverticulitis. Family history, length of involved colon and retroperitoneal abscess were independently statistically significant risk factors for recurrent disease. ${ }^{56}$

Other newer terms have been used to classify diverticular disease including segmental colitis associated with diverticulosis (SCAD) and symptomatic uncomplicated diverticular disease (SUDD) ${ }^{57,58}$ (Table 3). SCAD is different than diverticulitis in that a nonspecific inflammation of the colon is seen in an area of diverticula, but the diverticular orifice itself is not involved. ${ }^{59} \mathrm{SCAD}$ is typically benign and self-limited. It may present with rectal bleeding, diarrhea or abdominal pain. Histologically and endoscopically, SCAD may resemble inflammatory bowel disease. SUDD is characterized by chronic abdominal pain in absence of acute symptoms, radiographic evidence, or endoscopic evidence of diverticulitis or colitis. ${ }^{58}$ SUDD is similar to irritable bowel syndrome as both involve visceral hypersensitivity and changes in colonic motility. ${ }^{60}$

The most well-known classification schema for acute diverticulitis is the Hinchey criteria (Table 1). First described by Hinchey et al in 1978, the Hinchey criteria classify the disease based on extent of inflammatory complications. ${ }^{61}$ These classification schema help identify patients that are more likely to have recurrent attacks or require surgical intervention after the initial presentation of diverticulitis. Ambrosetti ${ }^{23}$ developed radiologic criteria to help guide clinical management based on CT findings (Table 2a). Using this criteria, moderate 
diverticulitis is defined by localized wall thickening and inflammation of pericolic fat. Severe diverticulitis is defined by wall thickening as well as inflammation, abscess, extraluminal air or contrast. The modified Hinchey classification (Table 3) has been applied to preoperative CT findings as well to help guide management. ${ }^{62}$ The modified Hinchey criteria correlates with postoperative morbidity and mortality and is also predictive of recurrence when managed nonoperatively. ${ }^{24}$ The Hinchey classification, however, is limited, however, as the presence of purulent or feculent peritonitis can only be determined at the time of surgery.

\section{Pathophysiology}

Diverticula most commonly occur along the mesenteric side of the antimesenteric taeniae resulting in parallel rows. These spots are thought to be relatively weak as this is the location where vasa recta penetrate the muscle to supply the mucosa. Colon diverticula are false diverticula and contain only mucosal and muscularis mucosal layers. The reason behind their formation is unclear with the most common theories involving structural abnormalities of the colon wall, disordered motility and fiber deficiencies. Studies have shown thickening of the circular muscle, shortening of the taenia, and narrowing of the lumen in patients with diverticular disease compared with those with normal colons. ${ }^{63}$ The thickened circular muscle in patient with diverticular disease is the result of increased elastin deposition as opposed to muscular hypertrophy or hyperplasia, with over $200 \%$ increase of elastin in patients compared with controls. ${ }^{64}$ Studies have also shown that patients with diverticular disease have greater rates of collagen cross-linking. ${ }^{65}$ Increased collagen cross-linking may cause the tissues to become stiffer leading to a loss in compliance of the colonic submucosa, making it more susceptible to injury. Collagen cross-linking increases with age, and thus, may explain why the prevalence of diverticular disease increases with age. Changes in collagen cross-linking and elastin deposition may also explain why patients with connective tissue disorders are also more prone to diverticulosis earlier in life.

In addition to changes in structure and composition of colonic tissue, patients with diverticulosis also have higher intraluminal pressures in the sigmoid colon versus controls. 66 This can lead to increased risk of injury to the mucosa with high intraluminal pressures. Studies have also shown that patients with diverticular disease have disordered colonic motility in the descending and sigmoid colon with disorganized, frequent retrograde propagation of propulsions. ${ }^{60} \mathrm{In}$ a high fiber diet, motility is faster and intraluminal pressures are lower, which may explain why a high fiber diet is protective against diverticular disease.

The exact mechanism that leads to diverticulitis from diverticulosis is not definitively known. Diverticulitis is commonly believed to be caused by obstruction of a diverticulum, leading to stasis, ischemia, microperforation and infection. ${ }^{67}$ The microperforation then leads to a characteristic inflammatory response of varying degrees. Development of an abscess, phlegmon, fistula or free perforation may then follow. Inflammation in the surrounding tissues can then lead to altered gut function and change in bowel function.

More recent studies suggest other potential contributing factors including chronic inflammation, the gut microbiome, and genetics. ${ }^{54,55,68-72}$ Risk factors for other known 
chronic inflammatory diseases such as cardiovascular disease and diabetes are also risk factors for diverticulitis. Increased dietary fiber may be protective against diverticulitis by altering the microbiome. Patients with diverticular disease have a different composition in the intestinal microbiome than controls, with higher levels of Bifidobacterium. ${ }^{71}$ Other studies have found differences in composition of patients with active diverticulitis compared with controls with diverticulosis. ${ }^{69,70}$ The correlation or causation these potential contributors to the development of diverticulitis is unknown.

Schafmayer et al ${ }^{72}$ recently performed a genome-wide association study looking at genetic risk factors for diverticulosis and diverticulitis. Using genotypes from a large Biobank of patients of European descent, they compared loci of cases and controls. They found that candidate genes in case with diverticulosis were related to intestinal neuromuscular function and impaired connective fiber support. Identified candidate genes for cases with diverticulitis were related to epithelial dysfunction. This study suggests a genome-specific reason for the development of diverticular disease and diverticulitis and may help identify those at higher risk of developing symptoms or complications

\section{Summary}

The incidence of diverticulitis is increasing, and the age of the average patient is decreasing. The change in the Western diet, inflammation, and perhaps gut microbiome may have some relation to the evolution of the disease. Understanding the exact mechanism of diverticular formation or transformation from diverticulosis to diverticulitis may help guide future disease prevention and treatments.

\section{Presentation, Preferred Imaging and Criteria for Outpatient Management Introduction}

The epidemiology of diverticular disease has been evolving over the past century. ${ }^{73}$ Currently, $70 \%$ of patients over the age of 80 years have diverticulosis, and there is an increasing incidence of younger patients with symptomatic disease. ${ }^{74}$ Findings from screening colonoscopy suggest a progressive disorder, supported by the increased prevalence and density of diverticula with age. ${ }^{75}$ Of those with diverticular disease, recent studies suggest 3-7\% may develop acute diverticulitis (AD). ${ }^{2,76}$

National database studies suggest a continuing increase in emergency presentations for AD and the associated costs over the past two decades. ${ }^{73,74,77,78}$ The aging population may explain some of this, however an exponential rise in the prevalence in younger age categories and other demographic groups (specifically women) have also been implicated.

In the western population diverticulosis is predominantly left sided (86\%), particularly affecting the sigmoid colon, with the right side or whole colon less commonly affected, and symptomatic disease also following this distribution. ${ }^{75}$ In Asian communities however right sided disease predominates, and may be confused with appendicitis. Acute diverticulitis may be defined as uncomplicated or complicated (approximately 15-35\% of presentations) in the presence of perforation, abscess, fistulisation or obstruction. ${ }^{79-81}$ The vast majority (>80\%) of acute presentations with $\mathrm{AD}$ are now managed non-operatively. ${ }^{77,82}$ 


\section{Presentation}

The most common presenting complaint is of left lower quadrant abdominal pain with symptoms of systemic unwellness including fever and malaise, however the presentation may vary widely. The differential diagnosis is broad and includes appendicitis and malignancy (among others). Initial assessment aims to establish the diagnosis of AD, the presence of (or risk of developing) complications and the best course of treatment.

A routine history should focus on duration and details of illness and risk factors for malignancy or other differential diagnoses. Clinical examination should establish the presence and degree of sepsis and the presence and extent of peritonism. Standard laboratory testing includes a complete blood count, urea, electrolytes and inflammatory markers, looking for evidence of inflammatory response and effect on other organ systems. ${ }^{30,83}$ However, normal laboratory testing does not preclude a diagnosis of AD. ${ }^{84} \mathrm{Clinical}$ assessment in conjunction with laboratory testing can be reasonably accurate, ${ }^{85}$ however there is considerable overlap of symptoms with other diseases ${ }^{29}$ and as such radiologic investigation is essential for an accurate diagnosis.

\section{Preferred Imaging}

Plain Film—Plain radiography of the abdomen may demonstrate gross free air or obstruction, however it lacks sensitivity and specificity and therefore does not have a routine role in the evaluation of $\mathrm{AD} .{ }^{86} \mathrm{~A}$ plain radiograph of the chest may be diagnostically useful if the differential diagnosis includes pneumonia or as part of the peri-operative work-up if surgery is deemed necessary.

Contrast Enema-The initial investigation of choice prior to the advent of computed tomography (CT) was contrast enema. While many earlier studies linked the presence of left iliac fossa symptoms and subsequent barium enema showing diverticulosis as evidence the patient had diverticulitis, subsequent reviews cast doubt on the reliability of this assumption. 29

Barium enema is not often used in the acute situation as barium can cause peritonitis and obscure views if a subsequent $\mathrm{CT}$ is required. Water soluble contrast has been used as an adjunct to primitive $\mathrm{CT},{ }^{87}$ however it is not now commonly used to diagnose $\mathrm{AD}$ as it has been surpassed in sensitivity and speed by the latest iterations of CT technology.

Contrast enema gives good luminal images but does not reflect the peri-colonic process. It may have a role in the identification and anatomic mapping of structuring or fistulous disease, however this has largely been surpassed by the use of CT with rectal contrast. ${ }^{88}$

Ultrasound-Ultrasound (US) has been proposed as an alternative or adjunctive imaging modality without the potential harm of radiation exposure. ${ }^{89,90}$ Proponents advocate a role in diagnosis and monitoring of uncomplicated diverticulitis ${ }^{89}$ and possibly in intervention for complicated disease. The German guidelines recommend a 'Qualified abdominal US' as the first line imaging investigation for uncomplicated AD. ${ }^{86}$ The defining features of $\mathrm{AD}$ on US depend on the stage of the disease and include colonic wall thickening and peridiverticular inflammation appearing as non-compressible, hyperechoic fat which is tender to 
probe pressure. ${ }^{89,90}$ Complicated disease such as abscesses may be demonstrated on US and this used to guide drainage.

Ultrasound is user dependent, has a tendency to underestimate the stage of the disease and sensitivity may depend on the location of the disease (for example deep mesenteric and pelvic disease is better imaged by $\mathrm{CT}$ ) and patient body habitus. In experienced hands using a targeted approach, sensitivity and specificity approach $98 \% .{ }^{86}$ A meta-analysis found it comparable to $\mathrm{CT}$ in the accuracy of diagnosis of $\mathrm{AD}$, however it is less likely to identify other sources of pathology. ${ }^{91}$ It may be used as alternative initial investigation to CT in patients in which a CT may be contraindicated (for instance pregnancy). ${ }^{30}$

While in some parts of Europe US has gained acceptance as first line of investigation, this is in contrast to the general situation in most of the western world, where CT has become the preferred technique.

Computed Tomography-The gold standard cross-sectional imaging is multi-detector CT. It is minimally invasive and has sensitivity between $98 \%$ and specificity up to $99 \%$ for diagnosing AD. ${ }^{23,30,92,93}$ In the absence of contra-indications, intravenous contrast is typically used. In addition, oral or rectal water-soluble contrast can be added as adjuncts to enhance sensitivity and specificity depending on the clinical scenario. Multiple phases (including non-contrast and portal venous) are recorded.

Typical CT findings include the presence of diverticula, segmental, symmetrical wall thickening, hyper-enhancement and pericolic inflammation. In complicated disease, abscesses, localised or free intra-peritoneal gas, fistulisation or evidence of obstruction may be seen. ${ }^{94}$

Computed tomography has a role in confirming the diagnosis, but also predicting failure of non-operative management; extra-colonic contrast and distant free intraperitoneal gas are associated with the need for surgery. ${ }^{95,96}$ In order to enable more standardised reporting and prognostication, numerous classification systems have been proposed. The most recent is a modification of the previously described Hinchey classification of operative findings, ${ }^{61}$ in which AD is classified as 'Uncomplicated' or 'Complicated', and the latter subdivided from pericolic air through to diffuse fluid and distant free air depending on the degree of radiologic evidence of contamination (see Table 1). ${ }^{93}$ The authors proposed an algorithm for management based on a combination of the radiologic and clinical features of the individual case.

One of the concerns in the liberal use of CT, particularly in the increasing younger cohort of $\mathrm{AD}$ patients, is radiation dose. Walter et al have shown that lowering the dose of radiation by $50 \%$ may still provide sufficient diagnostic accuracy. ${ }^{97}$

Magnetic Resonance Imaging-Magnetic resonance imaging (MRI) is a radiation free alternative to $\mathrm{CT}$ (where $\mathrm{CT}$ is contraindicated) and provides good sensitivity and specificity (up to 94 and $92 \%$ respectively). ${ }^{88}$ It is less user-dependent than US and may be more sensitive and specific than US in patients with a higher body mass index or where another pathology is possible or suspected. ${ }^{30}$ 


\section{Criteria for Outpatient Management}

Historically acute diverticulitis has been managed in a hospital setting with intravenous antibiotics and 'gut rest'. Increasingly, this paradigm has been challenged and the value of antibiotics and hospitalisation questioned. The combination of an aging population and the increased prevalence of diverticular disease will drive an increase in presentations of $\mathrm{AD}$ and put pressure on limited in-patient systems. Hospital admission may be necessary for treatment or monitoring purposes however, early risk stratification can now be performed with a combination of clinical, laboratory and radiologic assessment and a decision made on the safety of out-patient management or the need for intervention.

\section{Summary of Evidence on Outpatient Management}

There has been increasing interest in the out-patient management of AD over the past 2 decades. The majority of data comes from prospective and retrospective cohort studies, however there is also one randomised controlled trial. ${ }^{98}$ The DIVER Trial was a prospective, multicentre randomised clinical trial (RCT) which compared hospitalisation and out-patient management in terms of treatment failure, the subsequent need for re-admission, quality of life and cost. ${ }^{99}$ While this trial showed no significant difference in treatment failure, readmission or emergency surgery requirement, it may have been underpowered as only one hundred and thirty-two patients were randomised (66 to each group). There was no significant difference seen in terms of quality of life assessment at 14 and 60 days but costs for the out-patient group were significantly lower at a third of the cost of in-patient treatment (savings of $€ 1124.70$ per patient). Moya et al also described savings of $€ 1600$ per patient managed as an outpatient and other studies have shown similar findings. ${ }^{100,101}$

This is generally consistent with the remaining literature. Pooled rates from a recent metaanalysis including 21 studies and 1781 patients show an overall failure rate of $4.3 \%$, where failure is defined as emergency admission to hospital within 60 days for AD. ${ }^{98}$ Subgroup analysis was not able to show an association between recurrent diverticulitis, comorbidities, peri-colic air, abscess or antibiotic type and treatment failure. However, previous studies have suggested that co-morbidity may predict readmission. ${ }^{102}$

Current consensus guidelines agree that there is a potential role for the out-patient management of $\mathrm{AD}$ although, the nature of what this entails is less clear. ${ }^{30,83,103-105} \mathrm{At}$ present, much of the mild disease is managed in the community by non-specialists, however a subset of those referred to hospitals will be able to be managed as out-patients once they are appropriately assessed.

\section{Typical Outpatient Regimen}

The majority of the studies on out-patient management establish the initial diagnosis based on clinical assessment and cross-sectional imaging with CT. Eligible patients were then given antibiotics in most protocols and started on a liquid only diet, which was gradually progressed over the following days. The most commonly prescribed antibiotics were Amoxicillin-Clavulanic acid or Ciprofloxacin and Metronidazole in penicillin-allergic patients. Duration of antibiotic therapy ranged from 7-14 days in those who gave antibiotics. $82,99,101,106,107$ 


\section{Non antibiotic treatment for diverticulitis}

Although this is covered in more detail elsewhere, this review would not be complete without mentioning the recent evidence against the routine use of antibiotics in uncomplicated AD. Administration of antibiotics has been standard of care for treatment of acute uncomplicated diverticulitis. However, recent data from two RCTs suggest antibiotics do not influence outcomes significantly and the role of antibiotics in otherwise healthy individuals is under question. A Swedish RCT of 623 patients found antibiotic treatment for acute uncomplicated diverticulitis neither accelerated recovery nor prevented complications or recurrence. ${ }^{108}$ A similar RCT from the Netherlands with 528 patients with CT proven $\mathrm{AD}$ found that observational treatment without antibiotics did not prolong recovery and can be considered appropriate in patients with uncomplicated diverticulitis. ${ }^{109}$ Both these trials were conducted in an inpatient setting. It is likely that we will see more patients with uncomplicated disease treated with observation in the future and this concept will need to be incorporated into outpatient management.

\section{The role of further investigation after an episode of acute diverticulitis treated as an outpatient}

Clinical follow up is important to ensure that the episode of AD has settled. Most recurrence is early and due to failure of the initial episode to settle. ${ }^{79}$ Ongoing follow up however is likely of little value, as there is little evidence of any active treatment reducing a further attack. It has been common practise for many patients to have a colonoscopy after an episode of diverticulitis to exclude an underlying colon cancer. Such investigation has a cost and risk (e.g. systemic effects of bowel perforation, colonoscopic perforation) associated with it. Recent evidence suggests that patients who have no pre excising symptoms (e.g. prior change in bowel habit, rectal bleeding and iron deficiency or anaemia ) and who have a good quality CT scan showing uncomplicated diverticulitis are at no greater risk of having a colon cancer that the general community. ${ }^{110,111}$

\section{Suggested Exclusion Criteria for Outpatient Management}

Based on the exclusion criteria used in the above studies, the following criteria for selecting the appropriate patients for out-patient management are proposed and summarised in the algorithm in Figure 1.

\section{Radiologic Diagnosis of Complicated disease}

The majority of studies exclude patients with radiologically complicated disease including the DIVER Trial and a number of the prospective cohort studies. ${ }^{99,101,106} \mathrm{~A}$ few studies included patients with small pericolic abscesses (defined as $<2 \mathrm{~cm}$ ) with acceptable treatment failure rates. ${ }^{112,113}$ However, given the conflicting evidence on the value of perforation in predicting treatment failure, it is proposed to exclude those with complicated disease. Better standardisation of radiologic classification may improve the ability to predict treatment failure based on imaging more consistently. ${ }^{93}$ 


\section{Immuno-compromised}

Immuno-compromise has consistently been used as an exclusion criterion in studies on $\mathrm{AD}$ due to the higher morbidity and mortality associated with $\mathrm{AD}$ in this population. ${ }^{114}$ It has been suggested that immune-compromise may result in a discrepancy between imaging and outcomes where free intra-peritoneal gas may not be associated with the typical pericolic inflammatory appearances that may therefore be missed or under-estimated

\section{Intolerant to Oral Intake}

In a survey of Australasian General Surgeons and New Zealand Colorectal Surgeons, intolerance to oral intake was considered an absolute indication for admission in 90 and $86 \%$ of respondents respectively. ${ }^{103}$

\section{Signs of Severe Sepsis}

Hypovolaemia was deemed an absolute indication for admission in 95\% NZ Colorectal Surgeon respondents, although simple observations such as a temperature over 38 degrees, a heart rate over 90 beats per minute or a respiratory rate over 20 per minute were less likely to warrant admission in isolation. 103

\section{Lack of Social Support}

This is an intuitive exclusion criterion that has been used in all of the studies. Inadequate social support is associated with a higher readmission rate in general surgery. ${ }^{115}$

\section{Comorbidity Score}

In some of the out-patient protocols, patients with co-morbidities were excluded from outpatient management, while in others (including DIVER) these patients were included regardless. ${ }^{98,99}$ Rueda included only those with American Anaesthesiology Association Score of I or II, ${ }^{102}$ while Alonso et al excluded patients with diabetes, heart failure, renal insufficiency or obstructive pulmonary disorder. ${ }^{106} \mathrm{It}$ is likely that it is the frail patients that are the common at risk group for failure of outpatient care.

A systematic review of the predictors of the severe AD identified a Charlson score of 3 or greater as an independent risk factor, ${ }^{116}$ a finding echoed in another similar meta-analysis of risk factors for complicated AD. ${ }^{117}$ However, in the radiologically diagnosed uncomplicated AD, the evidence is less apparent. ${ }^{98}$ Juang et al showed that a Charlson score of 3 or greater did not predict the need for procedural intervention, prolonged admission or readmission in a prospective study of patients with uncomplicated AD. ${ }^{118}$ Yoo et al showed no association between the presence of diabetes and need for surgery in their retrospective review of 117 patients with $\mathrm{AD} .{ }^{119}$ For these reasons comorbidity score is not considered an absolute contra-indication to the out-patient management of uncomplicated $\mathrm{AD}$, but clinical judgement should be used.

\section{Age Criteria}

Some studies included age criteria greater than 18 years, ${ }^{99}$ others less than 80102 or 90 years. ${ }^{101}$ This appears to be a relatively arbitrary parameter with no actual evidence to 
support it formally and frailty may be a more meaningful measure, given its association with poorer outcomes in acute general surgery. ${ }^{120,121}$

\section{Summary}

In summary, $\mathrm{AD}$ is a common presentation and is increasing in prevalence. Presentation may be suspected based on clinical and laboratory findings and is routinely investigated with cross sectional imaging (usually CT). Uncomplicated AD may be safely managed as an outpatient in carefully selected patients.

\section{Uncomplicated Diverticulitis}

Acute diverticulitis is a common clinical manifestation of colonic diverticula. Diverticular disease has been increasing in prevalence and it is estimated that lifetime risk of developing diverticulitis is around $20 \% .{ }^{30}$ The disease is more common in developed countries and has been associated with dietary habits with high intake of meats and refined sugars and low intake of fiber. ${ }^{36,122}$ The prevalence also increases with age from around $5 \%$ at age 40 to about $65 \%$ by the age of $80 .{ }^{123}$ Alcohol has been shown to increase risk of diverticulitis. ${ }^{124}$ Smoking is associated with increased risk of developing complications from diverticulitis. ${ }^{125}$ Obesity has been increasing in prevalence and studies continue to show association between obesity and risk of developing diverticulitis. ${ }^{52,126}$

Acute diverticulitis can be further sub classified into complicated and uncomplicated presentations. Uncomplicated diverticulitis is characterized by inflammation limited to colonic wall and surrounding tissue. Complicated diverticulitis is characterized by colonic inflammation and associated perforation, abscess formation, fistulation or stricture formation. The management of complicated and uncomplicated diverticular disease differs. Most patients with complicated diverticular disease are managed surgically in both elective and emergent settings. Uncomplicated diverticulitis however has been managed successfully non-operatively for decades. Recently there are new data which challenge conventional clinical practice in the management of uncomplicated diverticulitis. This includes use of antibiotics with an acute attack, role of routine endoscopy after uncomplicated diverticulitis and also role of elective surgery after uncomplicated disease. This article will review recent data relevant to these topics.

\section{Antibiotics in Uncomplicated Diverticulitis}

Since the advent of antibiotic therapy, antibiotics have been used routinely in the management of patients with acute diverticulitis both in inpatient and outpatient settings. $30,127,128$ Recently, investigators have proposed that acute uncomplicated diverticulitis may be an ongoing inflammatory rather than infectious process. ${ }^{129}$ This may suggest that the need for antibiotics in management of diverticulitis may be limited. Recent randomized controlled trials support this practice. The AVOD trial (Swedish acronym for "antibiotics in uncomplicated diverticulitis) was a Swedish multicenter, randomized controlled trial. ${ }^{108}$ The study included 623 patient with CT confirmed left-sided acute uncomplicated diverticulitis. The patient were divided into two groups. The first group were admitted and received antibiotics. The second group were admitted but only received IV fluids and no 
antibiotics. Patients with small pericolic abscesses were excluded. The rate of complications of colon perforation and abscess formation was comparable in both groups (No antibiotics vs Antibiotics: Perforation 3 vs 3\%: P-value 0.985, Abscess formation 3 vs 0\%: P-value 0.080). Seven patients required sigmoid colectomy either during index admission or during follow up in no antibiotics group vs 5 patients in antibiotics group (P-value 0.541). Risk of recurrent disease was $16.2 \%$ in no antibiotics group which was comparable to $15.8 \%$ in antibiotics group (P-value 0.881 ).

Another trial (DIABOLO) examining this subject was published in 2017. ${ }^{109}$ Patient were included from 22 different sites in Netherlands. All patient had CT proven, acute uncomplicated left sided diverticulitis. The primary outcome was recovery during a 6 months follow up period. Secondary outcomes included readmission rate, complicated diverticulitis within 6 months, ongoing and recurrent diverticulitis, need for sigmoid resection and mortality. Median time to recovery was 14 days in observation group compared to 12 days in antibiotics group. The negative secondary outcomes were higher in number in observation group compared to antibiotics group but this was not statistically significant (Complicated diverticulitis: $3.8 \%$ vs $2.6 \%$ [P-value 0.337], ongoing diverticulitis: $7.3 \%$ vs $4.1 \%$ [P-value 0.183 ], recurrent diverticulitis $3.4 \%$ vs $3 \%$ [P-value 0.494 ], sigmoid resections: $3.8 \%$ vs $2.3 \%$ [P-value 0.323 ], readmissions: $17.6 \%$ vs $12 \%$ [P-value 0.148 ], mortality: $1.1 \%$ vs $0.4 \%$ [P-value 0.432$]$ ]). However, the authors recognized the possibility of selection bias in the study and possibility of type II error. The study may not be powered to detect possible differences in the secondary outcomes.

In 2018, 2 year follow-up of DIABOLO study was published. ${ }^{130}$ There was no difference between groups in terms of recurrent diverticulitis (15.4\% in the observational group versus $14.9 \%$ in the antibiotic group: P-value 0.885$)$, risk of subsequent episode of complicated diverticulitis ( $4.8 \%$ vs $3.3 \%$ : P-value 0.403 ) and need for sigmoid resection (9\% vs $5 \%$ : Pvalue 0.085 ). Since the DIABOLO trial was not powered for secondary outcomes, these results need to be interpreted and applied in clinical practice with caution. Van Dijk et al looked at quality of life and persistent symptoms after management of uncomplicated diverticulitis with observation versus antibiotics. About one third of patients in each group had persistent symptoms at 1 or 2 years of follow up. No difference was seen in quality of life indices between the two groups. ${ }^{130}$ At this time it does appear that there are select patients who can be treated without the use of antibiotics however, further studies are needed to delineate groups that will benefit from this new approach to practice.

\section{Mesalamine, Rifaximin, and Probiotics}

As the role of microperforation in the pathophysiology of diverticular disease has come into question, there has been increased interest in the use of immunomodulatory agents in the management of diverticular disease. 5-ASA products and sulfasalazine alter DNA synthesis and cell cycle progression in lymphocytes. Because a low grade proinflammatory state is the proposed mechanism underlying chronic diverticular disease, a number of small trials have evaluated the effectiveness of mesalamine-like compounds. Earlier studies showed some promise of using these agents in management of diverticulitis patients. Trespi et al. demonstrated that patients treated with antibiotics and mesalamine had decreased 
symptomatology. ${ }^{131}$ Another study randomized patients with diverticulitis to a rifaxmin only arm versus a rifaximin/mesalamine arm. ${ }^{132}$ Patients in the rifaximin/mesalamine arm demonstrated significantly improved bowel habits. They also had less recurrent episodes and demonstrated lower symptom severity.

However more recent placebo controlled trials have failed to demonstrate clinical effectiveness of these products ${ }^{133,134}$. This was confirmed by recent meta-analysis by Urushidani et al. 1358 RCTs were reviewed in the study which demonstrated that 5-ASA products were not superior to controls in preventing recurrent diverticulitis (RR 0.86, 95\% CI 0.63-1.17) or decrease the incidence of adverse events (RR 0.97, 95\% CI 0.84-1.11).

The role of the gut microbiome is another topic of recent interest. Agents like rifaximin and probiotics are being investigated alone or in combination with fiber supplements. A metaanalysis from 2011 demonstrated that combination of rifaximin and fiber supplements were effective in reducing symptoms and preventing complications at 1 year. ${ }^{136}$ Regarding probiotics, a systematic review in 2016 showed that insufficient data is available currently to comment on role of probiotics in managing diverticular disease. ${ }^{137}$ More recent review however demonstrate that certain probiotics may have a role in all stages of diverticular disease and further research is needed to examine its role in management if diverticulitis patients. ${ }^{138}$

\section{Surgery for Uncomplicated Diverticulitis}

Acute uncomplicated diverticulitis is successfully managed conservatively in majority of patients. However the recurrent nature of disease, risk of potential chronic complications and effect of the disease on the quality of life continues to impact patients at multiple levels. The disease is also a recurrent healthcare burden. Recurrence after an episode of uncomplicated diverticulitis has been reported from $13-67 \%$ in studies with variable follow up periods. $53,56,139-141$ Review of literature shows that clinical practice guidelines have gone through multiples changes regarding appropriate surgical managements of these patients.

Clinical practice guidelines in 2000 recommended surgery after two attacks of uncomplicated diverticulitis. ${ }^{142}$ In 2006, it was suggested that number of attacks in not necessarily the most important factor in deciding need for surgery and may be younger patients should be considered for surgery due to their higher cumulative risk for recurrent disease. ${ }^{128}$ In 2014 individualized approach was recommended for surgery after recovery from uncomplicated acute diverticulitis. ${ }^{30}$

Investigators have been interested in identifying appropriate groups of patients to intervene on surgically and the optimal timing of a surgical approach. A single institution retrospective review by Hall et al showed the family history of diverticulitis, length of affected colon $>5$ $\mathrm{cm}$ and presence of retroperitoneal abscess at index presentation were associated with risk of recurrent disease. ${ }^{56}$ Other authors have demonstrated that the risk of recurrence increases with each episode of diverticulitis. Bharucha et al showed the rate of recurrence after first and second episodes of diverticulitis is $22 \%$ and $55 \%$ at ten years respectively. ${ }^{53}$ At the same time, risk of subsequent complicated disease after an episode of uncomplicated diverticulitis is less than $5 \% .{ }^{56,108,109}$ Chapman et al showed similar findings where patient 
with $>2$ episodes of diverticulitis were not at higher risk of complications compared to patients with 1 or 2 episodes of diverticulitis challenging the idea of offering elective resection after certain number of episodes of uncomplicated diverticulitis. ${ }^{21}$ On the other hand, complicated diverticular disease has a higher recurrence rate and recurrent episodes are usually more severe compared to the index presentation. ${ }^{143}$ Hence the decision to recommend surgery should not depend on number of episodes of recurrent diverticulitis.

Another area of recent research interest is to compare quality of life in patients receiving conservative management with those undergoing elective sigmoidectomy. One such trial is the DIRECT trial (Surgery versus conservative management for recurrent and ongoing left sided diverticulitis). This study compared surgery versus conservative management for recurrent and ongoing left-sided diverticulitis. ${ }^{144}$ This is a multicenter randomized controlled trial from Netherlands. Patients aged 18-75 with three recurrent episodes or ongoing symptoms of diverticulitis were randomly assigned to either conservative management or surgical intervention groups. The primary end point of the study was quality of life measured by the Gastrointestinal quality of life index (GIQLI). Secondary end points included additional quality of life assessments and also risk of morbidity and mortality associated with each approach. Mean GIQLI score in surgical group was higher at 6 months compared to conservative management group (P-value 0.0001 ). Secondary outcomes were also found to be better in surgical group. $23 \%$ of patients in conservative group ultimately required surgical intervention. This trial concluded that surgical intervention, despite its risks, lead to better quality of life compared to patients managed conservatively. Another ongoing trial is Comparison of surgery and medicine on the impact of diverticulitis (COSMID) trail. This is a large scale, randomized controlled trail that will compare outcomes of surgery with best medical management for quality of life limiting diverticulitis. The study will report both patient related and clinical outcomes.

\section{Young patients}

Several authors have proposed that patients younger than 40 to 50 years of age present with a move virulent form of diverticulitis. ${ }^{145,146}$ Historical recommendations have advocated sigmoid resection for young patients after one well established attack of diverticulitis however, this dictum has been called into question by recent evidence. Data suggest that young age is not necessarily associated with worse clinical outcomes. In a study by Guzzo et al., risk of requiring surgery on initial presentation was similar in patients younger than 50 compared with older population group. ${ }^{26}$ One patient out of 196 young patients ( $<50$ years) had a free perforation after medical management of diverticulitis. Review of large administrative dataset suggested that young patients may indeed have a higher risk of recurrence (27\%) but have low and comparable rates of emergency surgical intervention (7.5\% vs $5 \%$ ). ${ }^{20}$ Another retrospective study showed that young patients with CT diagnosed diverticulitis respond well to medical management and have low subsequent risk of emergency surgery and need for colostomy. ${ }^{147}$ Given the current level of evidence there is no clear mandate to treat patients with young patients with diverticulitis differently than other age groups. 
Clinicians agree now that decision of surgery for uncomplicated diverticulitis is not dependent of factors like age of the patient or number of previous episodes. Rather the decision should be individualized to each patient. Imaging from prior episodes should be reviewed to ensure that prior episodes were properly staged. Attention should be paid to factors like how severe were the prior episodes, how long the antibiotics were needed before resolution of symptoms and whether patient had full resolution of symptoms or still has smoldering symptomatology. The decisions should be based on evaluating risk of surgery with patient's risk of recurrent disease and persistent symptoms affecting quality of life. ${ }^{148}$

\section{Surgical Technique}

As role of surgical intervention continues to evolve in management of acute diverticulitis, the role of standardized surgical technique to compare outcomes is becoming equally significant. Laparoscopic surgery is becoming increasingly prevalent in clinical practice. The Sigma trial was a prospective, double blinded, multicenter, randomized controlled trial. ${ }^{149} \mathrm{It}$ compared 104 patients with diverticular disease undergoing either laparoscopic or open surgery. The rate of conversion was $19.4 \%$ in the laparoscopic group. The short term outcomes showed $15.4 \%$ reduction in complication rates, lower post-operative pain, improved quality of life and shorter hospitalization rate in the laparoscopic group of patients. Operating time was longer for laparoscopic surgery. Follow up study of the trial showed that this difference in outcomes between open and laparoscopic approaches decreases over time and at 6 months there was no significant difference between the two groups in terms of mortality and late complications like incisional hernias, bowel obstructions, abscess formation and enterocutaneous fistulas. ${ }^{150}$

Authors have also compared the level of proximal and distal transection during the primary operation as it relates to disease recurrence. The purpose of surgery is to remove disease segment of colon and to reestablish intestinal continuity and not to remove all diverticula bearing colon. Proximal resection margin is decided intra-operatively based on anatomy. This is up to area of proximal non inflamed, non-hypertrophied and compliant colon. However since diverticulum is an area of weakening in the colon, care should be taken not to incorporate them in the anastomosis. Distal margin is of great importance in decreasing incidence of recurrent disease. Report from Mayo clinic compared 501 patients undergoing sigmoid colectomy with either colo-sigmoid or colo-rectal anastomosis. Recurrent disease was $12.5 \%$ in colo-sigmoid anastomosis group compared to $6.7 \%$ in colo-rectal anastomosis group. ${ }^{151}$ Similar findings were reported by Thaler et al who demonstrated a 4 times higher risk of recurrence if distal sigmoid colon was used for anastomosis compared to upper rectum. 152

Anastomotic leak after colectomy has significant morbidity and mortality. High ligation of inferior mesenteric artery (IMA) is considered important step in oncologic resection for sigmoid and rectal cancers. However this is not considered mandatory in sigmoid resections for diverticular disease. Studies have examined the role of inferior mesenteric artery (IMA) preservation on the rate of anastomotic leak. A retrospective review of 130 patients by Lehmann et al showed no increase in leak rate when IMA was sacrificed. ${ }^{153}$ Similar results were reported in a meta-analysis by Cirocchi et al. No statistically significant difference was 
found in rate of anastomotic leak when IMA was preserved or sacrificed during sigmoid colectomy. ${ }^{154}$ This finding has been confirmed in recent studies and meta-analysis. Recent studies are in agreement with these earlier studies. A recent study looked at role of IMA preservation in regards to functional outcomes after sigmoid colectomy. The idea was that more proximal vascular transection will lead to colonic denervation and worse functional outcomes. The study results confirmed that in 54 out 107 patients in whom IMA was preserved, defecatory function and overall quality of life indices were better compared to those in whom IMA was sacrificed. ${ }^{155}$ This study highlights the possible role of IMA preservation in improving functional outcomes after diverticular surgery. Overall, currently there is not enough evidence to support routine preservation of IMA in surgical management of diverticular disease.

Tension at colorectal anastomosis is an important technical factor with risk of higher rates of anastomotic strictures and leaks. Splenic flexure mobilization (SFM) is one of the maneuvers undertaken to decrease tension at anastomosis. Cadaveric study showed an additional colon length of $10.7 \mathrm{~cm}$ achieved with SFM. This is increased to $28.3 \mathrm{~cm}$ with distal transverse colon mobilization. ${ }^{156}$ However there is no consensus regarding need for routine SFM for left sided colectomy. Studies in patients with rectal cancers have shown that routine SFM is associated with longer operative time with no difference in anastomotic complications, local recurrence and postoperative morbidity and mortality. ${ }^{157,158}$ Recent review on SFM in colon resection for diverticulitis also showed no benefits of routine mobilization and showed a trend towards more minor post-operative complications. ${ }^{159}$ Hence while there has been an increase in the use of SFM in patients undergoing laparoscopic surgery for diverticulitis, there appears to be no benefit with routine use of this approach. The decision to perform SFM should be individualized based on intra-operative findings.

Leak test should be routinely performed after colectomy for sigmoid diverticulitis. This will identify suboptimal anastomosis and allow for recreation or repair of anastomosis and guide decision for need to divert proximally. Ricciardi et al showed that rate of clinical leaks was higher in patients with positive intra-operative leak test. He also showed that suture repair after positive leak test was associated with higher postoperative leak rate compared with recreation of anastomosis or proximal diversion. 160

\section{Summary}

The management of uncomplicated diverticulitis is changing. Use of antibiotics has been questioned. It appears that antibiotic use can be avoided in select groups of patients. Surgical intervention appears to improve patient's quality of life. The decision to proceed with surgery is recommended in an individualized manner. Meticulous adherence to standard surgical techniques will help to improve outcomes for patients with this disease.

\section{Complicated Diverticular Disease}

Complicated diverticulitis is defined as diverticulitis associated with localized or generalized perforation, localized or distant abscess, fistula, stricture or obstruction. The Hinchey classification system has been used to compare and stratify patients with complicated 
diverticulitis. Hinchey Stage I diverticulitis is associated with pericolic abscess, Stage II with more distant abscess like pelvic or retroperitoneal abscess, Stage III with purulent diverticulitis and Stage IV patients include those with feculent peritonitis. Modified Hinchey Classification was proposed by Warsavary et al., but the original Hinchey Classification is still the most used clinical classification in practice. (Table 1) Another modification based on CT findings was proposed as well. ${ }^{161}$ (Figure 2)

\section{Diverticular abscess}

Diverticular abscess can occur in about $10-15 \%$ of cases. ${ }^{162}$ The incidence of diverticular abscess has increased over the last decade likely due to increased utilization of crosssectional imaging in the last $10-20$ years. These cases can present either locally around the sigmoid colon (pericolic, mesocolic or pelvic) or at more distant sites in the form of subhepatic abscess. Clinical symptomatology depends on the location of the abscess and the extent of inflammation and peritoneal contamination. Radiological cross-sectional imaging with CT scan is the most effective way to diagnose diverticular abscess. Clinical exam along with the information from cross sectional imaging would help with formulating treatment plans for patients with diverticular abscess. The size of the abscess, its location, presence of loculation and the extent of the peritoneal contamination or presence of peritonitis has been used to decide the treatment algorithm for the individual patient.

\section{Perforated Diverticulitis}

Complicated diverticulitis presenting as free perforation occur in about $1 \%$ of patients presenting with diverticulitis. Patients may manifest with symptoms of generalized or localized peritonitis at the time of presentation in the emergency room or during the first 24 - 48 hours after admission to the hospital. Free perforation usually occurs on the first attack of diverticulitis and is not usually seen in patients who have had multiple attacks of diverticulitis. Clinical presentation may include signs of localized peritonitis or generalized peritonitis. Generalized peritonitis may present with hemodynamic instability. The presence of feculent or purulent peritonitis, the extent of contamination and hemodynamic status and associated comorbidity decide the treatment options for the patients. ${ }^{162}$

\section{Diverticular Fistulas}

Fistulas complicating diverticular disease occurs in about $2 \%$ of the patients. The localized inflammatory process associated with the colon decompressing into the adjacent viscera leads to the formation of the fistulous process. Fistulous process associated with diverticular disease is usually a chronic presentation and symptomatology associated with the fistula is related to the type of fistula with usually fewer abdominal signs and symptoms. Colo cutaneous fistula could be a sequel of peri colonic abscess. Colo vesical, Colovaginal, coelenteric, Colo colonic, Colo uterine or other common fistulation process. Colo appendiceal, Colo gastric, Colo venous, Colo perineal, Colo perianal, and Colo ureteral are other uncommon internal / external presentations associated with complicated acute diverticulitis. ${ }^{163-166}$ 


\section{Colovesical fistula}

Colovesical fistula is the most common type of (65\%) of internal fistulation process associated with complicated diverticulitis. It is usually a mature fistula and occurs usually in association with chronic diverticulitis. Colo vesical fistulas are more common in men with a male-to-female ratio of 2 to 3:1 likely due to intervening broad ligament and uterus between the urinary bladder and sigmoid colon. More than $50 \%$ of women with colovesical fistula have had previous hysterectomy which supports the above theory. The mean age at the time of presentation is between 55 and 75 years. ${ }^{166-168}$ Patients with Colo vesicular fistula typically present with pneumaturia, fecaluria, dysuria and recurrent urinary tract infections. Most patients have urinary tract infections and dysuria. Pneumaturia and fecaluria are pathognomonic symptoms of colovesical fistula and seen in $75 \%$ and $50 \%$ only respectively. Poppy seed test is diagnostic in $94.6 \%$ patients, CT in $60.0 \%$, MRI in $60.0 \%$, retrograde colonic enema in $35.7 \%$, Cystogram in $16.7 \%$, Cystoscopy in $10.2 \%$, and Colonoscopy in $10.2 \%$ patients. ${ }^{164}$ (Figures 3)

Recurrent urinary tract infections could be presenting symptoms without any associated prior episodes of diverticulitis. CT scan of the pelvis is helpful in the diagnosis with inflammation usually localized to the dome of the bladder, with or without air within the lumen of the bladder. Cystoscopy when performed shows inflammation at the dome of the bladder and on occasion, food material like vegetable matter could be seen in the urine.

\section{Colovaginal and Colouterine fistula}

Colovaginal fistulas are the most common diverticular fistula to the female genital organ and most commonly occur in older women with history of prior hysterectomy. History of diverticulitis preceding colovesical fistula diagnosis is noted in only a third of the patients. Presenting symptoms include vaginal discharge (95\%), passage of air or stool through the vagina. Abdominal pain is reported in only $50 \%$ of patients. ${ }^{166,169}$ These patients usually present to their primary physicians or were seen by their gynecologist. Cross sectional imaging would help with the diagnosis with the inflammatory process involving the colon over the vaginal cuff. (Figure 4) An opening seen at the apex of the vagina is seen in 30$87 \%$ patients, and most commonly on the left side. 169,170

Colouterine fistulas are rare phenomenon and literature about them is limited to case reports only. It is more likely that colouterine fistulas are likely from non-diverticulitis related etiology like secondary to obstetric trauma, pelvic malignancy and effects of radiation therapy.

\section{Colocutaneous fistula}

Colo cutaneous fistula is uncommon and result from inadvertent incision and drainage of abdominal wall abscess associated with acute complicated diverticulitis associated with parietal wall abscess. (Figures 5\&6) Patients who have had percutaneous drainage of diverticular abscess may also present with Colo cutaneous fistula prior to their elective resection. Patients who have had sigmoid colectomy for diverticular disease with anastomosis to the distal sigmoid rather than proximal rectum are at higher risk for 
Colocutaneous fistula with recurrent diverticulitis involving the distal sigmoid colon.

Patients with Colocutaneous fistula may also have unsuspected Crohn's disease. ${ }^{171}$

\section{Coloenteric Fistula}

Coloenteric fistulas are uncommon fistulas and comprise between 3 to $7 \%$ of all diverticular fistulas. ${ }^{166,172}$ It is usually between a redundant sigmoid colon and terminal ileum and less commonly with jejunal loops. Presenting symptoms include diarrhea, which presents as sudden development of severe watery diarrhea. Abdominal pain and weight loss secondary to diarrhea are other common presenting symptoms. CT scan may help with the diagnosis and malignant fistula should be excluded with colonoscopy. ${ }^{173}$ Coloenteric fistula may be accompanied by distal sigmoid stricture as well. ${ }^{174}$

\section{Colonic Stricture and obstruction}

Colonic stricture and obstruction can have either an acute or may have a more chronic clinical presentation. (Figure 5) Complicated diverticulitis with significant inflammation can present with an inflammatory stricture or phlegmon can present with partial or complete large bowel obstruction. These patients would require more urgent surgical intervention.

Patients who have had multiple episodes of diverticulitis over many years treated conservatively may present with chronic left lower quadrant cramping abdominal pain. Barium enema or flexible sigmoidoscopy may demonstrate the obstruction and usually mandate surgery to address obstruction. Colon cancer and ischemic strictures are important differential diagnosis when large bowel obstruction is the presentation.

\section{Diverticular hemorrhage}

Diverticular bleeding accounts for nearly 200,000 hospital admissions in the United States annually and occurs in 3-5\% of patients with diverticulosis. It usually presents as painless, intermittent and large volume lower GI bleeding. ${ }^{174}$ Diverticular bleeding is the most common cause of lower gastrointestinal bleeding, accounting for 20.8 to $41.6 \%$ of cases of lower gastrointestinal bleeding. ${ }^{175}$

Advanced age, NSAID, anticoagulant as well as antiplatelet use are the most common risk factors for the incidence diverticular bleed. ${ }^{176}$ The nature and color of the bleeding depends on the intensity of the bleeding, with right sided diverticular bleeding presenting more like Malena and left sided diverticular bleeding present as bright red bleeding per rectum. In stable patients, colonoscopy remains the gold standard in diagnosis and treatment of diverticular bleeding. Stigmata of bleeding, including active hemorrhage, visible vessel, adherent clot to the diverticulum are diagnostic of diverticular hemorrhage. ${ }^{177}$ Patient factors, such as the use of oral anticoagulants or antiplatelet agents, hemorrhagic shock on presentation and major stigmata of bleeding in colonoscopy are risk factors for rebleeding. 178,179 Patients who remain hemodynamically unstable after initial resuscitation and are unable to tolerate a bowel preparation for colonoscopy, should get a CT angiography, followed by embolization if an active bleeding source has been identified. ${ }^{180}$ 


\section{Immunocompromised Patients}

Patients with immunocompromised status and acute diverticulitis are a special category of patients and managed differently. This group of patients include those with immunocompromised disease like untreated HIV, organ transplant recipients on immunosuppressants, systemic steroids, diabetes mellitus, renal failure, cirrhosis and underlying malignancy patients on chemotherapy or radiation therapy. Immunocompromised patients are more likely to present with free perforation due to inability to mount appropriate inflammatory response to the acute events. These patients are likely to require emergency surgery with resultant increased risk of perioperative complications. ${ }^{181}$

Role of Colonoscopy-After an episode of acute diverticulitis, routine colonoscopy has been recommended by ACPBGI and ASCRS. Colonoscopy is advocated to exclude the presence of colorectal cancer or presence of alternative diagnosis like ischemic colitis or inflammatory bowel disease for the clinical presentation. Endoscopic evaluation of the colon is normally delayed by about 6 weeks from the acute episode to reduce the risk associated with colonoscopy. The World Society of Emergency Surgery recommends early colonoscopy (4-6 weeks) after an acute episode after a diverticular abscess treated conservatively (Recommendation 1C) but recommend only age appropriate colon cancer screening for patients with uncomplicated diverticulitis treated conservatively with appropriate response.

The need for early colonoscopy is based on the premise that colon cancer can mimic diverticulitis, and colon cancer or advanced adenoma may be associated with the current episode of diverticulitis. But this historical clinical concern must be viewed taking into consideration the advances in cross sectional imaging technology and also the diagnostic criteria used to make the diagnosis of acute diverticulitis. With the use of CT criteria of inflamed diverticula, pericolic fat stranding, fluid at mesentery and preserved bowel enhancement pattern, the overall diagnostic accuracy of CT scan for acute diverticulitis has been shown to be approximately $99 \%$. ${ }^{182}$ Studies have shown CT findings of diverticulitis and colon cancer is so high that an unequivocal diagnosis can be made with $100 \%$ accuracy for both diseases. ${ }^{183}$

Colonoscopy, although routinely very safe generally, is also associated with serious complications, including colorectal perforation, and has a higher failure rate of cecal intubation in the background of acute diverticulitis. ${ }^{184}$

Multiple retrospective studies and meta-analysis have evaluated the incidence of colon cancer diagnosed during a follow up routine colonoscopy after an episode of acute diverticulitis. Meta-analysis by Sai et al. showed a higher prevalence $(2.1 \%$ vs $0.68 \%$ ) of colon cancer in patients with recent diverticulitis compared with general population. But this has been attributed more due to selection bias and inappropriate design of the study to address this question, than true incidence. The Meta-analysis using SEER database to compare the results and found slightly higher rate of calculated prevalence of colorectal cancer in the diverticulitis group compared with population of comparable age. ${ }^{185}$

Multiple other studies have also been very critical of routine consideration of colonoscopy after an episode of acute uncomplicated diverticulitis. A prospective study by Lahat et al. 
evaluated the clinical utility of routine colonoscopy and showed that colonoscopy performed immediately after an episode of acute diverticulitis added no clinical value, except for those with a more protracted course of acute diverticulitis. Their study also evaluated the utility of waiting 6 weeks after an acute episode of diverticulitis. The authors performed colonoscopy after medical treatment of acute diverticulitis in the same admission prior to discharge.

Colonoscopy was performed 4-12 days after admission (median 5.8 days). Complete colonoscopy was achieved in $82 \%$ of patients, with colonic stricture at the site of diverticulitis, malignant obstruction, and poor colonic perforation being the reason for the incomplete colonoscopy in $18 \%$ of patients. The authors reported a higher risk (16.6\%) of colonic perforation with early colonoscopy in patients whose index CT scan showed air around the colon associated with diverticulitis. ${ }^{186}$ A retrospective study by Lam et al. showed that in fact the prevalence of colon cancer in patients with diverticulitis is lower than the lifetime risk of colon cancer and diverticulitis is not a risk factor for development of colorectal cancer and adenomas. ${ }^{187}$ Another retrospective study by Lau et al. evaluated the results of routine colonoscopy after acute diverticulitis in 319 patients, and reported $26 \%$ incidence of polyps, $2.8 \%$ incidence of colorectal cancers. The odds of a diagnosis of colorectal cancer were 6.7 times (95\% CI 2.4-18.7) in patients with an abscess reported on the initial CT, 4 times (95\% CI 1.1-14.9) in patients with local perforation, and 18 times (95\% CI 5.1-63.7) in patients with fistula compared with patients with uncomplicated diverticulitis. The authors recommended routine colonoscopy after left sided diverticulitis in patients who have not had recent colonoscopy. The rate of associated cancer is higher in patients with associated abscess, local perforation or fistula. ${ }^{188}$ Multiple other studies showed poor yield with routine colonoscopy after acute uncomplicated diverticulitis but did recommend interval colonoscopy after complicated diverticulitis. Similar results are also reported by multiple other studies. ${ }^{111,185,189}$

But, another study from the United Kingdom reported $2.1 \%$ of colon cancer with follow up colonoscopy after acute diverticulitis, compared with $1.6 \%$ in asymptomatic screened population. The authors of this study were skeptical about missing colon cancer despite good cross-sectional imaging and recommended repeat CT imaging or CT colonography, should the patients be not offered or not elected to have a follow up colonoscopy. 190

A recent meta-analysis by Rottier et al, included nine studies, and included a total number of 2,490 patients with uncomplicated diverticulitis. Subsequent colonoscopy after uncomplicated diverticulitis was performed in 1,468 patients (59\%). Seventeen patients were diagnosed with CRC, with a prevalence of $1.16 \%$ (95\% confidence interval 0.72-1.9 $\%$ for CRC). Hyperplastic polyps were seen in 156 patients (10.6\%), low-grade adenoma in 90 patients $(6.1 \%)$, and advanced adenoma was reported in 32 patients $(2.2 \%)$. The results of this review demonstrated that unless colonoscopy is indicated for screening needs in individuals aged 50 years and older, routine colonoscopy in the absence of other clinical signs of CRC is not required in patients following an episode of acute uncomplicated diverticulitis. ${ }^{191}$ Similar results were also reported from another systematic review and meta-analysis evaluating the role of routine colonic evaluation after radiologically confirmed acute diverticulitis was published in 2014. ${ }^{110}$ This study included eleven studies from 7 countries, with 1970 patients, and reported a pooled proportional estimate of colorectal malignancy was $1.6 \%$ (95\% confidence interval $[\mathrm{CI}], 0.9 \%-2.8 \%)$. There was significant 
difference between uncomplicated and complicated diverticulitis with proportional estimate of risk $0.7 \%$; CI, $0.3 \%-1.4 \%$ ), after uncomplicated diverticulitis and proportion estimate of risk 10.8\%; CI, 5.2\%-21.0\%) after complicated diverticulitis. These authors as well reported forgoing colonoscopy after routine uncomplicated diverticulitis and routine reported significant risk of colon cancer during follow up colonoscopy after copmlciated diverticulitis. Another systematic review published in 2014 by de Vries et al. also opined higher confidence in definitive diagnosis of acute diverticulitis without need for routine diagnostic colonoscopy. Patients with other indications for colonoscopy like age more than 50 without recent prior colonoscopy, rectal bleeding, complicated diverticulitis should be considered to have higher risk for colorectal cancer and should be offered follow up colonoscopy. ${ }^{192}$ Another metanalysis by Assad et al. also showed similar results. Their metanalysis included studies from 4 different countries (Netherlands, France, South Korea and United Kingdom) with 3082 cumulative patients, with 1022 in the diverticulitis group and 3437 in the screening group. It did not show any difference in the risk of polyps, advanced and non-advanced adenomas and cancer between the two groups. ${ }^{193}$

The concern about different recommendation for patients based on their age ( $<50$ vs. $>50$ years of age) based on increasing risk factors for colon cancer with advancing age has also been evaluated in various studies. Studies by Lecleire et al. showed lower utility of routine colonoscopy in less than 50-year-old patients after an uncomplicated episode of diverticulitis compared with age and sex-matched average-risk population undergoing a screening colonoscopy. ${ }^{194}$ Similarly, the role of routine colonoscopy after acute right sided diverticulitis in younger Asian men also showed no increased benefit with routine follow up colonoscopy. ${ }^{195}$ The recently published SAGES and EAES 2018 consensus conference on acute diverticulitis management do not recommended routine colonoscopy in the absence of high risks features, after an episode of uncomplicated acute diverticulitis. ${ }^{196}$

The authors recommend the following from the available evidence:

1. Routine colonoscopy after an episode of acute diverticulitis characterized by typical clinical symptoms and radiological imaging, is shown to have low yield.

2. The outcomes from routine colonoscopy after diverticulitis in different age group ( $<50$ and $>50$ years of age) are not very different. Also, the results have been same for both right side and left sided diverticulitis.

3. Studies have shown more yield (increased rate of advanced adenomas) from colonoscopy after an acute complicated diverticulitis (Hinchey's classification III $\&$ IV). Authors recommend routine colonoscopy prior to elective surgery for diverticular disease, as these clinical scenarios are usually secondary to complex or complicated diverticular disease process.

4. Colonoscopy should also be routinely performed after complicated diverticulitis cases, when the clinical presentation is atypical or if there are any diagnostic ambiguity, or patient has other indications for colonoscopy like rectal bleeding or is above 50 years of age without recent colonoscopy. 


\section{Resection after Diverticular Abscess Management - is it necessary?}

Approximately $10-20 \%$ of patients with acute diverticulitis will present with complicated diverticulitis in the form of an associated abscess and this presentation was historically an indication for surgical resection. \{Hall, $2010 \# 659$ \} Controversy now exists on the optimal treatment of diverticulitis with abscess and specifically, those who resolve with initial nonoperative management of their abscess.

Improvements in both medical and radiographic management and technology have greatly reduced the rates of resection for those who present with a diverticular abscess at the index presentation. Kumar and colleagues demonstrated that non-operative management with either antibiotics alone or antibiotics plus percutaneous drainage was successful in over 90 percent of patients presenting with abscess thus avoiding an urgent operation in an inflamed, scarred and hostile environment likely to require an ostomy. \{Kumar, 2006 \#660\} Risk factors for those requiring surgery were size of an abscess $25 \mathrm{~cm}$ or pelvic location. \{Ambrosetti, 2005 \#661;Kumar, 2006 \#660\} Accordingly, in 2014 the American Society of Colon and Rectal Surgeons released practice parameters on the management of diverticulitis stating that, 'Following successful medical treatment of mesocolic abscesses of $25 \mathrm{~cm}$ or pelvic abscesses with or without percutaneous drainage, elective colectomy should typically be advised'. \{Feingold, 2014 \#219\} However, elective resection after successful abscess treatment without persistent fistula is debated due to emerging knowledge regarding the natural history of diverticulitis such that it is not a progressive disease and the severity of recurrent disease mirrors that of the index presentation. \{Strate, 2012 \#569\} This begs the question that if diverticular disease is not a progressive disease, do we really need to operate after successful treatment of a diverticular abscess or asked in another way, will operating electively after successful abscess treatment decrease subsequent episodes and/or emergency surgery?

In 2013, Gaertner et al published their 5-year data of over 200 patients who underwent initial non-operative treatment for diverticular abscess with either antibiotics alone or combined with percutaneous drainage. \{Gaertner, 2013 \#662\} In their study 32 patients (15\%) did not undergo subsequent elective colectomy after successful non-operative treatment of their abscess and the overall risk of recurrence was $31 \%$ with a recurrence free survival rate of $58 \%$ at 7.4 years. The biggest risk factor for recurrence was an abscess $25 \mathrm{~cm}$, regardless of location, and if they did recur, they presented in a similar or more mild fashion than the index episode, keeping with what we know about the non-progressive nature of the disease. Additionally, none of the patients with recurrence required operative intervention suggesting that non-operative management of a diverticular abscess without subsequent elective colectomy is acceptable and safe. This data is consistent with other published reports where over 50\% of patients who had successful treatment of their mesocolic abscess at index presentation were able to avoid subsequent elective operative intervention at a mean followup of 43 months. \{Ambrosetti, $2005 \# 661\}$ Conversely, data from Kaiser et al suggest that elective colectomy after nonoperative treatment of a diverticular abscess is still recommended as $42 \%$ of patients who did not undergo elective colectomy after index presentation had a recurrence and this was associated with an increased probability of colostomy creation (40\%). \{Kaiser, $2005 \# 17\}$ 
However, these studies fall victim to significant limitations as most are small, single institution, retrospective in nature with limited follow-up and most importantly, have significant patient selection bias. In nearly all series, the patients who did not undergo elective colectomy after successful non-operative management of their diverticular abscess were considered poor operative candidates due to significant comorbidities such as immunosuppressed or transplant status, or severe cardiopulmonary disease. Interpreted another way, this data suggests that even in the highest risk patient population, the only risk of not undergoing elective colectomy after abscess is the risk of recurrence and operation at a later date. This implies that a healthier patient population might have better results. To overcome this selection bias, Garfinkle and colleagues published data on 73 patients who were overall healthy and who presented with diverticular abscess treated with non-operative intent both at index presentation and longterm. \{Garfinkle, 2016 \#663\} Due to limited operating room availability in their healthcare system, non-operative long-term management has been their standard approach for those who were asymptomatic after successful treatment of a diverticular abscess regardless of health status. Their data show that after successful treatment of the initial episode, approximately $30 \%$ experienced a recurrence at a mean of 23 months of which only 2 required urgent operative intervention. Additionally, $87 \%$ of patients avoided an operation all together despite one-third having a recurrence requiring hospitalization. They conclude that long-term non-operative intent is safe with a low failure rate and low rate of urgent operative intervention. A meta-analysis by Lamb et al attempted to summarize the data and showed that $38 \%$ of those waiting for their elective resection and $17 \%$ of patients not considered for surgery experienced a recurrence. \{Lamb, 2014 \#664\} Only 28\% of patients avoided surgery and/or a recurrence all together suggesting that while elective surgery is not mandatory, routine non-operative intent is associated with high recurrence and need for an operation.

The optimal long-term strategy for patients who undergo successful non-operative management of their diverticular abscess remains controversial. There are clearly patients who would do well with an elective colectomy and a subset who could avoid an operation all together however, the challenge is appropriate risk-stratification and patient selection. Therefore, until more high quality, prospective data becomes available, the decision for surgery in asymptomatic patients after non-operative treatment for diverticulitis with abscess should be individualized.

\section{Complicated patient with diverticulitis: what is the medical and operative plan?}

Introduction

As with treatment of any other disease, success or failure of treatment for diverticulitis is influenced greatly by both patient factors as well as surgical management. A number of patient factors can increase risk for complications and death. Many of these are modifiable and the goal is to address and improve these patient factors prior to surgery. Risk modification can occur in the both the short and long term. The benefits of improving the preoperative health of patients is gaining in popularity (https://www.facs.org/qualityprograms/strong-for-surgery). Additionally, optimization of both surgical and peri-operative 
care has been shown to improve patient outcomes. Collectively, these interventions seek to mitigate the burden of co-morbidities and increase the chances of successful medical and surgical treatment of diverticulitis.

\section{Immunosuppression}

Immunosuppression can be the end results of a number of different disease processes, including transplant immunosuppression, long-term glucocorticoid therapy, or from chronic medical conditions such as renal failure, diabetes, collagen-vascular disorders such as lupus, or malnutrition. Patients with human immunodeficiency virus (HIV) infection or acquired immunodeficiency syndrome (AIDS) are also to be considered immunosuppressed. But as the mechanism of immunosuppression differs, they are not normally included under the classic definition of immunosuppression for the purpose of this paper.

Immunosuppression has long been identified as a complicating factor in the treatment of diverticulitis. Contrasted to the general population, patients who are immunosuppressed are more likely to develop acute diverticulitis, more likely to require emergency surgery if they developed diverticulitis (10-25\% versus $40 \%$ ), and have increased mortality if they undergo emergency surgery for diverticulitis ( $<5 \%$ versus $30 \%) .{ }^{197-199}$

Because of the greatly increased risk of emergency surgery, most surgeons should maintain a low threshold to recommend elective resection after a single episode of diverticulitis in an immunosuppressed patients. This rational also extends to patients being considered for transplantation given the need for long term immunosuppression. However, this should only be considered in the instance where the underlying disease process is controlled (ie a end stage renal disease patient on dialysis).

Long term corticosteroid use has been shown to increase the risk of anastomotic leak. ${ }^{200}$ Therefore, anastomoses should be protected by a diverting stoma or a Hartmann procedure should be considered to avoid the morbidity of an anastomotic leak.

For patient with HIV or AIDS, there is insufficient evidence to recommend a more aggressive elective colectomy. However, optimization of antiretroviral therapy (ART) should be employed prior to any elective surgical resection. ${ }^{201}$

\section{Obesity}

Worldwide, the overweight/obese population has been steadily increasing. According to the WHO, with 1.3 billion overweight $(25<$ body mass index [BMI] < 30) people and 600 million obese (BMI > 30) people in the world, the obesity rate exceeds $10 \%$ for both genders and has more than doubled during the past 40 years. ${ }^{202}$ Compared with those with normal BMI, morbidly obese patients have a significantly increased risk of postsurgical complications. ${ }^{203}$ Especially in the elective setting, controlled weight loss should be a goal for all obese patients. A target weight should be set and consultation to a nutritionist or a medical weight loss clinic should be strongly considered 


\section{Frailty}

Rather than age, the concept of frailty has evolved to describe the state of increased vulnerability resulting from a decline in physiological reserve and function across multiple organ systems, such that the ability to withstand stressors is impaired. Multiple studies have shown a strong association between frailty and adverse peri-operative outcomes. 115,204,205 Frailty can be assessed using a wide range of scales ${ }^{206}$ and tests, including the timed up and go 207 and the six minute walk test ${ }^{208}$. As the incidence of diverticulosis and diverticulitis increases with age, a large number of patients considered for sigmoid resection for diverticulitis will be found to be frail. Small studies are supportive of multimodal interventions. ${ }^{209}$ These include preoperative consultation with physical and occupational therapist, better known as "re-hab" as well as geriatric consultation in the perioperative period.

\section{Nutrition}

Preoperative malnutrition in the patient with diverticulitis can be a significant issue. The disease process can induce anorexia and fear of food, as it exacerbates symptoms. Patients being considered for sigmoid resection should be assessed for malnutrition. Based upon expert consensus, a diagnosis of malnutrition requires that the patient exhibit two or more of the following: Insufficient energy intake, weight loss, loss of muscle mass, loss of subcutaneous fat, localized or generalized fluid accumulation that may sometimes mask weight loss, and/or diminished functional status as measured by handgrip strength. ${ }^{210}$ In any patient with suspected malnutrition, protein status should be assessed with serum albumin, transferrin and prealbumin. Low serum albumin $(<2.2 \mathrm{~g} / \mathrm{dL})$ is a marker of a negative catabolic state and a predictor of poor outcome. ${ }^{211}$ For any patient with identified malnutrition, supplementation should be initiated. The first choice for supplementation is enteral. Perioperative immunonutrition seems to be the best approach to support malnourished patients with cancer, with an observed reduction in complications and length of stay. ${ }^{212}$ Consultation with a nutritionist is recommended. Patients with identified malnutrition who need to undergo urgent or emergent surgery, avoidance of an anastomosis (via Hartmann's procedure) or protection of the anastomosis (via diverting loop ileostomy) should be strongly considered. This group of patients should be screened early for postoperative total parenteral nutrition (TPN).

\section{Smoking}

Smoking has long been identified as a risk factor for post-operative wound infection, pneumonia and other post-operative complications. ${ }^{213,214}$ While previous studies have suggested increased complication in patients quitting less than 8 weeks before surgery, this has been disproven in more recent studies. ${ }^{215}$ Cessation of smoking at any time point outside 72 hours is beneficial for all smokers. But smoking cessation is no easy task. A combination of smoking cessation counseling, behavioral therapy and medical therapy are all important in helping patients quit. Urinary cotinine testing can be employed to confirm smoking cessation in select patients where self-reporting is questioned. \{Connor Gorber, $2009 \# 22\}$ 


\section{Medical}

Diabetes Mellitus-Diabetes mellitus is a common chronic disorder, affecting over $9 \%$ of the United States population. Poor perioperative glucose control may be the most important risk factor for development of SSI as well as other surgical complications, including death. 216,217 Because of this, glucose management in the pre-operative setting as well as the perioperative setting is paramount. In preparing patients for surgery, a baseline $\mathrm{HgBA} 1 \mathrm{C}$ should be obtained. Consultation with the patient's endocrinologist or PCP should be conducted to endure that glucose control is optimized before surgery. In the peri-operative period, tight glucose control with either an insulin drip or a sliding scale of insulin should be achieved.

Congestive Heart Failure-Heart failure (HF) is a common diagnosis requiring evaluation and treatment in patients undergoing abdominal surgery. The high burden of hypertension, diabetes, and coronary artery disease in the general population has increased the prevalence of HF. HF is a major risk factor for adverse cardiac events, including death as well as readmission in patients undergoing noncardiac surgery. ${ }^{218}$ Furthermore, elderly patients with HF undergoing noncardiac surgery have substantially higher risks of postoperative mortality and hospital readmission. Functional status, along with other signs and symptoms of HF, including exertional dyspnea, fluid retention, and fatigue, should be assessed in the preoperative evaluation. While routine preoperative testing is not generally indicated, preoperative tests such as an electrocardiogram or echocardiogram should selected based upon clinical indication and likelihood of testing changing the decision to proceed with surgery and perioperative management. Cardiology consultation is an important part of preoperative management. Intraoperative management includes fluid management and hemodynamic monitoring and communication with the anesthesia team is critical. In the post-operative period, physicians should pay close attention to volume infusion and be vigilant for pulmonary edema.

Hepatic Dysfunction-The incidence of liver cirrhosis is increasing, and thoughtful perioperative management of these patients is crucial. Colorectal surgery in a patient with cirrhosis is associated with $26 \%$ mortality. Postoperative complications such as stoma complications and anastomotic leaks are unique to this cohort of patients. Patient with liver dysfunction should be risk stratified, usually with the assistance of a hepatologist. The two most widely used scoring systems to help predict the morbidity and mortality of patients with cirrhosis undergoing various types of surgeries are Child-Turcotte-Pugh (CTP) and Model for End-Stage Liver Disease (MELD) score. ${ }^{219,220}$ A higher MELD score of 15 or more is an independent predictor of mortality. ${ }^{221}$ Colorectal surgeries in patients with CTP class A are safe; however, patients with CTP classes B and C had higher morbidity and mortality especially if emergency surgery is needed. ${ }^{222}$ In the perioperative setting, special attention needs to be paid to nutrition, coagulopathy $\&$ thrombocytopenia, fluid management, infection and ascites. ${ }^{223}$ Transjugular intrahepatic portosystemic shunt may improve sequelae of liver cirrhosis and should be considered prior to elective or semielective surgery.

Pulmonary dysfunction-Pulmonary complications are well known to contribute significantly to overall operative morbidity and mortality. In an analysis of the NSQIP 
database, postoperative pulmonary complications occurred in 6 percent of 165,196 patients who underwent major abdominal surgery. ${ }^{224}$ Underlying pulmonary disease, including COPD, obstructive sleep apnea, and pulmonary hypertension need to be identified in the perioperative assessment. If patients do not have a preexisting pulmonologist, consultation is recommended. In the peri-operative period, fluid restriction, lung protective ventilation and hemodynamic monitoring are extremely important. In the post-operative period, strategies such as incentive spirometry, early mobilization and multimodal pain control should be employed to reduce complications.

\section{Surgical Management}

In the past decade, Enhanced Recovery after Surgery (ERAS) pathways have standardized a number of best practices in the perioperative period. These include a minimal invasive surgical approach when appropriate, multimodal analgesia, restricted fluid administration, early feeding and early mobilization. Implementation of these bundles has been shown to decrease overall complications. 225

There is high-quality evidence that, in appropriate cases, when performed by properly trained personnel, laparoscopic treatment of colorectal conditions is beneficial compared with open surgery. In two separate multicenter RCTs laparoscopy was found to be superior to open resection in terms of short term outcomes (quicker return of bowel function, less blood loss, less postoperative pain, and shorter hospital lengths of stay). ${ }^{226,227}$ Several other RCTs have shown the benefit of a laparoscopic approach including reduced perioperative morbidity, including total morbidity, wound morbidity, nonsurgical morbidity, decreased time to pulmonary recovery, reduced use of narcotics, and improved short-term quality of life. ${ }^{228-230}$ In appropriately selected patients operated on by trained surgeons, a laparoscopic approach should be the operation of choice.

There are other important elements of an ERAS pathway. A standardized pathway for multimodal analgesia should be agreed upon by both the anesthesia and surgery teams. A number of well-done studies have demonstrated that minimizing opioids is associated with earlier return of bowel function and shorter length of stay. ${ }^{231-233}$ A number of techniques are available to limit opioid intake. Avoiding patient controlled narcotic analgesia pumps, utilizing epidurals and regional blocks, and scheduling narcotic alternatives, such as oral acetaminophen, nonsteroidal anti-inflammatory drugs (NSAIDs), and gabapentin, rather than giving them on an as-needed basis are all useful ways to decrease narcotic dependence. Early feeding, as defined by less that 24 hours after surgery should be offered to all patients. Early feeding has been shown to accelerate GI recovery and decrease length of stay. ${ }^{234}$ In addition, early mobilization should be employed in appropriate patients. Early and progressive patient mobilization is associated with shorter length of stay. ${ }^{235}$

\section{Summary}

In treating the patient with diverticulitis, it is imperative to identify a wide range of modifiable patient co-morbidities. Every attempt should be made to improve a patient's chance of successful surgery. This includes optimization of patient risk factors as well as 
tailoring the surgical approach and perioperative management. A positive outcome depends greatly on thoughtful attention to what makes a complicated patient "complicated".

\section{It's time to operate - what do you do?}

Despite a recent shift in the trend in management strategies, surgery for diverticular disease is increasingly common. \{Bharucha, 2015 \#31;Etzioni, 2009 \#37\} Operative management remains complex and depends on multiple factors including patient age, comorbidities, nutritional state, severity of disease, and surgeon preference and experience. Importantly, the status of surgery, elective versus urgent or emergent operation, is pivotal in decision-making, and treatment algorithms are divergent based on the acuteness of surgery.

Despite multiple operative approaches, there are important core principles of a surgical resection for diverticulitis. It is imperative that the entire sigmoid colon be resected, with the distal margin extending to at least the proximal rectum and possibly more distal in the case of secondary rectal inflammation, to ensure a negligible risk of recurrence. \{Thaler, 2003 \#601\} The proximal margin on the descending colon should also be without inflammation or thickening. While it is unnecessary to chase diverticula proximal to the sigmoid, the tissue incorporated into the anastomosis should be without diverticula due to an increased risk of anastomotic leak. Lastly, although high ligation of the inferior mesenteric artery is a central tenant of resection for malignancy, this technique diminishes the blood supply to the rectum and may increase the risk of anastomotic complications in a sigmoid resection for diverticulitis. \{Tocchi, $2001 \# 625$ \} This is especially true in older patients in whom compensation of blood flow from the middle and inferior hemorrhoidal arteries may be compromised.

\section{Acute surgical management}

Investigation into the appropriate surgical treatment of acute diverticulitis has grown and evolved as the incidence of diverticulitis has increased over the past century. Historically, surgery consisted of a three-stage approach: diverting loop colostomy followed by resection of the diseased segment and lastly colostomy reversal. In the 1970's, the advantages of a primary sigmoid resection at the first operation were recognized, and a two-stage approach became the most common surgical approach, leaving a colostomy and a long Hartmann's. As there was increasing attention given to high rates of a permanent colostomy and morbidity with a two-stage procedure, there has been increased interest in a sigmoid resection with primary anastomosis with or without a diverting loop ileostomy at the first operation.

\section{Loop colostomy}

Operative treatment for acute diverticulitis was described as early as 1910. Initially, an abdominal washout and drainage with oversewing of the colonic perforation was advocated. \{Cirocchi, 2017 \#626\} However, due to challenges in performing colonic repair secondary to difficult visualization of the perforation and poor quality of surrounding inflamed tissue, fecal diversion with a proximal colostomy as an initial operation became the gold standard. \{Smiley, 1966 \#628;Staunton, $1962 \# 627\}$ 
Although a loop colostomy has the potential advantage of short operative time and avoidance of significant bowel edema and adhesions in the pelvis, it does not remove the septic source. Multiple randomized controlled trials (RCTs) have demonstrated that primary resection of the sigmoid has superior outcomes compared to diversion alone, and has therefore been replaced by the Hartmann's procedure as the most common operative treatment. \{Kronborg, 1993 \#630;Zeitoun, 2000 \#629\}However, there are some circumstances when diversion without resection can still be considered. These include unstable patients, surgeon comfort in the operating room, a hostile operative field in which safe resection cannot be performed, or concern for locally advanced colon cancer.

Technical considerations-Most commonly a transverse colostomy is created, although some advocate for use of the sigmoid colon due to concern for residual fecal residue in the descending colon. The operation is generally performed via a midline incision. The peritoneum is irrigated, and if gross perforation from the colon is identified, attempt at primary repair can be performed. The colon is then mobilized, and only if necessary, the hepatic or splenic flexure may also be taken down to allow the selected area of colon to reach the abdominal wall; preservation of the lateral attachments and splenic flexure will facilitate the next stage of sigmoid resection and primary anastomosis. A window is made at the mesenteric border of the colon. An appropriate area to make the stoma is chosen, if the patient was not marked preoperatively, and after incision through the skin and fascia, the loop of bowel is delivered through the abdominal wall. There should be no tension during this part of the procedure to avoid mesenteric tears and later retraction of the ostomy. Some advocate for use of a stoma support rod, although this step has not been shown to reduce postoperative complications. \{Franklyn, 2017 \#632; Whiteley, 2016 \#631\} A drain is frequently placed in the pelvis. The midline incision is then closed followed by maturation of the colostomy.

\section{Sigmoidectomy with colostomy (Hartmann's procedure)}

The current standard of care in the surgical treatment of acute diverticulitis is sigmoidectomy. \{Feingold, 2014 \#219\} Although first described by Mikulicz in the early 1900s for treatment of cancer, sigmoid resection with terminal colostomy and rectal stump left in situ is most commonly referred to as a Hartmann's procedure (HP). \{Cirocchi, 2017 \#626\} This operation gained in popularity in the 1980s after a systematic review in 1984 showed decreased mortality with primary sigmoid resection compared to colostomy without resection. \{Krukowski, $1984 \# 633$ \} The additional potential benefit of resecting an incidental cancer was also noted. This review was further supported by two RCTs. \{Kronborg, 1993 \#630;Zeitoun, 2000 \#629\} The HP is now the benchmark to which alternative procedures for diverticulitis are compared.

After the initial resection for septic source control, colostomy reversal to restore intestinal continuity can be performed. This usually occurs $2-6$ months after the first operation. The risk of morbidity and mortality after the second procedure is not trivial and frequently requires re-do laparotomy. \{Aydin, $2005 \# 88$ \} For these reasons, careful consideration of the patient's initial severity of disease and overall condition must be taken. 
Technical considerations-Conventionally, the operation is approached via a midline incision. The abdomen is first explored to confirm the diagnosis and assess the severity of disease. The diseased bowel is then mobilized. Mobilization is best facilitated by starting the dissection at proximal, non-diseased bowel to best identify the appropriate tissue planes. Care must always be taken to identify and avoid injury to the ureter. The proximal and distal resection margins are identified, and the bowel divided. The rectum is most commonly divided with a stapler, and the stump may be oversewn if there is concern about the staple line. The mesentery is divided, which may be difficult due to thickening and inflammation. Division with small bites close to the bowel may be helpful. The proximal end of colon is then brought up through the abdominal wall after incision through the skin and fascia. Frequently a drain is placed in the pelvis. The midline incision is closed and colostomy matured.

\section{Sigmoidectomy with primary colorectal anastomosis}

Up to $45 \%$ of patients will never undergo attempt at colostomy reversal after HP \{Vermeulen, $2009 \# 634$ \}, and those who do are at significant risk of major morbidity including surgical site infection and anastomotic leak. \{Aydin, 2005 \#88\} Due to these risks, the possibility of primary resection and colorectal anastomosis with or without a diverting ileostomy has more recently been proposed. Most studies addressing this question have been retrospective and limited by selection bias, but have largely supported primary anastomosis in select patients. \{Constantinides, 2007 \#635;Salem, 2004 \#23\} A small multicenter RCT comparing primary anastomosis (PA) with diverting ileostomy to HP for patients with Hinchey III or IV diverticulitis has been performed. \{Oberkofler, 2012 \#636\} Patients were randomized to 2 groups; 30 underwent $\mathrm{HP}$ and $32 \mathrm{PA}$. There were no differences in mortality ( $13 \%$ vs $9 \%$, respectively) or overall complications (67\% vs $75 \%$, respectively), but diverting ileostomies were more likely to be reversed than end colostomies $(90 \%$ vs $57 \%$ ). There were also no major complications after ileostomy reversal, compared to $20 \%$ after colostomy reversal, and hospital costs and length of stay were also significantly reduced following ileostomy reversal versus colostomy reversal. A more recent larger RCT, the DIVERTI trial, enrolled 102 participants and had similar results. \{Bridoux, $2017 \# 637$ \} There were no differences in mortality (7.7\% HP vs $4 \% \mathrm{PA}$ ) or major morbidity (39\% HP vs $44 \%$ PA), but more PA patients (96\%) underwent stoma reversal compared to HP (65\%).

These data suggest that PA is preferable to HP in select patients with acute diverticulitis. Patients with hemodynamic instability, immunocompromised state, feculent peritonitis, severely edematous or ischemic bowel, or significant malnutrition are poor candidates. Although most studies evaluated PA in combination with diverting ileostomy, one third of patients in the DIVERTI trial were not diverted. Given the paucity of evidence, the decision to divert after colorectal anastomosis is at the discretion of the operating surgeon. Patient factors including severity of disease, tissue quality, and comorbidities should be considered.

Technical considerations-In addition to the general principles of resection listed above, there are several additional considerations when creating a colorectal anastomosis. The anastomosis must be tension-free, which usually requires mobilization of the splenic flexure and sometimes rectal mobilization. A stapled anastomosis is most frequently 
performed but may also be hand-sewn. In both circumstances, intraoperative air testing by occluding the proximal bowel and inflating the anastomosis while under saline should be completed to reduce the risk of anastomotic leak. \{Beard, $1990 \# 638$ \} If air bubbles are seen, the anastomosis should be revised or diverting stoma created.

\section{Other considerations}

Laparoscopic colon resection-The role of laparoscopy in the acute setting remains controversial. A small retrospective review studied 42 patients who underwent laparoscopic or open surgery. \{Letarte, 2013 \#639\} Both Hartmann's procedures and resection with PA were included. There were no significant differences in baseline characteristics between the laparoscopic and open groups. Open operations were on average 36 minutes shorter but associated with significantly increased overall morbidity and length of hospital stay. A recent Cochrane review, however, concluded that urgent laparoscopic surgical resections take longer and show no benefit with regards to major and minor complications, postoperative morbidity or mortality, reoperations, and time to diet. \{Abraha, 2017 \#641\} Additional evidence to determine the safety and effectiveness of laparoscopic surgery for acute diverticulitis is needed.

Ureteral stents-Routine use of ureteral stents is not recommended. \{Feingold, 2014 \#219\} Ureteral stenting aids in the detection of ureteral injury but does not prevent injury. \{Pokala, 2007 \#647\} Placement increases costs and operative times, and there is risk of injury during instrumentation. Selective use may be beneficial in certain patients, such as those who are morbidly obese, who have had prior abdominal operations or radiation, and in those in whom preoperative imaging suggests abnormal anatomy.

\section{Elective surgical management}

\section{Technical considerations}

In contrast to acute operations, approximately $95 \%$ of elective resections are performed with PA. \{Masoomi, $2011 \# 648\}$ Additionally, the laparoscopic approach is preferred when feasible. \{Feingold, 2014 \#219\} Multiple RCTS have shown laparoscopy in the elective setting decreases blood loss, post-operative pain, complications, and hospital length of stay after colon resection. \{Gervaz, 2011 \#642;Klarenbeek, 2011 \#599\} Surgical decisionmaking still depends on patient factors, notably the indication for surgery. The presence of active inflammation, colovesical, colovaginal, colocutaneous, or coloenteric fistula, and colonic dilation secondary to diverticular stricture should be considered when deciding to take a minimally invasive approach or perform an anastomosis.

Minimally invasive surgery-In conventional or "straight" laparoscopy, all portions of the case, including the anastomosis, are performed with small 5-12mm trocars. One of these trocar sites is expanded to allow extraction of the specimen. A hand-assisted technique is also commonly utilized. A hand port is placed through either a lower midline or Pfannenstiel incision to assist in dissection, and the specimen is subsequently extracted via this incision. These approaches yield similar outcomes, although hand assistance may decrease operative time and conversion rate. $\{$ Chang, $2005 \# 650$;Marcello, $2008 \# 649$ \} More recently, robotic 
surgery has been increasingly utilized due to its theoretical advantages including threedimensional high definition optics, stable platform, and availability of articulating instruments in 7 degrees of freedom. These enhancements may be especially beneficial when handling hard and inflamed tissue. Outcomes are comparable to laparoscopic surgery and may also decrease the risk of conversion. \{Maciel, 2014 \#651\} These potential advantages must be weighed against the increased costs and operative times associated with robotic assistance. Until further data is available, the choice of minimally invasive approach should be based on surgeon preference.

Dissection can be carried out in either a lateral-to-medial or medial-to-lateral approach. In the latter, a plane is made below the IMA, and the retroperitoneum is swept downward. After identification of the ureter, the bloody supply is divided. The attachments between the mesocolon and the retroperitoneum and Gerota's fascia are further separated. The lateral attachments are divided, extending to the splenic flexure as necessary. Once mobilized, the appropriate margins are chosen and resection performed.

\section{Complicated diverticulitis}

Fistulizing disease-The presence of a fistula, most commonly a colovesical fistula, can significantly increase the difficulty of an operation. Frequently the sigmoid colon is tethered into the pelvis. In these cases, the fistulas can be divided with either sharp or blunt dissection. It is often not necessary to close a bladder or vaginal wall defect or to excise the surrounding fibrotic area given these areas commonly heal once the inflamed colon is removed. However, a methylene blue test by instilling $600 \mathrm{cc}$ of normal saline with methylene blue into the bladder can be useful to see if the fistula tract has a large enough diameter that requires closure. In this case, a primary two-layer closure with absorbable suture should be performed, and a Foley catheter left in place for at least two weeks for maximal drainage. An omental flap can also be used to cover the region.

Stricture-Patients with strictures secondary to recurrent inflammation may present with either acute or chronic large bowel obstruction. A primary anastomosis can frequently be performed, but a Hartmann's procedure should be considered when the colon is significantly dilated or has signs of ischemia.

\section{Other considerations}

Bowel preparation-Oral mechanical bowel preparation alone does not affect the rate of wound infection or anastomotic leak after open colon surgery. \{Guenaga, 2011 \#652\} Bowel preparation for laparoscopic surgery lacks sufficient data, and the utility of its use is inferred from literature on open surgery. Mechanical bowel preparation is still commonly prescribed pre-operatively; the decision to implement is per surgeon preference.

The literature does support use of non-absorbable oral antibiotics, such as erythromycin, neomycin, and metronidazole. A large Veteran Affairs study assessed rates of surgical site infection in nearly 10,000 patients. \{Cannon, $2012 \# 653$ \} Those who received no bowel preparation $(18.1 \%)$, or mechanical bowel preparation only (20\%) had significantly more post-operative infections than those who received either oral antibiotics alone $(8.3 \%)$ or in 
combination with mechanical bowel preparation (9.2\%). Other studies have shown that oral antibiotic preparation may also decrease organ space infections, ileus, and hospital length of stay. \{Englesbe, 2010 \#655;Toneva, 2013 \#654\} Non-absorbable oral antibiotics do not increase risk of clostridium difficile infection. \{Krapohl, 2011 \#657\} The most current guidelines recommend combined mechanical and oral antibiotic bowel preparation for elective colon and rectal resections. \{Migaly, $2019 \# 658$ \}

Ureteral stents-Ureteral injury occurs in less than $1 \%$ of elective colon surgeries. \{Masoomi, 2011 \#648\} Routine use of ureteral stents is not currently recommended due to increased operative times, cost, and lack of evidence demonstrating usage prevents injury. \{Feingold, 2014 \#219;Pokala, 2007 \#647\} Placement is at the discretion of the surgeon but may be beneficial in patients who are morbidly obese, have had prior abdominal operations or radiation, or have abnormal anatomy on preoperative imaging.

Summary-Surgical management of diverticular disease continues to evolve as incidence increases. Resection of diseased bowel to healthy proximal colon and rectal margins remains a fundamental principle of treatment although the operative approach may vary. Multiple patient and disease factors must be considered, and the advent of new technology has made operative decision-making even more complex. Careful preoperative planning and perioperative choices are critical for successful post-operative outcomes.

\section{Colostomy Closure after Hartmann's for Diverticulitis: Minimizing the Misery}

\section{Background}

Sadly, between $20-50 \%$ of patients treated with sigmoid resection and an end colostomy after an initial severe bout of diverticulitis will never be reversed to their normal anatomy. \{David, 2009 \#81;Horesh, 2017 \#78;Mealy, 1996 \#80;Mosdell, 1991 \#77;Resio, 2018 \#79\} The reasons for high rates of permanent colostomies are multifactorial. \{Horesh, 2017 \#78; Roque-Castellano, 2007 \#2 \} While many variables are related to the clinical status of each patient, the morbidity and surgical challenges of a colostomy reversal cannot be trivialized. \{Horesh, 2017 \#78\} There are, however, a number of tips that are worth reviewing to help obviate the challenges of colostomy reversal, from the initial presentation to the colorectal anastomosis. Our aim is to provide a roadmap for safe and efficient colostomy reversal.

\section{The Initial Presentation of Acute Diverticulitis}

During the acute inflammatory phase of diverticulitis, avoidance of surgery should be the primary goal. This is intuitive, yet segmental resection with an end colostomy is performed all too frequently; $64 \%$ of surgeries are performed in the acute presentation of in one population-based study. \{Li, $2014 \# 83\}$ Conversely, non-operative management may be the less conservative option. In all cases of complicated diverticulitis, fluid resuscitation, bowel rest, and IV antibiotics are the staple of initial medical management. If an associated fluid collection is present, consultation with an interventional radiologist should be initiated early in an admission. There is low-level, but growing, evidence suggesting that antibiotics may 
not be necessary in all cases, even with Hinchey $1 \mathrm{~b}$ diverticulitis, although this is currently not standard of care. \{Daniels, 2017 \#341; Tandon, 2018 \#85\} Parenteral nutrition should be considered early, recognizing that the process likely started days before the hospital presentation.

Unless clear signs of peritonitis are present, a trial of non-operative management should be considered with the aim of performing a single-stage operation at a later date if indicated. Patients with localized signs of peritonitis can often be managed with IV antibiotics and resuscitation plus bowel rest. Conversely, hemodynamic unstable patients and nonresponders to initial resuscitation will require emergent operative intervention.

If the initial CT scan does not show a drainable fluid collection and the patient is in a holding pattern, with minimal signs of improvement, after 5-7 days, reimaging to assess for evolution of their disease with a possible drainable fluid collection should be considered.

\section{Surgery at the Initial Presentation of Acute Diverticulitis}

There is mounting evidence that primary colorectal anastomosis with or without a diverting loop ileostomy is safe with Hinchey III and IV diverticulitis at the initial operation. \{Acuna, 2018 \#87;Lambrichts, 2019 \#86\} Studies report conflicting results. \{Aydin, 2005 \#88;Aydin, $2006 \# 89$;Resio, $2018 \# 90\}$ Each case is unique, and there are no clear guidelines on this practice. The operating surgeon must balance the benefit of avoiding another major operation with the risk of an anastomotic leak. Thus the decision to perform a Hartmann's procedure is at the surgeon's discretion.

When exploration and resection are necessary, there are several key steps to consider that reduce the difficulty of future colostomy reversal. When possible, leave a long rectal stump even if it means a leaving a segment of distal sigmoid; rarely is it necessary to go below the pelvic brim. If the rectum is divided too distal, it will retract, atrophy, and reperitonealize, rendering future recognition of the stump and anastomosis challenging. Mark the rectal stump with one or two permanent monofilament sutures on either side of the staple line. Leave these sutures long to assist with identification of the rectal stump during the reversal. Lastly, if possible, consider tacking the rectum to the peritoneum to prevent retraction into the pelvis.

There is good data to support the use of Seprafilm [Genzyme Corp., Cambridge, MA] in the prevention of post-operative abdominal adhesions. \{Becker, 1996 \#91;Kumar, 2009 \#92\} However, the data may not be generalizable to acute diverticulitis with contamination of the peritoneal cavity.

Avoid mobilization of the splenic flexure at the initial operation. Adhesions that develop increase the complexity of future splenic flexure mobilization. Similarly, avoid removing more colon than necessary, as doing so increases the difficulty of performing a tension-free anastomosis.

One challenge of a colostomy reversal is the unknown regarding the first operation. Frequently, the operating surgeon is not the same surgeon who performed the initial surgery. To avoid surprises, the surgeon should remain diligent in their investigation of the patient's 
anatomy, pathophysiology, comorbidities, and prior medical care. Review of prior operative notes, pathology reports, CT scans, and colonoscopy reports is paramount. The initial pathology report should confirm the expected disease process.

A flexible sigmoidoscopy is recommended to evaluate for additional pathology and to verify the rectal stump length and condition. Alternatively, a gastrografin enema provides similar information. Either study is useful to evacuate retained feces or scybala, thereby easing the passage of the EEA stapler at the future operation. The proximal colon should be cleared endoscopically prior to colostomy closure except when a patient is up-to-date with their screening.

A preoperative CT scan can help plan the best operation. Of particular interest is the location and configuration of the rectal stump, Figure 6, and the presence of hernias - parastomal or midline - which may influence the approach to closure and whether a herniologist's assistance will be necessary.

\section{Timing of Colostomy Takedown}

The debate on the best timing for a colostomy takedown continues. The lack of prospective studies and the inherent bias of retrospective reviews continue to fuel the discussion.

An early takedown of a colostomy has important disadvantages. Acute inflammatory adhesions contribute to a difficult dissection with increased risks of enterotomies and stump identification. As the inflammatory process moves to the remodeling phase, adhesions soften, generally leading to a straightforward and safe adhesiolysis. Conversely, waiting too long may increase the fibrotic adhesions encountered, making rectal stump identification and mobilization more challenging. \{Roberts, 2014 \#93\}

Six months is generally chosen as the safest time to proceed when adhesions may be at their softest allowing for a more favorable dissection. There are retrospective studies supporting both early and delayed reversal, all of which are subject to selection and timing bias.

Early Reversal-Using a recent multistate claims database, Resio et al. found improved outcomes with earlier reversal in selected patients, however, there were also disparities in timing by insurance-status and race confounding the study's results. \{Resio, 2018 \#79\} Another population-based study in Washington state reported early reversal was associated with a decrease risk of a second stoma. \{Salem, 2005 \#94\} Both studies have limited granular information that is inherent when using insurance claims data.

Delayed Reversal-A single center review of 48 patients who underwent a Hartmann's takedown found no difference between early and late operations regarding anastomotic leaks or morbidity, however, they noted a significantly higher number of accidental enterotomies in the early reversal group. \{Keck, $1994 \# 95$ \} Another single center review found a striking difference in anastomotic leaks in reversals before vs. after six-months from the initial operation, $13 / 40$ vs. 0/40, respectively. \{Pearce, $1992 \# 96$ \} The list of small studies advocating one direction or the other is seemingly endless. \{Albarran, 2009 \#97; Horesh, 2017 \#78;Khan, 1994 \#98;Mosdell, 1991 \#77\} 
Wait Six Months!-One of the advantages of waiting at least six months prior to reversal is the opportunity to optimize comorbidities and nutritional status prior to colostomy takedown. In general, these patients are septic at initial presentation necessitating an emergent exploration. Upon discharge, they often require admission to an acute rehabilitation facility and may be functionally impaired. Similarly, they may require optimization of their cardiopulmonary and endocrine status prior to any planned intervention. Additionally, modifiable factors may need correction, i.e. tobacco abuse has strong associations with complications after colostomy reversal. \{Fleming, 2009 \#99\} Prehabilition should be considered, especially for the frail patient. \{Gillis, $2018 \# 100\}$

\section{Surgical Approach}

Studies evaluating the surgical approach for a colostomy takedown procedure are retrospective and inherently flawed by selection bias. \{Arkenbosch, 2015 \#102;Cellini, 2013 \#104;De'angelis, 2013 \#106;Guerra, 2019 \#101;Holland, 2002 \#105;Kwak, 2018 \#103\} Two studies using NSQIP, not limited to diverticulitis patients, showed a decrease in morbidity with the laparoscopic approach compared to open surgery. \{Arkenbosch, 2015 \#102;Cellini, 2013 \#104\} In a small series of Korean patients, laparoscopic reversal was associated with an earlier return of bowel function and less blood loss in the OR. \{Kwak, $2018 \# 103\}$

Laparoscopic-assisted transanal Hartmann's colostomy reversal has been reported by a single center with modest success. \{Bravo, 2016 \#107;Trepanier, 2017 \#108\} In the first ten patients reported, a diverting loop ileostomy was created and iatrogenic enterotomies were made in 5 and 4 patients, respectively. The authors indicate this is a new approach that may help with challenging cases, i.e. hostile pelvis or difficult rectal stump identification. \{Trepanier, $2017 \# 108\}$ Likewise, the technique of robotic-assisted surgery for a colostomy takedown has been described with a stapled \{De'angelis, $2013 \# 106\}$ and hand-sewn anastomosis. \{Kudsi, $2019 \# 110\}$ Results using robotic-assistance are limited to case reports.

Minimally invasive techniques can be considered if expecting minimal adhesions, i.e. successful laparoscopic approach \{Celentano, $2018 \# 111$ \} or minimal contamination at initial surgery, and when a long rectal stump is intra-peritoneal. Frequently, however, the initial presentation, which required an emergent surgery, is better approached open due to the nature of expected adhesions and the status of the rectal stump. Ultimately, the surgical approach will be a personal decision by the operating surgeon based on his or her experience.

\section{The Operation}

Colostomy takedown operations are challenging surgeries. The surgeon should anticipate and appropriately plan for a long and difficult operation. The patient should undergo a full antibiotic bowel preparation. It may be necessary to mark pre-operatively for a potential ileostomy in the event of a hostile pelvis, or when there is a contracted and coiled rectal stump. 
Placement of ureteral stents should be considered, especially if the surgeon anticipates a difficult dissection due to a history of severe pelvic sepsis, hydronephrosis, or unclear anatomy on CT scan. Stents do not prevent ureteral injury but do facilitate recognition of the ureters and early identification of injury facilitating repair. Regardless of the presence of stents, both ureters should be identified in all cases as early as possible. \{Roberts, 2014 \#93\}

After ensuring the patient is appropriately padded and in lithotomy position, a rectal washout may be necessary if pre-operative endoscopic or barium enema evaluation was not performed.

The operation can be broken down into five steps: 1) adhesiolysis, 2) mobilization of the rectal stump, 3) mobilization of stoma and proximal colon, 4) the anastomosis, and 5) abdominal wall closure.

\section{Step 1) Adhesiolysis}

Not too much to advise for the trained surgeon. Starting the operation fresh, use good lighting, including a headlight, and having senior-level help is essential.

\section{Step 2) Define and mobilize the rectal stump.}

Rectal stump identification can, at times, be the most challenging portion of the case. Prior adhesions, a contracted and coiled stump, abscess or a fixed, indurated pelvis can all contribute to making this a challenge. Marking sutures at the first operation facilitate stump identification, as the prior staple line is not reliable. Other options for stump location include: 1) uterine manipulators; 2) ureteral catheters; 3) identification pre-sacral plane and mobilization of the rectum; and 4) define the rectum with EEA sizers or a proctoscope.

After the rectal stump identification, the length, tortuosity, and degree of rectal stump atrophy need to be addressed. Frequently, the top of the rectal stump near the old staple line is fibrotic, and non-pliable, i.e. not adequate for an anastomosis. The posterior midline is generally the safest approach to initial rectal dissection. When the stump is contracted, mobilization below the peritoneal reflection may be required. The rectum should be mobilized until it is noticeably soft and pliable. Finally, the old staple line should be excised down to healthy rectum.

\section{Step 3) Evaluate the status of the proximal colon and ostomy takedown.}

Once the rectum is appropriately prepared, and suitable for an anastomosis, attention is turned to the colostomy and proximal colon. The stoma is released from its mucocutaneous junction and brought into the peritoneal cavity. Frequently, the distal 3-4 cm of the colon is not suitable for anastomosis and needs to be resected. It is not necessary to resect all proximal diverticula only to ensure no diverticula are present within and distal to the anastomosis.

\section{Step 4) The Anastomosis.}

Part 1: Will it reach?-There are critical steps to consider when assessing for adequate length for a tension-free anastomosis. These include: 
1. Splenic flexure mobilization and omental dissection off the transverse colon;

2. Inferior mesenteric artery (IMA) ligation. Proximal division of the superior hemorrhoidial artery, first, to avoid ischemia to the left colon vis-à-vis the left colonic artery, and only if necessary, the IMA.

3. Inferior mesenteric vein (IMV) ligation at the inferior border of the pancreas;

4. Mobilization of the rectum (previously discussed); and

5. Preservation of marginal artery of Drummond.

Part II: What if the colon will not reach?-When excess colon was resected, either inadvertently or due to the disease process, two additional options remain for creating an anastomosis - retroileal pull-through and the Deloyers Procedure. The retroileal pullthrough is a maneuver that involves creation of a ileal mesenteric window to facilitate a transverse colorectal tension-free anastomosis, Figure 7. \{Rombeau, $1978 \# 112\}$ The Deloyers Procedure can be utilized when a retroileal pull through is not feasible. The technique involves complete mobilization of the right colon and small bowel mesentery to the duodenum, division of all the middle colonic vein branches, rotation of the colon counterclockwise, and \pm appendectomy, Figure 8 . \{Deloyers, $1964 \# 113$ \} For both of these maneuvers it is essential to preserve the marginal artery in order to prevent ischemia to the colon conduit.

Part III: The Anastomosis.-There are a number of different techniques available for performing a colorectal anastomosis. A single stapled, double purse-string end-to-end anastomosis is our preferred technique as there are no crossing staple lines, Figure 9. A purse-string on the rectal stump may be impractical when a short stump is deep in the pelvis. In select cases, stapled rectal stump with a Contour [Ethicon Inc., Somerville, NJ] or TA stapler [Covidien, Dublin, Ireland] is the better choice, Figure 10. If there is a size mismatch between the rectum and colon, a side-to-end anastomosis, i.e. Baker technique, is a possible option. The anvil can be placed on the colonic or rectal side of the anastomosis. The upsidedown technique can be utilized when there is significant atrophy of the rectum, which may cause perforation with passage the EEA stapler [Covidien, Dublin, Ireland], Figure 11.

Assessing the rectal stump lumen caliber and length with sizers can help determine if the rectum needs further mobilization to allow passage of the EEA stapler without difficulty. Progressively larger sizers are used to gently dilate the rectum but care is taken to avoid "cracking" the rectal wall. If endoluminal resistance is a concern, consider downsizing to the $25 \mathrm{~mm}$ EEA stapler. One can consider passing the stapler with the anvil attached to ease the passage to the top of the rectal stump when the stump is open, e.g. during the double pursestring technique.

Passing the stapler through the anterior rectal wall for anastomosis should be avoided due to the risk of incorporating anterior structures including the bladder, seminal vesicles, prostate, or vaginal wall. When the surrounding anatomy is unclear, consider filling the bladder and using vaginal manipulators. Furthermore, tension and ischemia should be avoided to prevent leak and stricture. 
After the EEA stapler is gently removed, the tissue rings, i.e. "donuts," are inspected for thickness and integrity. There is no exception for not testing the anastomosis for leaks. The lumen should be visualized for bleeding and integrity at the anastomosis staple line. If there is a positive leak test, proximal diversion or anastomotic reconstruction should be considered. Positive leak test managed by suture repair-only was associated with a $9 \%$ leak rate compared to $0 \%$ for the diversion or reconstruction group in a recent series of patients. \{Mitchem, $2018 \# 114$ \} The size and location of the leak and ease of access to the anastomosis are factors which will influence the decision for primary repair versus reconstruction and a covering stoma.

Closed suction drainage should be considered for a low anastomosis; however, there is no data to support this practice.

\section{Step 5: Closing the abdomen.}

It is not uncommon for this patient population to have a midline and/or a parastomal hernia. Providing a durable hernia repair during the same operation as the colostomy takedown can be challenging. The adage, mesh should not be placed in an infected field, still rings true. Newer slowly absorbable biosynthetic meshes, i.e. BIO-A [Gore and Associates, Inc., Flagstaff, A] or Phasix [Davol, A Bard Company, Warwick, RI] are options, and they provide an intermediate option between biologic and permanent mesh. For complex closure of the abdominal wall, involving a hernia specialist in the preoperative planning is encouraged.

\section{Summary}

Colostomy takedown is an under-appreciated and challenging operation. Restoration of bowel continuity is not performed in up to $50 \%$ of patients after their initial surgery for diverticulitis. It is essential to wait six months after the initial operation to minimize the risk of enterotomies and other complications. Preoperative planning is critical; review the initial operative note and defining the anatomy prior to reversal. When a complex abdominal wall closure is necessary, consider consultation with a hernia specialist. Open surgery is the preferred surgical approach for the majority of colostomy takedown operations. Finally, consider ureteral catheters, diverting loop ileostomy, and be prepared for all anastomotic options in advance.

\section{Is there a role for laparoscopic lavage in diverticulitis?}

\section{Introduction}

Since its inception in the late 90's, laparoscopic lavage has been recognized as a novel treatment modality in the management of complicated diverticulitis; specifically, Hinchey III (purulent) diverticulitis. Over the last decade, it has been the subject of several randomized controlled trials, retrospective studies, systematic reviews as well as cost-efficiency analyses. Despite being the subject of much debate and controversy, there is a clear role for laparoscopic lavage in the management of acute diverticulitis with the caveat that patient selection is key. 


\section{Surgical Technique}

It is interesting to note the variation and evolution of the lavage technique over the last 10 years. Gregori at al recently conducted a comprehensive review of the available data regarding surgical technique for laparoscopic lavage. \{Gregori, 2019 \#665\} Twenty-eight studies were included in the analysis. All studies commenced antibiotics pre-operatively according to local protocols and were continued for 5-7 days post operatively. In terms of operative technique, an open Hasson entry was used in most institutions. Trocar placement and number of trocars vary as would be expected according to surgeon preference. The major technical question is whether the sigmoid colon should be mobilized and any adhesions or omentum dissected in order to identify a perforation. This option allows the surgeon to wash any interloop abscesses or pockets of fluid that may otherwise remain inaccessible. In addition, identification of a perforation allows for a definitive procedure such as conversion to a colonic resection or over-sewing the perforation and placing an omental patch. \{Sorrentino, 2015 \#499;Swank, 2013 \#498; White, $2010 \# 500\}$ On the other hand, some recommend leaving the sigmoid colon and any adhesions alone in order to encourage a perforation to seal. In the context of purulent peritonitis, it stands to reason that there is no overt perforation as there is absence of fecal contamination. This concept is key to the success of laparoscopic lavage as an unsealed colonic perforation requires some form of intervention (colporrhaphy or resection). Uncertainty regarding the presence or absence of feculent peritonitis and the management of an obvious colonic perforation at laparoscopy may be responsible for the higher rates of complications seen in some studies. \{Vennix, $2015 \# 486$ \} It is worthwhile to note the variation in surgical technique between the most recent studies. For example, the LADIES trial protocol advises a laparoscopic assessment of the whole abdomen with an attempt to identify the site of the diverticular perforation by careful dissection of omentum or small bowel away from the sigmoid colon. \{Swank, 2010 \#485\} However, they so comment that any adhesions should be left in place if clearly adherent. In the SCANDIV trial, \{Schultz, $2015 \# 497\}$ two drains are placed whereas the DILALA trial advises a saline washout with placement of a drain but also avoided identification of a perforation. \{Angenete, $2016 \# 496$ \}

The LLO study (multicenter but retrospective) is one of the largest studies to scrutinize surgical technique. \{Binda, $2018 \# 495$ \} From their analysis, extensive adhesiolysis was a risk factor for failure of laparoscopic lavage. Again, they suggest that adhesiolysis may transform a covered perforation into a free perforation. Indeed, in their cohort, if a free perforation was identified at laparoscopy, patients were converted to an open operation in a very small number the perforation was sutured. In this study, nearly $75 \%$ had a successful early outcome.

In one of the original reports of laparoscopic lavage, the abdomen was irrigated only and adhesions or a search for a perforation was not performed. \{O'Sullivan, 1996\#666\} This is based on the premise that this subset of patients presents with acute abdominal sepsis without feculent peritonitis. Clearly, any variation in surgical technique creates difficulties when attempting to compare the outcomes from various studies and trials.

In terms of the actual lavage procedure, the volume of fluid used varies in all studies ranging from 3L to over 20L. Most studies report irrigating until the effluent runs clear. In addition, 
the use of abdominal drains differed between studies. Drains were placed close to the sigmoid to identify a fecal leak or in the pelvis to avoid a pelvic collection. Duration of drainage and number of drains were according to surgeon preference. \{Schultz, 2017 \#484\} In terms of post-operative management, most patients were kept fasted and enteral nutrition introduced on the $4^{\text {th }}$ or $5^{\text {th }}$ post-operative day. Average length of stay in this review was approximately 8 days. \{Gregori, $2019 \# 665$ \}

The overall evolution of the lavage technique consists of an abdominal washout with warmed saline, avoidance of searching for an overt perforation if not immediately obvious, avoidance of dividing sigmoid adhesions or dissecting omentum from the sigmoid colon. Most surgeons place a non-suction drain although this is not completely necessary.

\section{Clinical failure of laparoscopic lavage}

Failure of laparoscopic lavage failure can be indicated by lack of clinical improvement, worsening sepsis, development of peritonitis and requirement for re-intervention. The most common reasons for failure of laparoscopic lavage are likely incorrect selection of patients (Hinchey IV) as well as inappropriate management of overt colonic perforation (perhaps attempted primary closure rather than progressing to resection). There is a cohort of patients who develop an intra-abdominal collection post-operatively that can be treated with radiological guidance rather than re-operation.

\section{Evidence for laparoscopic lavage in the management of acute diverticulitis}

From a patient perspective, a minimally invasive procedure with avoidance of a stoma is obviously a favorable outcome in the context an emergency presentation with intraabdominal sepsis. Benefits of such an approach include shorter hospital and ICU stay, avoidance of a stoma and avoidance of a colonic resection. \{Rogers, 2012 \#491;Rossi, \#493; Toorenvliet, 2010 \#492\} Initial single center retrospective studies confirmed the feasibility of a laparoscopic technique while demonstrating promising outcomes. \{Myers, 2008 \#490\} A multicenter Dutch study of 38 patients however, suggested that laparoscopic lavage although successful in most patients, certain contra-indications must be recognized in order to avoid adverse outcomes. \{Swank, 2013 \#498\} These include evidence of overt perforation, feculent peritonitis and high ASA grade or significant co-morbidities. Considering conflicting reports and a need to validate a new minimally invasive technique, several randomized controlled trials were undertaken to evaluate the optimal management of acute perforated diverticulitis.

Three European randomized controlled trials were established to investigate the use of laparoscopic lavage in the management of acute purulent diverticulitis. Unfortunately, these trials used different study protocols, different endpoints and therefore are not directly comparable. Several systematic reviews and meta-analyses of these trials were subsequently published also producing conflicting conclusions. Therefore, over the last decade no concrete consensus has been reached regarding the use of laparoscopic lavage in diverticulitis and expert opinions still advise treating patients on a case by case basis.

DILALA The DILALA trial was designed as a prospective, randomized, controlled trial (1:1) of laparoscopic lavage versus open Hartmann procedure from Sweden and Denmark. 
Eighty-three patients were randomized (LL=39, HP=36). \{Angenete, 2016 \#496; Thornell, 2016 \#488\} There was no difference in morbidity or mortality between groups. Re-operation and re-intervention rates between the groups were similar. The LL group had shorter operating times and shorter LOS. Interestingly, patients were randomized after the diagnostic laparoscopy which helped to avoid misdiagnosis and allowed for accurate Hinchey staging. It is also important to note that the surgeons involved in this study did not receive any training. The two-year results of the DILALA trial affirm the safety and efficacy of LL. \{Kohl, $2018 \# 487\}$

LADIES-This a multicentre, parallel-group, randomised, open-label trial in 34 teaching hospitals and eight academic hospitals in Belgium, Italy, and the Netherlands. \{Vennix, 2015 \#486 \} The trial was set up with two arms- after laparoscopy, those with purulent peritonitis are treated with laparoscopic lavage and drainage, Hartmann's procedure or sigmoidectomy with primary anastomosis in a ratio of 2:1:1 (LOLA-arm), or if there is evidence of feculent peritonitis, patients will be randomised to Hartmann's procedure or resection with primary anastomosis in a ratio of 1:1 (DIVA-arm). \{Swank, $2010 \# 485\}$ This trial was terminated prematurely due to safety concerns at ineterim analysis. There was a higher complication rate in the LL arm compared to the resection arm (39\% vs 19\%) although drainage of abscess was included as an intervention. In addition, surgical reintervention was required for feculent peritonitis and overt perforation in $6 / 46$ patients suggesting that this had been missed at the index laparoscopy. One of the issues with this study is that the surgical technique was not standardised and there is no mention as to the experience and skill of the operating surgeons. The study protocol advised gentle mobilisation of the sigmoid colon but this is likely too vague a description for a surgical technique. Also, the numbers per institution is small; 46 patients were recruited from 28 different hospitals. There is no breakdown as to how many individual surgeons were involved and what their surgical background is (colorectal specialist vs emergency surgeon vs trainee). Ultimately, $76 \%$ of patients undergoing LL were treated successfully and were stoma free in the short term. Perhaps, if there had been a pre-trial training protocol for surgeons then missed perforations would not have been an issue during the trial.

SCANDIV—SCANDIV was designed as a two- armed, open- labelled, randomized, clinical, pragmatic, superiority, multicenter study based in Sweden and Norway. \{Schultz, 2017 \#484;Schultz, 2015 \#497\} Hartmann's procedure was performed in all patients with fecal peritonitis (Hinchey grade IV), including those with a visible defect in the colon wall. Randomization occurred pre-operatively rather than based on intra-operative findings. There were 101 patients in the LL group and 98 in the colonic resection (CR) group. The primary outcome was severe postoperative complications within 90 days. The study found no significant difference between the groups in this regard (30.7\% (LL) vs $26 \%$ (CR)). Mortality did not significantly differ between the groups either. There was a higher rate of intra-abdominal abscess in the LL group as well as an increased rate of re-operation. Overall, there was a $15 \%$ rate of stoma formation in the LL group vs $75 \%$ in the CR group.

LLO study-This was a retrospective multicenter international study included consecutive patients from 24 centres who underwent laparoscopic lavage from 2005 to 2015. \{Binda, 
2018 \#495\} Although this study has limitations in view of the fact it is retrospective, it includes the largest number of patients. In this study, up to $14 \%$ of patients required further surgery. Of the 231 patients treated with LL, $75 \%$ were treated successfully. Factors associated with failure of LL were higher values of MPI (peritonitis score), a high ASA grade, identification of a free perforation and extensive adhesiolysis.

In an effort to collate the evidence from the aforementioned trials, several meta-analyses and systematic reviews have been conducted. \{Acuna, 2018 \#87;Angenete, 2017 \#480;Binda, 2018 \#495;Cirocchi, 2019 \#318;Marshall, 2017 \#481;Penna, 2018 \#483\} Interestingly, although the same studies were included in the analysis by these different groups, the conclusions are not the same. Overall, there is no difference in mortality between patients undergoing laparoscopic lavage and some form of colonic resection (most commonly Hartman's procedure). It is evident that laparoscopic lavage has a higher complication rate and a surgical re-intervention rate of approximately 15\%-20\% across the studies. Despite the higher incidence of re-intervention, this does not equate to adverse outcomes in this subgroup. On the contrary, there is a 75\% success rate with laparoscopic lavage as an index procedure with a stoma formation rate of only $15 \%$. The merits of laparoscopic lavage are very much dependent on the surgeon and patient outlook. A re-intervention may appear to be a failure of the technique. However, one could view laparoscopic lavage as an initial temporizing surgical option with the chance that a patient can avoid a stoma. If this treatment fails, then a surgical resection is required.

Long Term Outcomes-Laparoscopic lavage was originally instigated as an emergency procedure to deal with acute intra-abdominal sepsis in a minimally invasive manner. The long term outcomes are secondary endpoints that don't hold much clinical relevance to the patient with peritonitis. However, at 12 month follow up, patients undergoing laparoscopic lavage have a reduced need for operative intervention and a lower rate of stoma formation compared to those undergoing a colonic resection. \{Angenete, $2017 \# 480$ \}

\section{Cost Analysis}

A health economic analysis of the DILALA trial demonstrated a significant cost reduction in the laparoscopic lavage group compared to the patients undergoing a Hartman's procedure. \{Gehrman, 2016 \#478\} Vennix et al conducted a cost-utility analysis as part of the Ladies trial, reporting outcomes in quality adjusted life years (QALYs) however, no difference was shown for either primary clinical outcome or QALYs between groups. \{Vennix, $2015 \# 486$ \}

\section{Predictors of failure of laparoscopic lavage}

Ideally, laparoscopic lavage is successful when it can be completed technically with control of sepsis obviating the need for re-intervention or a stoma. Efforts have been made to identify factors predictive of failure of this technique. Patient factors include high ASA grade \{Liang, $2012 \# 477$ \} and use of immunosuppressants. \{Greilsamer, 2017 \#476\} Surgical factors such as feculent peritonitis or overt colonic perforation should be a contraindication to laparoscopic lavage and should prompt definitive resection. 


\section{Conclusion}

It is important to focus on the clinical reasoning behind the development of this technique in order to consider and evaluate its success. When faced in an emergency situation with intraabdominal sepsis patients would prefer the least invasive but safest option. It could be suggested that the longer term outcomes at 3 months and even up to 2 years following the index event are not so relevant at a point in time of life threatening disease. When put into perspective, if laparoscopic lavage can ideally treat but otherwise temporize intra-abdominal sepsis from diverticulitis it most certainly has a role.

\section{Segmental Colitis Associated with Diverticulitis versus Diverticulitis - How to Tell the Difference?}

Segmental colitis associated with diverticulitis (SCAD) is an inflammatory condition affecting the colon in segments that are also affected by diverticulosis, namely, the sigmoid colon. While SCAD is considered a separate clinical entity, it is frequently confused with diverticulitis or inflammatory bowel disease (IBD). SCAD affects approximately $1.4 \%$ of the general population and 1.15 to $11.4 \%$ of those with diverticulosis and most commonly affects those in their $6^{\text {th }}$ decade of life. $\{$ Imperiali, 2000 \#668;Tursi, 2011 \#667\} The exact pathogenesis of SCAD is unknown, but proposed mechanisms include mucosal redundancy and prolapse occurring in diverticular segments, fecal stasis, and localized ischemia. \{Tursi, $2011 \# 669$ \} The histopathology of SCAD has been compared to that of ulcerative colitis (UC), with both sharing features such as an inflammatory infiltrate, cytoarchitectural distortion, crypt abscess formation. \{Lamps, 2007 \#670\} Less commonly, histopathologic findings can mimic Crohn's disease (CD). A mechanism of bacterial stasis within the colon segment affected by diverticulosis has been suggested similar to fecal stasis occurring in the rectum in ulcerative colitis or terminal ileum in CD. \{Shepherd, $1996 \# 671$ \}

Since SCAD always involves a segment of colon with diverticulosis, distinguishing this less common condition from diverticulitis can be challenging. Astute attention to the unique clinical, endoscopic, and histopathologic findings in SCAD can help make the correct diagnosis. Clinically, SCAD can present with rectal bleeding, diarrhea, or abdominal pain and up to one third of patients will have more than one symptom at the time of diagnosis. However, systemic features such as fever, leukocytosis and weight loss are rare. \{Harpaz, 2006 \#672\} Diverticulitis, on the other hand, frequently features abdominal pain, with severe cases presenting with fever and leukocytosis, while hematochezia and diarrhea are rare. Laboratory tests (complete blood count, C-reactive protein, erythrocyte sedimentation rate, fecal calprotectin and stool bacterial, ova, and parasite tests) and cross-sectional imaging are generally non-specific but may be ordered to help rule out other diagnoses such as infections, ischemic colitis and malignancy. Further, since SCAD generally affects older individuals, those who are young are less likely to fit the clinical profile.

Endoscopy is the mainstay of SCAD diagnosis. \{Tursi, 2010 \#673\} The key feature of SCAD that helps distinguish it from diverticulitis is sparing of diverticular orifices. On the contrary, in diverticulitis, the typical endoscopic feature is either complete resolution of inflammation or inflammation surrounding the diverticular orifice. Endoscopically, SCAD 
can appear similar to IBD, however, limited to the diverticular segment of colon. Four subtypes of SCAD have been established based on endoscopic features. Type A, crescentic fold disease, affects the mucosal colonic folds but spares the diverticular orifice.

Histopathology shows active inflammation with neutrophil and lymphocyte infiltrate limited to the crypt epithelium without crypt architectural distortion. Type B, mild-to-moderate UClike disease, has endoscopic features similar to UC with diffuse loss of vascular pattern, edema, hyperemia and erosions. Histopathologic features include chronic inflammation and crypt architectural distortion similar to UC. Unlike UC however, the rectum is spared. Type $\mathrm{C}, \mathrm{CD}$-like disease features isolated aphthous ulcers endoscopically and transmucosal inflammation and microfissures on histopathologic evaluation. Type D, severe UC-like disease is similar to type B but more severe with diffuse ulceration with histologic findings of crypt abscess and goblet cell depletion. Unlike UC, SCAD features rectal sparing on initial endoscopy. The remainder of the gastrointestinal tract should be evaluated to rule out other areas of disease suggesting CD.

Most case of SCAD resolve with a high-fiber diet and antibiotics, with salicylates reserved for more severe cases. Relapse is uncommon and immunosuppression with steroids is rarely needed. A relapsing clinical course may suggest a diagnosis of IBD and treatment as such should be initiated. Surgery is extremely uncommon and reserved for severe refractory disease.

\section{Unusual Location of Diverticular Disease}

\section{Abstract}

Diverticular disease is a frequent disease affecting nearly $50 \%$ of adults over 50 years old in Western countries. The sigmoid colon involvement is considered the most common.

However, in the Asian countries, right-sided diverticulitis outnumbers the left. This difference seems to be secondary to dietary and genetic factors. Differential diagnosis might be difficult because of similarity with appendicitis. However accurate imaging studies allow a precise preoperative diagnosis and management planning. Transverse colonic diverticulitis is very rare accounting for less than $1 \%$ of colonic diverticulitis with a perforation rate that has been estimated to be even more rare. Rectal diverticula are mostly asymptomatic and diagnosed incidentally in the majority of patients and rarely require treatment. Giant colonic diverticula (GCD) is a rare presentation of diverticular disease of the colon and it is defined as an air-filled cystic diverticulum larger than $4 \mathrm{~cm}$ in diameter. The pathogenesis of GCD is not well defined even though several theories have been described.

In this article we describe the characteristic and the management of unusual locations of diverticulitis: right colonic-, transverse- and rectal-diverticulitis as well as GCD.

\section{Right Colonic Diverticulitis}

Right colonic diverticulitis (RCD) is a rare disorder in Western countries ${ }^{236}$ with a reported incidence of $1-5 \%$ in Western populations, as opposed to $76 \%$ in Asian countries. ${ }^{237}$

In particular, Kim et al ${ }^{236}$ reported that $76 \%$ of diverticulitis occurred in the right side of the colon meanwhile only $24 \%$ on the left side. This difference seems to be secondary to dietary 
and genetic factors. ${ }^{238}$ Interestingly, the location of the diverticula in the right colon is more predominant in blacks than in whites supporting the role of genetic factors. ${ }^{75}$

The RCD differ from the left-sided diverticulitis (LCD) for several aspects. First, right-sided diverticula, even though there are still some concerns regarding the origins, are usually solitary true diverticula with a congenital nature. ${ }^{239}$ Conversely, when they are multiple, i.e. acquired and false diverticula, increased intraluminal pressure and abnormal right-colon motility contribute to the pathogenesis. ${ }^{240}$ Second, patients with RCD are significantly younger and with a more benign course than patients with LCD. ${ }^{241,242}$ In fact, differently from LCD, only $20 \%$ of patients with RCD will have a second episode of diverticulitis within 5 years. ${ }^{243}$

Third, considering that most of right diverticula are located in the cecum, close to ileocecal valve, RCD is usually confused with acute appendicitis (AA) ${ }^{244}$ with a RCD/AA ratio of about $1 / 200 .{ }^{245}$ In this case a CT scan can help to rule out the diagnosis especially when a normal appendix is visible. ${ }^{246}$ Furthermore, patients with RCD are older than those affected by AA (20-40 vs $10-19$ years) ${ }^{247}$ excluding also the possibility of an appendiceal diverticulitis. ${ }^{248}$

Regarding the clinical presentation, most patients with RCD are asymptomatic and the diagnosis is rarely done before surgery. According to Cristaudo et al ${ }^{249}$ right abdominal pain is present in $84.6 \%$ of patients. The pain is similar to AA but the duration is generally longer and the location more lateral and superior to McBurney's point. ${ }^{249}$

Furthermore, nausea, vomiting and fever are less frequent in comparison to patients with AA. Moreover, the differential diagnosis should include several other conditions, such as cholecystitis, pancreatitis, gastritis, and peptic ulcer disease, that could mimic RCD. ${ }^{250}$ Patients with RCD are more likely to bleed and according to Faucheron et al ${ }^{251}$ this localization was associated with a high-risk of bleeding.

In RCD, CT has a 98\% sensitivity and specificity and has become the first diagnostic exam of choice increasing the preoperative diagnostic rate. ${ }^{252}$ In case of suspicious indistinguishable mass, barium enema and colonoscopy should be performed in order to rule out a colonic carcinoma.

Ultrasounds is a widely used imaging test for abdominal pain of the right lower quadrant and when performed by a skilled operator can reach a 91.3 sensitivity and a $99.8 \%$ specificity. 253 To date, the treatment of RCD remains controversial and no therapeutic guidelines have been established ${ }^{254}$ with the exception of caecal diverticulitis in which excisional treatment prevents the recurrence of symptoms. ${ }^{244}$

Conservative management with antibiotics has been suggested for the uncomplicated RCD. In this context, Oudenhoven et al ${ }^{255}$ retrospectively reviewed 44 patients diagnosed with RCD over an 11-year period. Forty-one over 44 patients were successfully treated conservatively while 3 patients received diverticulectomy. Recurred diverticulitis developed only in 5 patients that responded well to a further conservative management. 
These results were consistent with what had been described by several authors $256-258$ stating that recurrent RCD should be initially treated with conservative treatment.

If medical treatment fails and the inflammation is minimal a diverticulectomy is recommended. Right colectomy is strongly recommended in case of extensive inflammation, perforation or masses suspicious for cancer ${ }^{259}$ with a $5.8 \%$ malignancy rate reported by Poon et al. ${ }^{260}$ Appendiceal diverticulitis should be treated with a simple appendectomy.

Laparoscopic surgery can be advantageous also in case of complicate RCD with a longer operative time and a similar rate of postoperative complications. ${ }^{261}$

\section{Giant colonic diverticulum}

Giant colonic diverticulum (GCD) is a rare presentation of diverticular disease of the colon

${ }^{262}$, with almost 200 cases reported so far in the literature ${ }^{263}$, and it is defined as an airfilled cystic diverticulum larger than $4 \mathrm{~cm}$ in diameter. ${ }^{264}$

GCD mostly arise from the antimesenteric border of the sigmoid colon in about $90 \%$ of cases and have a diameter ranging from 4 to $9 \mathrm{~cm}^{264}$, with a record diameter of $40 \mathrm{~cm}$. ${ }^{265}$ The wall thickness ranges from of $0.1 \mathrm{~cm}$ to $2 \mathrm{~cm}$ and the communication between the GCD and the colonic wall is demonstrated in only $30 \%$ of the cases ${ }^{266}$. There is equal gender distribution and usually present after the sixth decade of life. ${ }^{238,267}$

GCD was first described in 1946 by Bonvin and Bonte 268 and later, in 1953 by Hughes and Green. ${ }^{269}$ Since the first description several names have been used to describe this condition, including giant colon cyst, giant air cyst or solitary air cyst but the term GDC was the preferred one in the scientific literature. ${ }^{270}$ Histopathological classification of GCD was first proposed by McNutt et al ${ }^{271}$ in 1988.

GCD can be divided in three distinct entities:

- $\quad$ Type 1 (22\%): pulsion or pseudo-diverticula containing remnants of the muscularis mucosa and muscularis propria;

- $\quad$ Type 2 (66\%): inflammatory diverticulum, secondary to local (mucosalsubserosal) perforation and resulting in a walled-off abscess. It is located on the anti-mesenteric border and contains reactive scar tissue without normal intestinal layers;

- $\quad$ Type $3(12 \%)$ : true diverticulum, usually located on the mesenteric border, that contains all four typical bowel layers and is in continuity with the gut lumen. It may have congenital origin and belongs to the category of digestive tract duplications, from which they differ for the communication with the colonic lumen.

Based on this classification, most of giant diverticula are acquired (88\%).

The pathogenesis of GCD is not well defined even though several theories have been described. 
- The Ball-Valve mechanism is one of the most popular theory. ${ }^{272}$ According to this mechanism the influx of air into a diverticulum through a narrowed inflamed neck, acting as a communicating ostium allowing a unidirectional influx, causes trapping of gas, pressure elevation and gradual enlargement of the diverticulum.

- The inflammatory mechanism is the most accepted theory for the giant inflammatory diverticula's origin. In this case, pseudocyst formation is secondary to a microperforation of the subserosa layer with the progressive dilation of a walled-off abscess cavity until a GCD in formed. The enlargement of the cavity can lead to a progressive destruction of the mucosal and serosal layers with fibrosis and granulation tissue proliferation.

- The infection with gas-forming bacteria is not widely accepted because of the absence of any bacterial growth in the cultures.

- The pathogenesis of true congenital diverticulum is similar to other intestinal duplications 273,274 .

The clinical presentation of GCD may be variable. In fact, approximately $10 \%$ of patients are asymptomatic with GCD discovered incidentally meanwhile some patients can present non-specific symptoms like constipation, nausea, vomiting, tenesmus, fever or anemia. Furthermore, acute and chronic presentation affect approximately one-third of patients. ${ }^{263}$ The most common symptom is abdominal pain (69\%), followed by constipation (17\%) and the presence of abdominal mass $(17 \%)^{263}$ referred as a "phantom tumor" 275.

The risk of malignant transformation is estimated around $2 \%{ }^{238}$ with some cases of adenocarcinoma and MALT lymphoma that have been reported in the literature. ${ }^{276-278}$ Complications such as bleeding, volvulus, bowel obstruction, perforation or fistula (small bowel, bladder) occur in $15 \%-35 \%$ of the cases. ${ }^{279}$

There is no gold standard diagnostic test for GCD even if CT scan seem to be the main imaging modality. A CT scan is the preferred imaging technique for confirming the diagnosis of GCD evaluating size, location, characteristic of the bowel wall as well as accompanying complications. Moreover, is considered to be useful in the differential diagnosis. ${ }^{263,280}$ Barium enema and colonoscopy are less effective than $\mathrm{CT}$ in identifying communication between the GDC and the large bowel lumen ${ }^{270}$ but they may show a coexistent diverticular disease and help to rule out a possible carcinoma. Barium enema should be avoided in case of risk of perforation.

Diverticulectomy or partial colectomy of the involved colonic segment, including the diverticulum, with primary anastomosis is the gold standard treatment for uncomplicated GCD. ${ }^{280}$ Unfortunately, diverticulectomy alone should be avoided due to the high-risk of dehiscence as well as for the possibility of recurrence of GCD ${ }^{264}$. In complicated cases, a two-stage resection with a mucosal fistula with terminal colostomy might be necessary. ${ }^{280}$ Conservative treatment is recommended in asymptomatic high-risk elderly patients ${ }^{281}$ and percutaneous drainage should be considered in case of well localized abscess. ${ }^{282}$ 


\section{Transverse colonic diverticulitis}

Transverse colonic diverticula represent only $6 \%$ of all gastrointestinal tract diverticula 75 and they are usually detected as asymptomatic findings on endoscopic examination. The pathogenesis of these diverticula is multifactorial and they can be true or false diverticula. Transverse colonic diverticulitis (TCD) is very rare accounting for less than $1 \%$ of colonic diverticulitis ${ }^{283}$ with a perforation rate that has been estimated to be even more rare. ${ }^{284}$

Thompson and Fox in 1944 were the first to publish a case of perforated solitary diverticulum of the transverse colon. ${ }^{285}$ Rao et al. ${ }^{286}$ described the first case of spontaneous transverse colocutaneous fistula. Patients with TCD are 15-20 years younger than patients with LCD with a predominance of females (85\%). ${ }^{287-289}$

Symptoms, diagnosis and treatment are similar of those described for RCD. In fact, in one of the most recently published case-series considering 4 patients with TCD the diagnosis was made preoperatively with a CT scan that allowed a conservative treatment. Only one patient developed sigmoid diverticulitis after the treatment. ${ }^{283}$

\section{Rectal Diverticulitis}

Rectal diverticula are more rare than other colonic diverticula with an estimated incidence of less than $0.08 \%$ of the cases. ${ }^{290,291}$ The first cases of rectal diverticulitis were described in the early part of the $20^{\text {th }}$ century by Giffin. ${ }^{292}$ After that, Spriggs and Marxer ${ }^{293}$ and Wallstad and Sahibzada ${ }^{294}$ reported 4 cases of rectal diverticulitis among 166 and 192 patients, respectively, with colonic diverticulosis establishing the highest incidence reported so far $(2 \%)$.

Rectal diverticula are typically true diverticula (Figure 2), as opposed to the colonic diverticula, suggesting the possibility that they arise at points of focal weakness located along the lateral walls of the rectum due to congenital or acquired causes. ${ }^{294}$ In fact, the complete longitudinal layer of the rectum is thinner on the lateral wall if compared with the anterior and posterior side. ${ }^{295}$

Recently, rectal diverticula after stapled transanal rectal resection have been described. 296 Even if they are usually isolated ${ }^{290}$ the most frequent number per patient ranges from one to three with a diameter $>2 \mathrm{~cm}$ or more, significantly larger than sigmoid diverticula, which are 0.5 to $1 \mathrm{~cm}^{295}$, and their size may vary with changes in intrabdominal pressure. ${ }^{297}$

The origin of rectal diverticula is unknown but there are several predisposing factors that may be linked with the disease. These factors include congenital anomalies (i.e. weakness of the rectal wall), absence of supporting structure such as the coccyx, muscle wall atrophy 291,294 and other acquired causes such as weakness of the rectovaginal septum, rectal trauma or infections, constipation or recurrent faecal impaction which causes an intraluminal pressure increase of the rectum with a subsequent dilatation. 290,298

Two theories to explain the pathogenesis of rectal diverticula have been proposed. According to the first theory the anatomical disposition of the muscle fibers of the taenia coli, which surround the rectum, may promote a major resistance to intraluminal pressure changes. ${ }^{299}$ 
The second theory suggests that the less intense rectal pressure and lesser peristaltic activity, compared with the sigmoid colon, can explain the low incidence of rectal diverticula. 298,300

Rectal Diverticula are asymptomatic and diagnosed incidentally in the majority of patients and do not require treatment. Complications associated with rectal diverticula may include diverticulitis with rectal strictures, perforation, abscess or rectovaginal fistulas. ${ }^{290,301}$ Furthermore, rectal diverticulitis can also occur due to fecal impaction and can be clinically confused with rectal carcinoma. ${ }^{299}$ In case of complications, surgical treatment is necessary and should include, depending on the severity and the extension of the disease, local drainage, diverting colostomy, diverticulectomy or abdominoperineal amputation. 290,292

\section{Conclusion}

While we have some far in our understanding of diverticular disease, much remains to be discovered. The progression from normal sigmoid colon to diverticula to diverticulitis is poorly understood. Likewise, appropriate patient selection for surgical intervention for those with recurrent diverticulitis is a work in progress. We look forward to an update of this manuscript in a decade's time and hope that we will be in a better place to help those with this disease.

\section{Acknowledgement}

The authors would like to thank Ms. Molly McDade for her artwork used in the figures of this manuscript.

Support: Dr. Hawkins' work on this manuscript was supported by the National Institute of Diabetes and Digestive and Kidney Disease of the National Institutes of Health under award number K23DK118192.

\section{Biographical Information}

Alexander T. Hawkins MD, MPH is currently an Assistant Professor of Surgery and Director of the Colorectal Research Center at the Vanderbilt University Medical Center in Nashville, TN. Dr. Hawkins received his medical degree from the University of Virginia and completed his general surgery training at the Massachusetts General Hospital in Boston, MA. He went on to serve a fellowship in Colon and Rectal Surgery at Barnes-Jewish Hospital in St Louis, MO. He is an NIH funded investigator who uses mixed-methods and patient reported outcomes to study the role of surgery in recurrent diverticulitis. His clinical focus is broad and includes colorectal cancer, inflammatory bowel disease and ano-rectal issues.

Paul E. Wise, MD is Professor of Surgery at Washington University in St. Louis School of Medicine where he currently serves as Director of the General Surgery Residency Program. He completed general surgery residency at Vanderbilt University in 2003 after completing medical school at Johns Hopkins University School of Medicine in 1996. He completed his colon and rectal surgery fellowship at Washington University/Barnes Jewish Hospital thereafter and maintains interests in hereditary colorectal cancer syndromes and surgical education. 
Tiffany Chan, MD, MHSc, is a colorectal surgeon affiliated with the University of British Columbia in Canada. She studied medicine at McMaster University in Hamilton, Ontario, Canada and completed residency in general surgery and her Masters of Health Sciences through the University of British Columbia. She completed her fellowship training in colon and rectal surgery at Washington University/Barnes Jewish Hospital in St. Louis, Missouri.

Janet T. Lee, MD, MS, FACS, FASCRS, is a clinical assistant professor at the University of Minnesota. She graduated magna cum laude from Harvard University with a BA in Anthropology. Dr. Lee completed her medical school education, general surgery residency, Master's in clinical research, and colorectal surgery fellowship at the University of Minnesota. Her current clinical and research interests include anal dysplasia, minimally invasive colorectal surgery, and pelvic floor disorders.

Tamara Glyn Mullaney, MBChB, FRACS, is a consultant colorectal surgeon and senior lecturer at the Christchurch Hospital and University of Otago, Christchurch, New Zealand. She completed fellowships in colorectal surgery at the Mayo Clinic, Rochester, MN; St Vincent's Hospital, Melbourne, Australia; and St James' Hospital, Leeds, UK. Her particular interests include inflammatory bowel disease and advanced pelvic malignancy and she is completing a Masters of Medical Science in Inflammatory Bowel Disease Associated Cancers.

Verity Wood, MBChB, FRANZCR, PGCert Travel medicine, is a consultant radiologist at Christchurch Hospital, Christchurch, New Zealand. She spent a year in Vancouver completing a Body Imaging and Intervention fellowship through the University of British Columbia. Following fellowship, she has a specialist interest in abdominal imaging and rectal cancer imaging. Verity is a member of the both the RANZCR New Zealand Branch Committee and the Safety, Quality and Standards committee.

Associate Professor Tim Eglinton, MBCHB, FRACS, MMedSc, is a Consultant Colorectal Surgeon and Head of Department of Surgery at the University of Otago, Christchurch. After obtaining FRACS in General Surgery in 2005, he undertook post fellowship training in colon and rectal surgery in Cambridge, United Kingdom and Adelaide, Australia. Tim's specialty interests include; inflammatory bowel disease, diverticular disease and colorectal cancer. He has published and lectured widely over the last decade on these and other subjects. In addition to medical student teaching, Tim has a keen interest in post graduate surgical training as a member of the RACS Surgical Skills Education and Training Committee and the ANZ Training Board in Colon and Rectal Surgery.

Professor Frank Frizelle, MBChB, MMedSc, FACS, FRACS, FASCRS, FRCSI, is a Consultant Colorectal surgeon and Professor of Surgery at Christchurch Hospital and the University of Otago, Christchurch. He completed fellowships at the Mayo Clinic, Rochester, MN and in Scotland before returning to New Zealand. He was the head of department for General Surgery for 13 years and has been editor in chief of the New Zealand Medical Journal for 18 years. His research interests include advanced pelvic cancer, inflammatory bowel disease, diverticula disease and the microbiome. 
Adil Haleem Khan, MD is a General and Bariatric surgeon at Raleigh General Hospital, WV. Dr. Khan graduated from Aga Khan university medical college in Pakistan. He did his surgical residency from Lahey Medical Center in Burlington, Massachusetts. He did fellowship in minimally invasive and bariatric surgery from University Hospitals Cleveland medical center. He currently has active General and Bariatric surgery practice in West Virginia.

Jason Hall, MD, MPH is an Associate Professor of Surgery, Co-Director of the Dempsey Center for Digestive Disorders and Chief of Colon \& Rectal Surgery at the Boston University School of Medicine. He received his medical degree from Harvard Medical School and completed his general surgery training at the Massachusetts General Hospital. He went on to the Lahey Clinic for a fellowship in Colon \& Rectal Surgery. His research interests include colorectal cancer, diverticulitis and fistulas, with several of his studies appearing in national academic journals. He is board certified by the American Board of Surgery and the American Board of Colon and Rectal Surgery and received fellowships from the American College of Surgeons and the American Society of Colon and Rectal Surgery (ASCRS). Since 2009, Hall has had an active role in the ASCRS, most recently as the chair of the awards committee.

Iyoob Ilyas, MD, FACS, FASCRS, is a colorectal and general surgeon with special interests in minimally invasive surgery including robotic surgery. He earned his medical degree from Kasturba Medical College in India and did post graduate training in Surgery from Chennai, India. He then worked and trained at University of Oxford in England, Cleveland Clinic and University of Arizona. He completed his colon and rectal fellowship and advanced robotic surgery training from Henry Ford Hospital in Michigan. He is board certified in Colorectal Surgery and General Surgery from the American College of Surgeons and American College of Colon and Rectal Surgeons. He is also a fellow of the American College of Surgeons and American Society of Colon and Rectal Surgeons. His research interest includes surgical outcomes, minimally invasive colorectal surgery particularly robotic surgery, diverticular diseases, and anorectal disease. He has multiple research publications and is also the editorial board of multiple surgical journals.

Maria Michailidou, MD, received her medical degree at University of Patras in Greece. She completed her general surgery residency at the University of Arizona and her fellowship in Colon and Rectal Surgery at Penn State Hershey Medical Center. She is board certified in General and Colorectal Surgery. She is currently a colorectal surgeon in Maine.

Valentine N. Nfonsam, MD, MS, FACS, FASCRS is a Professor of Surgery and the Interim Division Chief of Surgical Oncology at the University of Arizona. He is also the General Surgery Residency Program Director. Dr. Nfonsam graduated from the University of Illinois College of Medicine in Chicago and completed his surgery residency training at UT southwestern in Dallas and North Shore-Long Island Jewish Health System, New York. He did a research fellowship at the Cleveland Clinic, Cleveland and completed a two-year fellowship in Colon and Rectal Surgery at the University of South Florida, Tampa. Dr. Nfonsam's clinical specialties include colorectal and gastrointestinal surgery, gastrointestinal oncology and laparoscopic and roboticassisted surgery. His research focuses 
on colorectal cancer especially in the young in addition to disparities in the incidence of colorectal cancer.

Michelle Cowan, MD, is Associate Professor of Surgery and co-director of the Inflammatory Bowel Disease Program at the University of Washington. She earned her medical degree at the Medical College of Virginia (VCU) and completed a general surgery residency at the University of Chicago. She completed a fellowship in Colon and Rectal surgery at Washington University in St. Louis. She has an active clinical practice focused on inflammatory bowel disease, multidisciplinary care of rectal cancer and minimally invasive and endoscopic techniques for colon and rectal surgery.

Jennifer Williams, MD, is an Assistant Professor of Colon and Rectal Surgery at Emory University in Atlanta, Georgia. She received a Bachelor of Science degree in Biology and Psychology from Emory University and a medical degree from The David Geffen School of Medicine at UCLA. She completed a general surgery residency at Harbor-UCLA Medical Center followed by fellowship in colon and rectal surgery at The Cleveland Clinic.

Scott R. Steele, MD, MBA, is the Rupert B. Turnbull MD Endowed Chair in Colorectal Surgery and Chairman of Colorectal Surgery at Cleveland Clinic in Cleveland, OH. A graduate of the United States Military Academy at West Point, NY. He is on the editorial board for several surgical journals and serves as the Co-Editor for Diseases of the Colon \& Rectum and the Editorin-Chief for Clinics in Colon and Rectal Surgery. He also received his MBA from Case Western University Weatherhead School of Business and Management.

C. Tyler Ellis, MD, MSCR, has appointments in both the School of Medicine and School of Public Health at the University of Louisville in Louisville, KY. Dr. Ellis is both a clinical colorectal surgeon and health service researcher with research interests focus on the impact of care coordination and health care organization on the delivery of multidisciplinary care for colorectal diseases and its impact on patient outcomes.

Karim Alavi, MD, MPH is an Associate Professor in Surgery at the University of Massachusetts Medical School in Worcester, Massachusetts. He received his medical degree from the George Washington University. He completed a residency in General Surgery at the Washington Hospital Center and a fellowship in Colon \& Rectal Surgery at the University of Minnesota Medical School. His research interests include investigation of and improvement in colon and rectal surgical outcomes.

Danielle Collins, MD, FRCSI, is a consultant colorectal surgeon at Western General Hospital, Edinburgh, Scotland. She received her medical degree at Trinity College, Dublin, Ireland. Having completed general surgical training in Ireland, she underwent fellowship training at the Mayo Clinic, Rochester, MN, USA and the Royal Marsden, London, UK. Her clinical interests include robotic surgery, new surgical technology and pelvic exenteration surgery.

Des Winter, MD, FRCSI, is a consultant colorectal surgeon and clinical professor at St Vincent's University Hospital, Dublin, Ireland. Following graduation from medical school he did research spanning Ireland and USA for a doctorate by thesis before completing 
surgical training in Ireland. Des went on to fellowship training at the Mayo Clinic, Rochester, USA before returning home to his alma mater in 2006. Des is the Editor-in-Chief of BJS and has a keen interest in surgical publishing.

Karen Zaghiyan, MD, FACS, FASCRS is a board-certified colorectal surgeon in Los Angeles, California and Assistant Professor of Surgery at Cedars Sinai Medical Center. Dr. Zaghiyan graduated magna cum laude with a Bachelor of Science in Biology from the University of California, Los Angeles. She then completed medical school at the University of California, San Diego before pursuing her general surgery residency and colon and rectal surgery fellowship at Cedars-Sinai Medical Center in Los Angeles where she remained to establish her practice. Dr. Zaghiyan's research interests include rectal cancer and inflammatory bowel disease including methods to improve outcomes in patients with colon $\&$ rectal diseases. Dr. Zaghiyan is an innovator and expert in her field. She is the principal investigator of numerous local, national and international research studies and has authored numerous publications in peer reviewed journals such as Annals of Surgery and Diseases of the Colon and Rectum.

Gaetano Gallo MD, is a researcher in the Department of Medical and Surgical Sciences at University of Catanzaro, Italy. He completed his training in General Surgery at University of Catanzaro and at Department of Colorectal Surgery, S. Rita Clinic (Vercelli). Subsequently, he completed his research fellowship in colorectal surgery at Department of Surgery Academic Medical Center (AMC) (Amsterdam - The Netherland) and at Department of Digestive and Endocrine Surgery - Pole Hepato-Digestif, Nouvel Hopital Civil - University Hospital of Strasbourg. He has a clinical and academic interest in Proctology (Haemorrhoidal Disease, Perianal and Rectovaginal Fistulas, Rectal Prolapse), Functional and Pelvic Floor Disorders, IBD and Microbiome and Colorectal Cancer. He is a steering committee member of the Italian Society of Colorectal Surgery (SICCR) and is currently a member of the European Society of Coloproctology (ESCP) Guidelines Committee. He is member of the Junior Editorial Advisory Board of Colorectal Disease as well as reviewer for several colorectal journals.

Michele Carvello, MD, is a consultant surgeon in the Division of Colon and Rectal Surgery at Humanitas Clinical and Research Hospital in Milan (Italy). He completed his training in Gastrointestinal Surgery in San Raffaele Hospital in Milan (Italy). Subsequently, he completed his research fellowships at Massachusetts General Hospital (Boston, US) and at University Medical Center Utrecht (The Netherlands) focusing on minimally invasive surgery. He has a special interest in the surgical management of inflammatory bowel disease and colorectal cancer. His research interests focus mainly on inflammatory bowel disease, colorectal cancer and the application of new technologies such as fluorescence angiography.

Antonino Spinelli, MD, PhD, FASCRS, leads the Unit of Colon and Rectal Surgery of Humanitas Research Hospital, Rozzano, Milan, Italy, and is a Full Professor of Surgery at Humanitas University. He is dedicated to the development of innovative techniques in minimally invasive colorectal surgery. He is an Associate Editor for Colorectal Disease, Diseases of the Colon and Rectum, JARC, and is an Editorial Board Member for the Journal of Crohn's Colitis and for Coloproctology. He is a member of the Scientific Programme 
Committee of ESCP, Assistant Secretary of ESCP, Member of IOIBD and of ISFGS. He is an honorary Fellow of the Czech Society of Surgery and Honorary Member of the Brazilian College of Digestive Surgery.

Amy L. Lightner, MD is an Associate Professor of Surgery in the Department of Colon and Rectal Surgery at Cleveland Clinic, OH, the Associate chair of Surgical Research, and the Primary Investigator of the Surgical Inflammatory Bowel Disease Translational Laboratory in the Lerner Research Institute where she is an Associate Professor of Inflammation and Immunity. She completed her surgical training in General Surgery at University of California at Los Angeles, post-doctoral work at Stanford University under a California Institute of Regenerative Medicine Training Grant focused on the differentiation pathways of embryonic stem cells and a fellowship at the Mayo Clinic. She is the primary investigator of a multi-center international randomized control trial studying a variation in surgical technique for ileocolic Crohn's disease and the primary investigator for an industry sponsored trial for stem cell therapy in Crohn's disease.

\section{REFERENCES}

1. Peery AF, Barrett PR, Park D, et al. A high-fiber diet does not protect against asymptomatic diverticulosis. Gastroenterology. 2012;142(2):266-272 e261. [PubMed: 22062360]

2. Shahedi K, Fuller G, Bolus R, et al. Long-term risk of acute diverticulitis among patients with incidental diverticulosis found during colonoscopy. Clin Gastroenterol Hepatol. 2013;11(12):16091613. [PubMed: 23856358]

3. Stollman N, Smalley W, Hirano I, Committee AGAICG. American Gastroenterological Association Institute Guideline on the Management of Acute Diverticulitis. Gastroenterology. 2015;149(7):1944-1949. [PubMed: 26453777]

4. Strate LL, Modi R, Cohen E, Spiegel BM. Diverticular disease as a chronic illness: evolving epidemiologic and clinical insights. Am J Gastroenterol. 2012;107(10):1486-1493. [PubMed: 22777341]

5. Humes DJ, Solaymani-Dodaran M, Fleming KM, Simpson J, Spiller RC, West J. A populationbased study of perforated diverticular disease incidence and associated mortality. Gastroenterology. 2009;136(4):1198-1205. [PubMed: 19185583]

6. Everhart JE, Ruhl CE. Burden of digestive diseases in the United States Part III: Liver, biliary tract, and pancreas. Gastroenterology. 2009;136(4):1134-1144. [PubMed: 19245868]

7. Sandler RS, Everhart JE, Donowitz M, et al. The burden of selected digestive diseases in the United States. Gastroenterology. 2002;122(5):1500-1511. [PubMed: 11984534]

8. Etzioni DA, Mack TM, Beart RW, Kaiser AM. Diverticulitis in the United States: 1998-2005: changing patterns of disease and treatment. Ann Surg. 2009;249(2):210-217. [PubMed: 19212172]

9. Painter NS. Diverticular disease of the colon--a disease of the century. Lancet. 1969;2(7620):586588. [PubMed: 4186011]

10. Painter NS, Burkitt DP. Diverticular disease of the colon: a deficiency disease of Western civilization. Br Med J. 1971;2(5759):450-454. [PubMed: 4930390]

11. Painter NS, Truelove SC, Ardran GM, Tuckey M. Segmentation and the Localization of Intraluminal Pressures in the Human Colon, with Special Reference to the Pathogenesis of Colonic Diverticula. Gastroenterology. 1965;49:169-177. [PubMed: 14323727]

12. Burkitt DP, Walker AR, Painter NS. Effect of dietary fibre on stools and the transit-times, and its role in the causation of disease. Lancet. 1972;2(7792):1408-1412. [PubMed: 4118696]

13. Mayo WJWL, Giffin HZ. . Acquired diverticulitis of the large intestine. . Surg Gynecol Obstet 1907:58-115.

14. Mayo WJ. Diverticula of the Sigmoid. Ann Surg. 1930;92(4):739-743. [PubMed: 17866413]

15. Rankin FW BP. Diverticulitis of the colon. . Surg Gynecol Obstet 1930;30:836-847. 
16. Sanderson ER. Henri Hartmann and the Hartmann operation. Arch Surg. 1980;115(6):792-793. [PubMed: 6992738]

17. Parks TG. Natural history of diverticular disease of the colon. A review of 521 cases. Br Med J. 1969;4(5684):639-642. [PubMed: 5359917]

18. Roberts P, Abel M, Rosen L, et al. Practice parameters for sigmoid diverticulitis. The Standards Task Force American Society of Colon and Rectal Surgeons. Dis Colon Rectum. 1995;38(2):125132. [PubMed: 7851165]

19. Kohler L, Sauerland S, Neugebauer E. Diagnosis and treatment of diverticular disease: results of a consensus development conference. The Scientific Committee of the European Association for Endoscopic Surgery. Surg Endosc. 1999;13(4):430-436. [PubMed: 10094765]

20. Anaya DA, Flum DR. Risk of emergency colectomy and colostomy in patients with diverticular disease. Arch Surg. 2005;140(7):681-685. [PubMed: 16027334]

21. Chapman JR, Dozois EJ, Wolff BG, Gullerud RE, Larson DR. Diverticulitis: a progressive disease? Do multiple recurrences predict less favorable outcomes? Ann Surg. 2006;243(6):876-830; discussion 880-873. [PubMed: 16772791]

22. Dharmarajan S, Hunt SR, Birnbaum EH, Fleshman JW, Mutch MG. The efficacy of nonoperative management of acute complicated diverticulitis. Dis Colon Rectum. 2011;54(6):663-671. [PubMed: 21552049]

23. Ambrosetti P, Becker C, Terrier F. Colonic diverticulitis: impact of imaging on surgical management -- a prospective study of 542 patients. Eur Radiol. 2002;12(5):1145-1149. [PubMed: 11976860]

24. Kaiser AM, Jiang JK, Lake JP, et al. The management of complicated diverticulitis and the role of computed tomography. Am J Gastroenterol. 2005;100(4):910-917. [PubMed: 15784040]

25. Hjern F, Josephson T, Altman D, Holmstrom B, Johansson C. Outcome of younger patients with acute diverticulitis. Br J Surg. 2008;95(6):758-764. [PubMed: 18412297]

26. Guzzo J, Hyman N. Diverticulitis in young patients: is resection after a single attack always warranted? Dis Colon Rectum. 2004;47(7):1187-1190; discussion 1190-1181. [PubMed: 15148645]

27. Andeweg CS, Groenewoud J, van der Wilt GJ, van Goor H, Bleichrodt RP. A Markov Decision Model to Guide Treatment of Recurrent Colonic Diverticulitis. Clin Gastroenterol Hepatol. 2016;14(1):87-95 e82. [PubMed: 25766651]

28. Salem L, Veenstra DL, Sullivan SD, Flum DR. The timing of elective colectomy in diverticulitis: a decision analysis. J Am Coll Surg. 2004;199(6):904-912. [PubMed: 15555974]

29. Janes S, Meagher A, Frizelle FA. Elective surgery after acute diverticulitis. Br J Surg. 2005;92(2):133-142. [PubMed: 15685694]

30. Feingold D, Steele SR, Lee S, et al. Practice parameters for the treatment of sigmoid diverticulitis. Dis Colon Rectum. 2014;57(3):284-294. [PubMed: 24509449]

31. 2015-2020 DGfA. Nutritional Goals for Age-Sex Groups Based on Dietary Reference Intakes and Dietary Guidelines Recommendations. https://health.gov/dietaryguidelines/2015/guidelines/ appendix-7. Accessed July 10, 2019.

32. Crowe FL, Balkwill A, Cairns BJ, et al. Source of dietary fibre and diverticular disease incidence: a prospective study of UK women. Gut. 2014;63(9):1450-1456. [PubMed: 24385599]

33. Strate LL, Liu YL, Syngal S, Aldoori WH, Giovannucci EL. Nut, corn, and popcorn consumption and the incidence of diverticular disease. JAMA. 2008;300(8):907-914. [PubMed: 18728264]

34. Peery AF, Sandler RS, Ahnen DJ, et al. Constipation and a low-fiber diet are not associated with diverticulosis. Clin Gastroenterol Hepatol. 2013;11(12):1622-1627. [PubMed: 23891924]

35. Carabotti M, Annibale B, Severi C, Lahner E. Role of Fiber in Symptomatic Uncomplicated Diverticular Disease: A Systematic Review. Nutrients. 2017;9(2).

36. Crowe FL, Appleby PN, Allen NE, Key TJ. Diet and risk of diverticular disease in Oxford cohort of European Prospective Investigation into Cancer and Nutrition (EPIC): prospective study of British vegetarians and non-vegetarians. BMJ. 2011;343:d4131. [PubMed: 21771850]

37. Horner JL. Natural history of diverticulosis of the colon. Am J Dig Dis. 1958;3(5):343-350. [PubMed: 13520671] 
38. Tarleton S, DiBaise JK. Low-residue diet in diverticular disease: putting an end to a myth. Nutr Clin Pract. 2011;26(2):137-142. [PubMed: 21447765]

39. Aldoori WH, Giovannucci EL, Rockett HR, Sampson L, Rimm EB, Willett WC. A prospective study of dietary fiber types and symptomatic diverticular disease in men. J Nutr. 1998;128(4):714719. [PubMed: 9521633]

40. Vanhauwaert E, Matthys C, Verdonck L, De Preter V. Low-residue and low-fiber diets in gastrointestinal disease management. Adv Nutr. 2015;6(6):820-827. [PubMed: 26567203]

41. Lijoi D, Ferrero S, Mistrangelo E, et al. Bowel preparation before laparoscopic gynaecological surgery in benign conditions using a 1-week low fibre diet: a surgeon blind, randomized and controlled trial. Arch Gynecol Obstet. 2009;280(5):713-718. [PubMed: 19229545]

42. Woolner JT, Kirby GA. Clinical audit of the effects of low-fibre diet on irritable bowel syndrome. Journal of Human Nutrition and Dietetics. 2000;13(4):249-253.

43. Dahl C, Crichton M, Jenkins J, et al. Evidence for Dietary Fibre Modification in the Recovery and Prevention of Reoccurrence of Acute, Uncomplicated Diverticulitis: A Systematic Literature Review. Nutrients. 2018;10(2).

44. Peery AF, Crockett SD, Barritt AS, et al. Burden of Gastrointestinal, Liver, and Pancreatic Diseases in the United States. Gastroenterology. 2015;149(7):1731-1741.e1733. [PubMed: 26327134]

45. Warner E, Crighton EJ, Moineddin R, Mamdani M, Upshur R. Fourteen-year study of hospital admissions for diverticular disease in Ontario. Can J Gastroenterol. 2007;21(2):97-99. [PubMed: 17299613]

46. Hughes LE. Postmortem survey of diverticular disease of the colon. II. The muscular abnormality of the sigmoid colon. Gut. 1969;10(5):344-351. [PubMed: 5771666]

47. Peery AF, Crockett SD, Murphy CC, et al. Burden and Cost of Gastrointestinal, Liver, and Pancreatic Diseases in the United States: Update 2018. Gastroenterology. 2019;156(1):254272.e211. [PubMed: 30315778]

48. Lee YS. Diverticular disease of the large bowel in Singapore. An autopsy survey. Dis Colon Rectum. 1986;29(5):330-335. [PubMed: 3084185]

49. Walker AR, Segal I. Epidemiology of noninfective intestinal diseases in various ethnic groups in South Africa. Isr J Med Sci. 1979;15(4):309-313. [PubMed: 447496]

50. Wheat CL, Strate LL. Trends in Hospitalization for Diverticulitis and Diverticular Bleeding in the United States From 2000 to 2010. Clin Gastroenterol Hepatol. 2016;14(1):96-103.e101. [PubMed: 25862988]

51. Stemmermann GN. Patterns of disease among Japanese living in Hawaii. Arch Environ Health. 1970;20(2):266-273. [PubMed: 5411399]

52. Lee TH, Setty PT, Parthasarathy G, et al. Aging, Obesity, and the Incidence of Diverticulitis: A Population-Based Study. Mayo Clin Proc. 2018;93(9):1256-1265. [PubMed: 30193674]

53. Bharucha AE, Parthasarathy G, Ditah I, et al. Temporal Trends in the Incidence and Natural History of Diverticulitis: A Population-Based Study. Am J Gastroenterol. 2015;110(11):15891596. [PubMed: 26416187]

54. Granlund J, Svensson T, Olén O, et al. The genetic influence on diverticular disease--a twin study. Aliment Pharmacol Ther. 2012;35(9):1103-1107. [PubMed: 22432696]

55. Strate LL, Erichsen R, Baron JA, et al. Heritability and familial aggregation of diverticular disease: a population-based study of twins and siblings. Gastroenterology. 2013;144(4):736-742.e731; quiz e714. [PubMed: 23313967]

56. Hall JF, Roberts PL, Ricciardi R, et al. Long-term follow-up after an initial episode of diverticulitis: what are the predictors of recurrence? Dis Colon Rectum. 2011;54(3):283-288. [PubMed: 21304297]

57. Freeman HJ. Segmental colitis associated diverticulosis syndrome. World J Gastroenterol. 2016;22(36):8067-8069. [PubMed: 27688648]

58. Strate LL, Morris AM. Epidemiology, Pathophysiology, and Treatment of Diverticulitis. Gastroenterology. 2019;156(5):1282-1298.e1281. [PubMed: 30660732]

59. Freeman HJ. Natural history and long-term clinical behavior of segmental colitis associated with diverticulosis (SCAD syndrome). Dig Dis Sci. 2008;53(9):2452-2457. [PubMed: 18338265] 
60. Bassotti G, Battaglia E, Spinozzi F, Pelli MA, Tonini M. Twenty-four hour recordings of colonic motility in patients with diverticular disease: evidence for abnormal motility and propulsive activity. Dis Colon Rectum. 2001;44(12):1814-1820. [PubMed: 11742167]

61. Hinchey EJ, Schaal PG, Richards GK. Treatment of perforated diverticular disease of the colon. Adv Surg. 1978;12:85-109. [PubMed: 735943]

62. Wasvary H, Turfah F, Kadro O, Beauregard W. Same hospitalization resection for acute diverticulitis. Am Surg. 1999;65(7):632-635; discussion 636. [PubMed: 10399971]

63. Bogardus ST. What do we know about diverticular disease? A brief overview. J Clin Gastroenterol. 2006;40 Suppl 3:S108-111. [PubMed: 16885691]

64. Whiteway J, Morson BC. Elastosis in diverticular disease of the sigmoid colon. Gut. 1985;26(3):258-266. [PubMed: 3972272]

65. Wess L, Eastwood MA, Wess TJ, Busuttil A, Miller A. Cross linking of collagen is increased in colonic diverticulosis. Gut. 1995;37(1):91-94. [PubMed: 7672689]

66. PAINTER NS, TRUELOVE SC THE INTRALUMINAL PRESSURE PATTERNS IN DIVERTICULOSIS OF THE COLON. I. RESTING PATTERNS OF PRESSURE. II. THE EFFECT OF MORPHINE. Gut. 1964;5:201-213. [PubMed: 14178701]

67. Humes DJ, West J. Role of acute diverticulitis in the development of complicated colonic diverticular disease and 1-year mortality after diagnosis in the UK: population-based cohort study. Gut. 2012;61(1):95-100. [PubMed: 21551188]

68. Daniels L, Budding AE, de Korte N, et al. Fecal microbiome analysis as a diagnostic test for diverticulitis. Eur J Clin Microbiol Infect Dis. 2014;33(11):1927-1936. [PubMed: 24894339]

69. Frank DN, St Amand AL, Feldman RA, Boedeker EC, Harpaz N, Pace NR. Molecularphylogenetic characterization of microbial community imbalances in human inflammatory bowel diseases. Proc Natl Acad Sci U S A. 2007;104(34):13780-13785. [PubMed: 17699621]

70. Gevers D, Kugathasan S, Denson LA, et al. The treatment-naive microbiome in new-onset Crohn's disease. Cell Host Microbe. 2014;15(3):382-392. [PubMed: 24629344]

71. Gueimonde M, Ouwehand A, Huhtinen H, Salminen E, Salminen S. Qualitative and quantitative analyses of the bifidobacterial microbiota in the colonic mucosa of patients with colorectal cancer, diverticulitis and inflammatory bowel disease. World J Gastroenterol. 2007;13(29):3985-3989. [PubMed: 17663515]

72. Schafmayer C, Harrison JW, Buch S, et al. Genome-wide association analysis of diverticular disease points towards neuromuscular, connective tissue and epithelial pathomechanisms. Gut. 2019;68(5):854-865. [PubMed: 30661054]

73. Teetor T, Palachick B, Grim R, et al. The Changing Epidemiology of Diverticulitis in the United States. Am Surg. 2017;83(4):e134-136. [PubMed: 28424119]

74. Nguyen GC, Sam J, Anand N. Epidemiological trends and geographic variation in hospital admissions for diverticulitis in the United States. World journal of gastroenterology. 2011;17(12):1600-1605. [PubMed: 21472127]

75. Peery AF, Keku TO, Martin CF, et al. Distribution and Characteristics of Colonic Diverticula in a United States Screening Population. Clin Gastroenterol Hepatol. 2016;14(7):980-985.e981. [PubMed: 26872402]

76. Loffeld RJ. Long-term follow-up and development of diverticulitis in patients diagnosed with diverticulosis of the colon. Int J Colorectal Dis. 2016;31(1):15-17. [PubMed: 26410266]

77. Bollom A, Austrie J, Hirsch W, et al. Emergency Department Burden of Diverticulitis in the USA, 2006-2013. Dig Dis Sci. 2017;62(10):2694-2703. [PubMed: 28332105]

78. Greenwood-Ericksen MB, Havens JM, Ma J, Weissman JS, Schuur JD. Trends in Hospital Admission and Surgical Procedures Following ED visits for Diverticulitis. The western journal of emergency medicine. 2016;17(4):409-417. [PubMed: 27429691]

79. Eglinton T, Nguyen T, Raniga S, Dixon L, Dobbs B, Frizelle FA. Patterns of recurrence in patients with acute diverticulitis. Br J Surg. 2010;97(6):952-957. [PubMed: 20474006]

80. Kang JY, Hoare J, Tinto A, et al. Diverticular disease of the colon--on the rise: a study of hospital admissions in England between 1989/1990 and 1999/2000. Aliment Pharmacol Ther. 2003;17(9):1189-1195. [PubMed: 12752356] 
81. Seraina F, Cuerel AP, Hahnloser D. Current practice of in-hospital management of acute colonic diverticulitis: a nation's experience. Colorectal Disease. 2015;17 3-9.

82. Ünlü Ç, van de Wall BJ, Gerhards MF, et al. Influence of Age on Clinical Outcome of Acute Diverticulitis. 2013;17(9):1651-1656.

83. O'Leary DP, Lynch N, Clancy C, Winter DC, Myers E. International, Expert-Based, Consensus Statement Regarding the Management of Acute DiverticulitisConsensus Statement Regarding the Management of Acute DiverticulitisConsensus Statement Regarding the Management of Acute Diverticulitis. JAMA Surgery. 2015;150(9):899-904. [PubMed: 26176318]

84. Laurell H, Hansson LE, Gunnarsson U. Acute diverticulitis--clinical presentation and differential diagnostics. Colorectal Dis. 2007;9(6):496-501; discussion 501-492. [PubMed: 17573742]

85. Jamal Talabani A, Endreseth BH, Lydersen S, Edna TH. Clinical diagnostic accuracy of acute colonic diverticulitis in patients admitted with acute abdominal pain, a receiver operating characteristic curve analysis. International journal of colorectal disease. 2017;32(1):41-47. [PubMed: 27613727]

86. Schreyer AG, Layer G. S2k Guidlines for Diverticular Disease and Diverticulitis: Diagnosis, Classification, and Therapy for the Radiologist. RoFo : Fortschritte auf dem Gebiete der Rontgenstrahlen und der Nuklearmedizin. 2015;187(8):676-684. [PubMed: 26019048]

87. Balthazar EJ, Megibow A, Schinella RA, Gordon R. Limitations in the CT diagnosis of acute diverticulitis: comparison of CT, contrast enema, and pathologic findings in 16 patients. AJR American journal of roentgenology. 1990;154(2):281-285. [PubMed: 2105015]

88. Destigter KK, Keating DP. Imaging update: acute colonic diverticulitis. Clinics in colon and rectal surgery. 2009;22(3):147-155. [PubMed: 20676257]

89. Caputo P, Rovagnati M, Carzaniga PL. Is it possible to limit the use of CT scanning in acute diverticular disease without compromising outcomes? A preliminary experience. Ann Ital Chir. 2015;86(1):51-55. [PubMed: 25816854]

90. Maconi G, Carmagnola S, Guzowski T. Intestinal Ultrasonography in the Diagnosis and Management of Colonic Diverticular Disease. J Clin Gastroenterol. 2016;50 Suppl 1:S20-22. [PubMed: 27622354]

91. Lameris W, van Randen A, Bipat S, Bossuyt PM, Boermeester MA, Stoker J. Graded compression ultrasonography and computed tomography in acute colonic diverticulitis: meta-analysis of test accuracy. Eur Radiol. 2008;18(11):2498-2511. [PubMed: 18523784]

92. Cho KC, Morehouse HT, Alterman DD, Thornhill BA. Sigmoid diverticulitis: diagnostic role of CT--comparison with barium enema studies. Radiology. 1990;176(1):111-115. [PubMed: 2191360]

93. Sartelli M, Moore FA, Ansaloni L, et al. A proposal for a CT driven classification of left colon acute diverticulitis. World journal of emergency surgery : WJES. 2015;10:3. [PubMed: 25972914]

94. Thorisson A, Smedh K, Torkzad MR, Pahlman L, Chabok A. CT imaging for prediction of complications and recurrence in acute uncomplicated diverticulitis. Int J Colorectal Dis. 2016;31(2):451-457. [PubMed: 26490053]

95. Ambrosetti P, Grossholz M, Becker C, Terrier F, Morel P. Computed tomography in acute left colonic diverticulitis. Br J Surg. 1997;84(4):532-534. [PubMed: 9112910]

96. Shin S, Kim D, Kang UR, Yang C-S. Impact of CT imaging on predicting the surgical management of acute diverticulitis. Annals of surgical treatment and research. 2018;94(6):322-329. [PubMed: 29854710]

97. Walter SS, Maurer M, Storz C, et al. Effects of Radiation Dose Reduction on Diagnostic Accuracy of Abdominal CT in Young Adults with Suspected Acute Diverticulitis: A Retrospective Intraindividual Analysis. Academic radiology. 2018.

98. Cirocchi R, Randolph JJ, Binda GA, et al. Is the outpatient management of acute diverticulitis safe and effective? A systematic review and meta-analysis. Tech Coloproctol. 2019;23(2):87-100. [PubMed: 30684110]

99. Biondo S, Golda T, Kreisler E, et al. Outpatient versus hospitalization management for uncomplicated diverticulitis: a prospective, multicenter randomized clinical trial (DIVER Trial). Ann Surg. 2014;259(1):38-44. [PubMed: 23732265] 
100. Balasubramanian I, Fleming C, Mohan HM, Schmidt K, Haglind E, Winter DC. Out-Patient Management of Mild or Uncomplicated Diverticulitis: A Systematic Review. Digestive surgery. 2017;34(2):151-160. [PubMed: 27701164]

101. Moya P, Arroyo A, Perez-Legaz J, et al. Applicability, safety and efficiency of outpatient treatment in uncomplicated diverticulitis. Tech Coloproctol. 2012;16(4):301-307. [PubMed: 22706731]

102. Rueda JC, Jimenez A, Caro A, et al. Home treatment of uncomplicated acute diverticulitis. International surgery. 2012;97(3):203-209. [PubMed: 23113847]

103. Jaung R, Robertson J, Rowbotham D, Bissett I. Current management of acute diverticulitis: a survey of Australasian surgeons. The New Zealand medical journal. 2016;129(1431):23-29.

104. Khan DZ, Kelly ME, O'Reilly J, et al. A national evaluation of the management practices of acute diverticulitis. The surgeon : journal of the Royal Colleges of Surgeons of Edinburgh and Ireland. 2017;15(4):206-210. [PubMed: 26791395]

105. Vennix S, Morton DG, Hahnloser D, Lange JF, Bemelman WA. Systematic review of evidence and consensus on diverticulitis: an analysis of national and international guidelines. Colorectal Dis. 2014;16(11):866-878. [PubMed: 24801825]

106. Alonso S, Pera M, Parés D, et al. Outpatient treatment of patients with uncomplicated acute diverticulitis. 2010;12(10Online):e278-e282.

107. Sanchez-Velazquez P, Grande L, Pera M. Outpatient treatment of uncomplicated diverticulitis: a systematic review. Eur J Gastroenterol Hepatol. 2016;28(6):622-627. [PubMed: 26891198]

108. Chabok A, Pahlman L, Hjern F, Haapaniemi S, Smedh K. Randomized clinical trial of antibiotics in acute uncomplicated diverticulitis. Br J Surg. 2012;99(4):532-539. [PubMed: 22290281]

109. Daniels L, Unlu C, de Korte N, et al. Randomized clinical trial of observational versus antibiotic treatment for a first episode of CT-proven uncomplicated acute diverticulitis. Br J Surg. 2017;104(1):52-61. [PubMed: 27686365]

110. Sharma PV, Eglinton T, Hider P, Frizelle F. Systematic review and meta-analysis of the role of routine colonic evaluation after radiologically confirmed acute diverticulitis. Ann Surg. 2014;259(2):263-272. [PubMed: 24169174]

111. Westwood DA, Eglinton TW, Frizelle FA. Routine colonoscopy following acute uncomplicated diverticulitis. Br J Surg. 2011;98(11):1630-1634. [PubMed: 21713756]

112. Abbas MA, Cannom RR, Chiu VY, et al. Triage of patients with acute diverticulitis: are some inpatients candidates for outpatient treatment? Colorectal Dis. 2013;15(4):451-457. [PubMed: 23061533]

113. Mizuki A, Nagata H, Tatemichi M, et al. The out-patient management of patients with acute mildto-moderate colonic diverticulitis. Aliment Pharmacol Ther. 2005;21(7):889-897. [PubMed: 15801924]

114. Brandl A, Kratzer T, Kafka-Ritsch R, et al. Diverticulitis in immunosuppressed patients: A fatal outcome requiring a new approach? Canadian journal of surgery Journal canadien de chirurgie. 2016;59(4):254-261. [PubMed: 27240131]

115. McIntyre LK, Arbabi S, Robinson EF, Maier RV. Analysis of Risk Factors for Patient Readmission 30 Days Following Discharge From General SurgeryRisk Factors for 30-Day Readmissions to General SurgeryRisk Factors for 30-Day Readmissions to General Surgery. JAMA Surgery. 2016;151(9):855-861. [PubMed: 27303913]

116. Tan JP, Barazanchi AW, Singh PP, Hill AG, Maccormick AD. Predictors of acute diverticulitis severity: A systematic review. International journal of surgery (London, England). 2016;26:4352.

117. Bolkenstein HE, van de Wall BJM, Consten ECJ, Broeders I, Draaisma WA. Risk factors for complicated diverticulitis: systematic review and meta-analysis. Int J Colorectal Dis. 2017;32(10):1375-1383. [PubMed: 28799055]

118. Jaung R, Kularatna M, Robertson JP, et al. Uncomplicated Acute Diverticulitis: Identifying Risk Factors for Severe Outcomes. World J Surg. 2017;41(9):2258-2265. [PubMed: 28401253]

119. Yoo T, Yang KH, Kim J, et al. Predictive Factors Affecting the Clinical Course of Patients With Diverticulitis: Who Needs Hospital Management? Annals of coloproctology. 2018;34(1):23-28. [PubMed: 29535984] 
120. Richards SJG, Frizelle FA, Geddes JA, Eglinton TW, Hampton MB. Frailty in surgical patients. Int J Colorectal Dis. 2018;33(12):1657-1666. [PubMed: 30218144]

121. Ward MAR, Alenazi A, Delisle M, Logsetty S. The impact of frailty on acute care general surgery patients: A systematic review. The journal of trauma and acute care surgery. 2019;86(1):148-154. [PubMed: 30399129]

122. Aune D, Sen A, Norat T, Riboli E. Dietary fibre intake and the risk of diverticular disease: a systematic review and meta-analysis of prospective studies. Eur J Nutr. 2020;59(2):421-432.

123. Painter NS, Burkitt DP. Diverticular disease of the colon, a 20th century problem. Clin Gastroenterol. 1975;4(1):3-21. [PubMed: 1109818]

124. Aldoori WH, Giovannucci EL, Rimm EB, Wing AL, Trichopoulos DV, Willett WC. A prospective study of alcohol, smoking, caffeine, and the risk of symptomatic diverticular disease in men. Ann Epidemiol. 1995;5(3):221-228. [PubMed: 7606311]

125. Turunen P, Wikstrom H, Carpelan-Holmstrom M, Kairaluoma P, Kruuna O, Scheinin T. Smoking increases the incidence of complicated diverticular disease of the sigmoid colon. Scand J Surg. 2010;99(1):14-17. [PubMed: 20501352]

126. Aune D, Sen A, Leitzmann MF, Norat T, Tonstad S, Vatten LJ. Body mass index and physical activity and the risk of diverticular disease: a systematic review and meta-analysis of prospective studies. Eur J Nutr. 2017;56(8):2423-2438. [PubMed: 28393286]

127. Biondo S, Lopez Borao J, Millan M, Kreisler E, Jaurrieta E. Current status of the treatment of acute colonic diverticulitis: a systematic review. Colorectal Dis. 2012;14(1):e1-e11. [PubMed: 21848896]

128. Rafferty J, Shellito P, Hyman NH, Buie WD. Practice parameters for sigmoid diverticulitis. Dis Colon Rectum. 2006;49(7):939-944. [PubMed: 16741596]

129. Humes DJ, Simpson J, Smith J, et al. Visceral hypersensitivity in symptomatic diverticular disease and the role of neuropeptides and low grade inflammation. Neurogastroenterology and motility : the official journal of the European Gastrointestinal Motility Society. 2012;24(4):318e163. [PubMed: 22276853]

130. van Dijk ST, Daniels L, de Korte N, et al. Quality of Life and Persistent Symptoms After Uncomplicated Acute Diverticulitis. Dis Colon Rectum. 2019;62(5):608-614. [PubMed: 30807455]

131. Trepsi E, Colla C, Panizza P, et al. [Therapeutic and prophylactic role of mesalazine (5-ASA) in symptomatic diverticular disease of the large intestine. 4 year follow-up results]. Minerva Gastroenterol Dietol. 1999;45(4):245-252. [PubMed: 16498335]

132. Tursi A, Brandimarte G, Daffina R. Long-term treatment with mesalazine and rifaximin versus rifaximin alone for patients with recurrent attacks of acute diverticulitis of colon. Digestive and liver disease : official journal of the Italian Society of Gastroenterology and the Italian Association for the Study of the Liver. 2002;34(7):510-515.

133. Kruis W, Kardalinos V, Eisenbach T, et al. Randomised clinical trial: mesalazine versus placebo in the prevention of diverticulitis recurrence. Aliment Pharmacol Ther. 2017;46(3):282-291. [PubMed: 28543263]

134. Raskin JB, Kamm MA, Jamal MM, et al. Mesalamine did not prevent recurrent diverticulitis in phase 3 controlled trials. Gastroenterology. 2014;147(4):793-802. [PubMed: 25038431]

135. Urushidani S, Kuriyama A, Matsumura M. 5-aminosalicylic acid agents for prevention of recurrent diverticulitis: A systematic review and meta-analysis. J Gastroenterol Hepatol. 2018;33(1):12-19.

136. Bianchi M, Festa V, Moretti A, et al. Meta-analysis: long-term therapy with rifaximin in the management of uncomplicated diverticular disease. Aliment Pharmacol Ther. 2011;33(8):902910. [PubMed: 21366632]

137. Lahner E, Bellisario C, Hassan C, Zullo A, Esposito G, Annibale B. Probiotics in the Treatment of Diverticular Disease. A Systematic Review. Journal of gastrointestinal and liver diseases : JGLD. 2016;25(1):79-86. [PubMed: 27014757]

138. Ojetti V, Petruzziello C, Cardone S, et al. The Use of Probiotics in Different Phases of Diverticular Disease. Reviews on recent clinical trials. 2018;13(2):89-96. [PubMed: 29607785]

Curr Probl Surg. Author manuscript; available in PMC 2021 October 01. 
139. Biondo S, Pares D, Marti Rague J, Kreisler E, Fraccalvieri D, Jaurrieta E. Acute colonic diverticulitis in patients under 50 years of age. Br J Surg. 2002;89(9):1137-1141. [PubMed: 12190679]

140. Eusebio EB, Eisenberg MM. Natural history of diverticular disease of the colon in young patients. Am J Surg. 1973;125(3):308-311. [PubMed: 4690116]

141. Makela J, Vuolio S, Kiviniemi H, Laitinen S. Natural history of diverticular disease: when to operate? Dis Colon Rectum. 1998;41(12):1523-1528. [PubMed: 9860333]

142. Practice parameters for the treatment of sigmoid diverticulitis. The Standards Task Force. The American Society of Colon and Rectal Surgeons. Dis Colon Rectum. 2000;43(3):289. [PubMed: 10733107]

143. Devaraj B, Liu W, Tatum J, Cologne K, Kaiser AM. Medically Treated Diverticular Abscess Associated With High Risk of Recurrence and Disease Complications. Dis Colon Rectum. 2016;59(3):208-215. [PubMed: 26855395]

144. van de Wall BJM, Stam MAW, Draaisma WA, et al. Surgery versus conservative management for recurrent and ongoing left-sided diverticulitis (DIRECT trial): an open-label, multicentre, randomised controlled trial. Lancet Gastroenterol Hepatol. 2017;2(1):13-22. [PubMed: 28404008]

145. Chodak GW, Rangel DM, Passaro E Jr. Colonic diverticulitis in patients under age 40: need for earlier diagnosis. Am J Surg. 1981;141(6):699-702. [PubMed: 7246860]

146. Freischlag J, Bennion RS, Thompson JE, Jr. Complications of diverticular disease of the colon in young people. Dis Colon Rectum. 1986;29(10):639-643. [PubMed: 3757703]

147. Nelson RS, Velasco A, Mukesh BN. Management of diverticulitis in younger patients. Dis Colon Rectum. 2006;49(9):1341-1345. [PubMed: 16897326]

148. Hall JF. Expert Commentary on Uncomplicated Sigmoid Diverticulitis. Dis Colon Rectum. 2018;61(10):1144-1145. [PubMed: 30192322]

149. Klarenbeek BR, Veenhof AA, Bergamaschi R, et al. Laparoscopic sigmoid resection for diverticulitis decreases major morbidity rates: a randomized control trial: short-term results of the Sigma Trial. Ann Surg. 2009;249(1):39-44. [PubMed: 19106674]

150. Klarenbeek BR, Bergamaschi R, Veenhof AA, et al. Laparoscopic versus open sigmoid resection for diverticular disease: follow-up assessment of the randomized control Sigma trial. Surg Endosc. 2011;25(4):1121-1126. [PubMed: 20872022]

151. Benn PL, Wolff BG, Ilstrup DM. Level of anastomosis and recurrent colonic diverticulitis. Am J Surg. 1986;151(2):269-271. [PubMed: 3946763]

152. Thaler K, Baig MK, Berho M, et al. Determinants of recurrence after sigmoid resection for uncomplicated diverticulitis. Dis Colon Rectum. 2003;46(3):385-388. [PubMed: 12626916]

153. Lehmann RK, Brounts LR, Johnson EK, Rizzo JA, Steele SR. Does sacrifice of the inferior mesenteric artery or superior rectal artery affect anastomotic leak following sigmoidectomy for diverticulitis? a retrospective review. Am J Surg. 2011;201(5):623-627. [PubMed: 21545911]

154. Cirocchi R, Trastulli S, Farinella E, et al. Is inferior mesenteric artery ligation during sigmoid colectomy for diverticular disease associated with increased anastomotic leakage? A metaanalysis of randomized and non-randomized clinical trials. Colorectal Dis. 2012;14(9):e521-529. [PubMed: 22632654]

155. Masoni L, Mari FS, Nigri G, et al. Preservation of the inferior mesenteric artery via laparoscopic sigmoid colectomy performed for diverticular disease: real benefit or technical challenge: a randomized controlled clinical trial. Surg Endosc. 2013;27(1):199-206. [PubMed: 22733197]

156. Araujo SE, Seid VE, Kim NJ, Bertoncini AB, Nahas SC, Cecconello I. Assessing the extent of colon lengthening due to splenic flexure mobilization techniques: a cadaver study. Arquivos de gastroenterologia. 2012;49(3):219-222. [PubMed: 23011246]

157. Gouvas N, Gogos-Pappas G, Tsimogiannis K, et al. Impact of splenic flexure mobilization on short-term outcomes after laparoscopic left colectomy for colorectal cancer. Surg Laparosc Endosc Percutan Tech. 2014;24(5):470-474. [PubMed: 24710257]

158. Marsden MR, Conti JA, Zeidan S, et al. The selective use of splenic flexure mobilization is safe in both laparoscopic and open anterior resections. Colorectal Dis. 2012;14(10):1255-1261. [PubMed: 22188371] 
159. Schlussel AT, Wiseman JT, Kelly JF, et al. Location is everything: The role of splenic flexure mobilization during colon resection for diverticulitis. International journal of surgery (London, England). 2017;40:124-129.

160. Ricciardi R, Roberts PL, Marcello PW, Hall JF, Read TE, Schoetz DJ. Anastomotic leak testing after colorectal resection: what are the data? Arch Surg. 2009;144(5):407-411; discussion 411402. [PubMed: 19451481]

161. Klarenbeek BR, de Korte N, van der Peet DL, Cuesta MA. Review of current classifications for diverticular disease and a translation into clinical practice. International journal of colorectal disease. 2012;27(2):207-214. [PubMed: 21928041]

162. Roberts P Changing Paradigms in the Treatment of Sigmoid Diverticulitis. London: Spinger; 2010.

163. Bahadursingh AM, Longo WE. Colovaginal fistulas. Etiology and management. The Journal of reproductive medicine. 2003;48(7):489-495. [PubMed: 12953321]

164. Melchior S, Cudovic D, Jones J, Thomas C, Gillitzer R, Thüroff J. Diagnosis and surgical management of colovesical fistulas due to sigmoid diverticulitis. The Journal of urology. 2009;182(3):978-982. [PubMed: 19616793]

165. Pontari MA, McMillen MA, Garvey RH, Ballantyne GH. Diagnosis and treatment of enterovesical fistulae. The American surgeon. 1992;58(4):258-263. [PubMed: 1586086]

166. Woods RJ, Lavery IC, Fazio VW, Jagelman DG, Weakley FL. Internal fistulas in diverticular disease. Diseases of the colon and rectum. 1988;31(8):591-596. [PubMed: 3402284]

167. Garcea G, Majid I, Sutton CD, Pattenden CJ, Thomas WM. Diagnosis and management of colovesical fistulae; six-year experience of 90 consecutive cases. Colorectal disease : the official journal of the Association of Coloproctology of Great Britain and Ireland. 2006;8(4):347-352. [PubMed: 16630242]

168. Smeenk RM, Plaisier PW, van der Hoeven JAB, Hesp WLEM. Outcome of surgery for colovesical and colovaginal fistulas of diverticular origin in 40 patients. Journal of gastrointestinal surgery : official journal of the Society for Surgery of the Alimentary Tract. 2012;16(8):1559-1565. [PubMed: 22653331]

169. Hjern F, Goldberg SM, Johansson C, Parker SC, Mellgren A. Management of diverticular fistulae to the female genital tract. Colorectal disease : the official journal of the Association of Coloproctology of Great Britain and Ireland. 2007;9(5):438-442. [PubMed: 17504341]

170. Berger MB, Khandwala N, Fenner DE, Burney RE. Colovaginal Fistulas: Presentation, Evaluation, and Management. Female pelvic medicine \& reconstructive surgery. 2016;22(5):355358. [PubMed: 27171321]

171. Fazio VW, Church JM, Jagelman DG, et al. Colocutaneous fistulas complicating diverticulitis. Diseases of the colon and rectum. 1987;30(2):89-94. [PubMed: 3803127]

172. Vasilevsky CA, Belliveau P, Trudel JL, Stein BL, Gordon PH. Fistulas complicating diverticulitis. International journal of colorectal disease. 1998;13(2):57-60. [PubMed: 9638488]

173. Ahmad DS, Quist EE, Hutchins GF, Bhat I. Coloenteric fistula in a young patient with recurrent diverticulitis: A case report and review of the literature. The Netherlands journal of medicine. 2016;74(8):358-261. [PubMed: 27762218]

174. Hool GJ, Bokey EL, Pheils MT. Diverticular colo-enteric fistulae. The Australian and New Zealand journal of surgery. 1981;51(4):358-359. [PubMed: 6944054]

175. Jensen DM, Ohning GV, Kovacs TOG, et al. Natural history of definitive diverticular hemorrhage based on stigmata of recent hemorrhage and colonoscopic Doppler blood flow monitoring for risk stratification and definitive hemostasis. Gastrointestinal endoscopy. 2016;83(2):416-423. [PubMed: 26227931]

176. Yuhara H, Corley DA, Nakahara F, et al. Aspirin and non-aspirin NSAIDs increase risk of colonic diverticular bleeding: a systematic review and meta-analysis. Journal of gastroenterology. 2014;49(6):992-1000. [PubMed: 24221694]

177. Jensen DM. Diagnosis and treatment of definitive diverticular hemorrhage (DDH). The American journal of gastroenterology. 2018;113(11):1570-1573. [PubMed: 29748559]

178. Fujino Y, Inoue Y, Onodera M, et al. Risk factors for early re-bleeding and associated hospitalization in patients with colonic diverticular bleeding. Colorectal disease : the official 
journal of the Association of Coloproctology of Great Britain and Ireland. 2013;15(8):982-986. [PubMed: 23560619]

179. Niikura R, Nagata N, Yamada A, Akiyama J, Shimbo T, Uemura N. Recurrence of colonic diverticular bleeding and associated risk factors. Colorectal disease : the official journal of the Association of Coloproctology of Great Britain and Ireland. 2012;14(3):302-305. [PubMed: 21692963]

180. Strate LL, Gralnek IM. ACG Clinical Guideline: Management of Patients With Acute Lower Gastrointestinal Bleeding. The American journal of gastroenterology. 2016;111(5):755. [PubMed: 27151132]

181. Sugrue J, Lee J, Warner C, et al. Acute diverticulitis in renal transplant patients: should we treat them differently? Surgery. 2018;163(4):857-865. [PubMed: 29289391]

182. Jang HJ, Lim HK, Lee SJ, Lee WJ, Kim EY, Kim SH. Acute diverticulitis of the cecum and ascending colon: the value of thin-section helical CT findings in excluding colonic carcinoma. AJR American journal of roentgenology. 2000;174(5):1397-1402. [PubMed: 10789802]

183. Agarwal AK, Karanjawala BE, Maykel JA, Johnson EK, Steele SR. Routine colonic endoscopic evaluation following resolution of acute diverticulitis: is it necessary? World journal of gastroenterology. 2014;20(35):12509-12516. [PubMed: 25253951]

184. Levin TR, Zhao W, Conell C, et al. Complications of colonoscopy in an integrated health care delivery system. Annals of internal medicine. 2006;145(12):880-886. [PubMed: 17179057]

185. Sai VF, Velayos F, Neuhaus J, Westphalen AC. Colonoscopy after CT diagnosis of diverticulitis to exclude colon cancer: a systematic literature review. Radiology. 2012;263(2):383-390. [PubMed: 22517956]

186. Lahat A, Yanai H, Menachem Y, Avidan B, Bar-Meir S. The feasibility and risk of early colonoscopy in acute diverticulitis: a prospective controlled study. Endoscopy. 2007;39(6):521524. [PubMed: 17554647]

187. Lam TJ, Meurs-Szojda MM, Gundlach L, et al. There is no increased risk for colorectal cancer and adenomas in patients with diverticulitis: a retrospective longitudinal study. Colorectal disease : the official journal of the Association of Coloproctology of Great Britain and Ireland. 2010;12(11):1122-1126. [PubMed: 19575738]

188. Lau KC, Spilsbury K, Farooque Y, et al. Is colonoscopy still mandatory after a CT diagnosis of left-sided diverticulitis: can colorectal cancer be confidently excluded? Diseases of the colon and rectum. 2011;54(10):1265-1270. [PubMed: 21904141]

189. Schmilovitz-Weiss H, Yalunin E, Boaz M, et al. Does a colonoscopy after acute diverticulitis affect its management?: a single center experience. Journal of clinical gastroenterology. 2012;46(4):317-320. [PubMed: 22186742]

190. Zaman S, Chapman W, Mohammed I, Gill K, Ward ST. Patients with computed tomographyproven acute diverticulitis require follow-up to exclude colorectal cancer. Intest Res. 2017;15(2):195-202. [PubMed: 28522949]

191. Rottier ST, van Dijk AAW, van Geloven WH, et al. S. J. M. Boermeester. Meta- analysis of the role of colonoscopy after an episode of left- sided acute diverticulitis. Vol 1062019.

192. de Vries HS, Boerma D, Timmer R, van Ramshorst B, Dieleman LA, van Westreenen HL. Routine colonoscopy is not required in uncomplicated diverticulitis: a systematic review. Surgical endoscopy. 2014;28(7):2039-2047. [PubMed: 24488358]

193. Asaad P, Hajibandeh S, Rahm M, Johnston T, Chowdhury S, Bronder C. Should a colonoscopy be offered routinely to patients with CT proven acute diverticulitis? A retrospective cohort study and meta-analysis of best available evidence. World J Gastrointest Endosc. 2019;11(7):427-437. [PubMed: 31367268]

194. Lecleire S, Nahon S, Alatawi A, et al. Diagnostic impact of routine colonoscopy following acute diverticulitis: A multicenter study in 808 patients and controls. United European gastroenterology journal. 2014;2(4):301-306. [PubMed: 25083288]

195. Chan DKH, Tan KK. There Is No Role for Colonoscopy after Diverticulitis among Asian Patients Less than 50 Years of Age. Gastrointest Tumors. 2017;3(3-4):136-140. [PubMed: 28611980] 
196. Francis NK, Sylla P, Abou-Khalil M, et al. EAES and SAGES 2018 consensus conference on acute diverticulitis management: evidence-based recommendations for clinical practice. Surgical endoscopy. 2019;33(9):2726-2741. [PubMed: 31250244]

197. Biondo S, Trenti L, Elvira J, Golda T, Kreisler E. Outcomes of colonic diverticulitis according to the reason of immunosuppression. Am J Surg. 2016;212(3):384-390. [PubMed: 27255782]

198. Perkins JD, Shield CF, 3rd, Chang FC, Farha GJ. Acute diverticulitis. Comparison of treatment in immunocompromised and nonimmunocompromised patients. Am J Surg. 1984;148(6):745-748. [PubMed: 6507744]

199. Regenbogen SE, Hardiman KM, Hendren S, Morris AM. Surgery for diverticulitis in the 21 st century: a systematic review. JAMA Surg. 2014;149(3):292-303. [PubMed: 24430164]

200. Slieker JC, Komen N, Mannaerts GH, et al. Long-term and perioperative corticosteroids in anastomotic leakage: a prospective study of 259 left-sided colorectal anastomoses. Arch Surg. 2012;147(5):447-452. [PubMed: 22249852]

201. Madiba TE, Muckart DJ, Thomson SR. Human immunodeficiency disease: how should it affect surgical decision making? World J Surg. 2009;33(5):899-909. [PubMed: 19280251]

202. Collaboration NCDRF. Trends in adult body-mass index in 200 countries from 1975 to 2014: a pooled analysis of 1698 population-based measurement studies with 19.2 million participants. Lancet. 2016;387(10026):1377-1396. [PubMed: 27115820]

203. Alizadeh RF, Moghadamyeghaneh Z, Whealon MD, et al. Body Mass Index Significantly Impacts Outcomes of Colorectal Surgery. Am Surg. 2016;82(10):930-935. [PubMed: 27779976]

204. Makary MA, Segev DL, Pronovost PJ, et al. Frailty as a predictor of surgical outcomes in older patients. J Am Coll Surg. 2010;210(6):901-908. [PubMed: 20510798]

205. Robinson TN, Walston JD, Brummel NE, et al. Frailty for Surgeons: Review of a National Institute on Aging Conference on Frailty for Specialists. J Am Coll Surg. 2015;221(6):10831092. [PubMed: 26422746]

206. Fried LP, Tangen CM, Walston J, et al. Frailty in older adults: evidence for a phenotype. J Gerontol A Biol Sci Med Sci. 2001;56(3):M146-156. [PubMed: 11253156]

207. Robinson TN, Wu DS, Pointer L, Dunn CL, Cleveland JC, Jr., Moss M. Simple frailty score predicts postoperative complications across surgical specialties. Am J Surg. 2013;206(4):544550. [PubMed: 23880071]

208. Moriello C, Mayo NE, Feldman L, Carli F. Validating the six-minute walk test as a measure of recovery after elective colon resection surgery. Arch Phys Med Rehabil. 2008;89(6):1083-1089. [PubMed: 18503803]

209. Bolshinsky V, Li MH, Ismail H, Burbury K, Riedel B, Heriot A. Multimodal Prehabilitation Programs as a Bundle of Care in Gastrointestinal Cancer Surgery: A Systematic Review. Dis Colon Rectum. 2018;61(1):124-138. [PubMed: 29219922]

210. White JV, Guenter P, Jensen G, et al. Consensus statement: Academy of Nutrition and Dietetics and American Society for Parenteral and Enteral Nutrition: characteristics recommended for the identification and documentation of adult malnutrition (undernutrition). JPEN J Parenter Enteral Nutr. 2012;36(3):275-283. [PubMed: 22535923]

211. van Stijn MF, Korkic-Halilovic I, Bakker MS, van der Ploeg T, van Leeuwen PA, Houdijk AP. Preoperative nutrition status and postoperative outcome in elderly general surgery patients: a systematic review. JPEN J Parenter Enteral Nutr. 2013;37(1):37-43. [PubMed: 22549764]

212. Braga M, Gianotti L, Nespoli L, Radaelli G, Di Carlo V. Nutritional approach in malnourished surgical patients: a prospective randomized study. Arch Surg. 2002;137(2):174-180. [PubMed: 11822956]

213. Baucom RB, Poulose BK, Herline AJ, Muldoon RL, Cone MM, Geiger TM. Smoking as dominant risk factor for anastomotic leak after left colon resection. Am J Surg. 2015;210(1):1-5. [PubMed: 25910885]

214. Sorensen LT. Wound healing and infection in surgery: the pathophysiological impact of smoking, smoking cessation, and nicotine replacement therapy: a systematic review. Ann Surg. 2012;255(6):1069-1079. [PubMed: 22566015] 
215. Myers K, Hajek P, Hinds C, McRobbie H. Stopping smoking shortly before surgery and postoperative complications: a systematic review and meta-analysis. Arch Intern Med. 2011;171(11):983-989. [PubMed: 21403009]

216. Ata A, Lee J, Bestle SL, Desemone J, Stain SC. Postoperative hyperglycemia and surgical site infection in general surgery patients. Arch Surg. 2010;145(9):858-864. [PubMed: 20855756]

217. Kwon S, Thompson R, Dellinger P, Yanez D, Farrohki E, Flum D. Importance of perioperative glycemic control in general surgery: a report from the Surgical Care and Outcomes Assessment Program. Ann Surg. 2013;257(1):8-14. [PubMed: 23235393]

218. Patel AY, Eagle KA, Vaishnava P. Cardiac risk of noncardiac surgery. J Am Coll Cardiol. 2015;66(19):2140-2148. [PubMed: 26541926]

219. Malinchoc M, Kamath PS, Gordon FD, Peine CJ, Rank J, ter Borg PC. A model to predict poor survival in patients undergoing transjugular intrahepatic portosystemic shunts. Hepatology. 2000;31(4):864-871. [PubMed: 10733541]

220. Pugh RN, Murray-Lyon IM, Dawson JL, Pietroni MC, Williams R. Transection of the oesophagus for bleeding oesophageal varices. Br J Surg. 1973;60(8):646-649. [PubMed: 4541913]

221. Meunier K, Mucci S, Quentin V, Azoulay R, Arnaud JP, Hamy A. Colorectal surgery in cirrhotic patients: assessment of operative morbidity and mortality. Dis Colon Rectum. 2008;51(8):12251231. [PubMed: 18521677]

222. Ghaferi AA, Mathur AK, Sonnenday CJ, Dimick JB. Adverse outcomes in patients with chronic liver disease undergoing colorectal surgery. Ann Surg. 2010;252(2):345-350. [PubMed: 20622652]

223. Abbas N, Makker J, Abbas H, Balar B. Perioperative Care of Patients With Liver Cirrhosis: A Review. Health Serv Insights. 2017;10:1178632917691270. [PubMed: 28469455]

224. Yang CK, Teng A, Lee DY, Rose K. Pulmonary complications after major abdominal surgery: National Surgical Quality Improvement Program analysis. J Surg Res. 2015;198(2):441-449. [PubMed: 25930169]

225. Hawkins AT, Geiger TM, King AB, et al. An enhanced recovery program in colorectal surgery is associated with decreased organ level rates of complications: a difference-in-differences analysis. Surg Endosc. 2019;33(7):2222-2230. [PubMed: 30334161]

226. Hewett PJ, Allardyce RA, Bagshaw PF, et al. Short-term outcomes of the Australasian randomized clinical study comparing laparoscopic and conventional open surgical treatments for colon cancer: the ALCCaS trial. Ann Surg. 2008;248(5):728-738. [PubMed: 18948799]

227. Veldkamp R, Kuhry E, Hop WC, et al. Laparoscopic surgery versus open surgery for colon cancer: short-term outcomes of a randomised trial. Lancet Oncol. 2005;6(7):477484.

228. Braga M, Frasson M, Zuliani W, Vignali A, Pecorelli N, Di Carlo V. Randomized clinical trial of laparoscopic versus open left colonic resection. Br J Surg. 2010;97(8):1180-1186. [PubMed: 20602506]

229. Lacy AM, Garcia-Valdecasas JC, Delgado S, et al. Laparoscopy-assisted colectomy versus open colectomy for treatment of non-metastatic colon cancer: a randomised trial. Lancet. 2002;359(9325):2224-2229. [PubMed: 12103285]

230. Yamamoto S, Inomata M, Katayama H, et al. Short-term surgical outcomes from a randomized controlled trial to evaluate laparoscopic and open D3 dissection for stage II/III colon cancer: Japan Clinical Oncology Group Study JCOG 0404. Ann Surg. 2014;260(1):23-30. [PubMed: 24509190]

231. Thiele RH, Rea KM, Turrentine FE, et al. Standardization of care: impact of an enhanced recovery protocol on length of stay, complications, and direct costs after colorectal surgery. $\mathrm{J}$ Am Coll Surg. 2015;220(4):430-443. [PubMed: 25797725]

232. Bakker N, Cakir H, Doodeman HJ, Houdijk AP. Eight years of experience with Enhanced Recovery After Surgery in patients with colon cancer: Impact of measures to improve adherence. Surgery. 2015;157(6):1130-1136. [PubMed: 25791027]

233. Larson DW, Lovely JK, Cima RR, et al. Outcomes after implementation of a multimodal standard care pathway for laparoscopic colorectal surgery. Br J Surg. 2014;101(8):1023-1030. [PubMed: 24828373] 
234. Boelens PG, Heesakkers FF, Luyer MD, et al. Reduction of postoperative ileus by early enteral nutrition in patients undergoing major rectal surgery: prospective, randomized, controlled trial. Ann Surg. 2014;259(4):649-655. [PubMed: 24169163]

235. Castelino T, Fiore JF, Jr., Niculiseanu P, Landry T, Augustin B, Feldman LS. The effect of early mobilization protocols on postoperative outcomes following abdominal and thoracic surgery: A systematic review. Surgery. 2016;159(4):991-1003. [PubMed: 26804821]

236. Kim JH, Cheon JH, Park S, et al. Relationship between disease location and age, obesity, and complications in Korean patients with acute diverticulitis: a comparison of clinical patterns with those of Western populations. Hepatogastroenterology. 2008;55(84):983-986. [PubMed: 18705312]

237. Markham NI, Li AK. Diverticulitis of the right colon--experience from Hong Kong. Gut. 1992;33(4):547-549. [PubMed: 1582600]

238. Lin OS, Soon MS, Wu SS, Chen YY, Hwang KL, Triadafilopoulos G. Dietary habits and rightsided colonic diverticulosis. Dis Colon Rectum. 2000;43(10):1412-1418. [PubMed: 11052519]

239. Puylaert JB. Ultrasound of colon diverticulitis. Digestive diseases (Basel, Switzerland). 2012;30(1):56-59.

240. Jensen DM, Machicado GA, Jutabha R, Kovacs TO. Urgent colonoscopy for the diagnosis and treatment of severe diverticular hemorrhage. N Engl J Med. 2000;342(2):78-82. [PubMed: 10631275]

241. JA W, CS A, TMAJ vV, TSDV R, HJV Z-L, H vG. Disease Course of Right- and Left-sided Diverticulitis in a Western Population. 2016;6(2):421.

242. Shyung LR, Lin SC, Shih SC, Kao CR, Chou SY. Decision making in right-sided diverticulitis. World J Gastroenterol. 2003;9(3):606-608. [PubMed: 12632528]

243. Stollman N, Smalley W, Hirano I, Committee AICG. American Gastroenterological Association Institute Guideline on the Management of Acute Diverticulitis. Gastroenterology. 2015;149(7):1944-1949. [PubMed: 26453777]

244. Cobben LP, Groot I, Blickman JG, Puylaert JB. Right colonic diverticulitis: MR appearance. Abdom Imaging. 2003;28(6):794-798. [PubMed: 14753592]

245. Luoma A, Nagy AG. Cecal diverticulitis. Can J Surg. 1989;32(4):283-286. [PubMed: 2736455]

246. Jang HJ, Lim HK, Lee SJ, Lee WJ, Kim EY, Kim SH. Acute diverticulitis of the cecum and ascending colon: the value of thin-section helical CT findings in excluding colonic carcinoma. AJR Am J Roentgenol. 2000;174(5):1397-1402. [PubMed: 10789802]

247. Lee JH, Park YS, Choi JS. The epidemiology of appendicitis and appendectomy in South Korea: national registry data. J Epidemiol. 2010;20(2):97-105. [PubMed: 20023368]

248. Phillips BJ, Perry CW. Appendiceal diverticulitis. Mayo Clin Proc. 1999;74(9):890-892. [PubMed: 10488790]

249. Cristaudo A, Pillay P, Naidu S. Caecal diverticulitis: Presentation and management. Ann Med Surg (Lond). 2015;4(1):72-75. [PubMed: 25830021]

250. Sugihara K, Muto T, Morioka Y, Asano A, Yamamoto T. Diverticular disease of the colon in Japan. A review of 615 cases. Dis Colon Rectum. 1984;27(8):531-537. [PubMed: 6468190]

251. Faucheron JL, Roblin X, Bichard P, Heluwaert F. The prevalence of right-sided colonic diverticulosis and diverticular haemorrhage. Colorectal Dis. 2013;15(5):e266-270. [PubMed: 23350931]

252. Rao PM, Rhea JT, Novelline RA, Mostafavi AA, McCabe CJ. Effect of computed tomography of the appendix on treatment of patients and use of hospital resources. N Engl J Med. 1998;338(3):141-146. [PubMed: 9428814]

253. Chou YH, Chiou HJ, Tiu CM, et al. Sonography of acute right side colonic diverticulitis. Am J Surg. 2001;181(2):122-127. [PubMed: 11425051]

254. Komuta K, Yamanaka S, Okada K, et al. Toward therapeutic guidelines for patients with acute right colonic diverticulitis. Am J Surg. 2004;187(2):233-237. [PubMed: 14769311]

255. Oudenhoven LF, Koumans RK, Puylaert JB. Right colonic diverticulitis: US and CT findings-new insights about frequency and natural history. Radiology. 1998;208(3):611-618. [PubMed: 9722836] 
256. Park SJ, Choi SI, Lee SH, Lee KY. Image-guided conservative management of right colonic diverticulitis. World J Gastroenterol. 2009;15(46):5838-5842. [PubMed: 19998506]

257. Harada RN, Whelan TJ. Surgical management of cecal diverticulitis. Am J Surg. 1993;166(6):666-669; discussion 669-671. [PubMed: 8273846]

258. Tan KK, Wong J, Yan Z, Chong CS, Liu JZ, Sim R. Colonic diverticulitis in young Asians: a predominantly mild and right-sided disease. ANZ J Surg. 2014;84(3):181-184. [PubMed: 23796226]

259. Ngoi SS, Chia J, Goh MY, Sim E, Rauff A. Surgical management of right colon diverticulitis. Dis Colon Rectum. 1992;35(8):799-802. [PubMed: 1644006]

260. Poon RT, Chu KW. Inflammatory cecal masses in patients presenting with appendicitis. World J Surg. 1999;23(7):713-716; discussion 716. [PubMed: 10390592]

261. Kwon JW, Kim BS, Park HC, et al. Surgical treatment of complicated right colonic diverticulitis: laparoscopic versus open surgery. Surg Endosc. 2012;26(10):2926-2930. [PubMed: 22538691]

262. Zeina AR, Nachtigal A, Matter I, et al. Giant colon diverticulum: clinical and imaging findings in 17 patients with emphasis on CT criteria. Clin Imaging. 2013;37(4):704-710. [PubMed: 23312457]

263. Nigri G, Petrucciani N, Giannini G, et al. Giant colonic diverticulum: clinical presentation, diagnosis and treatment: systematic review of 166 cases. World J Gastroenterol. 2015;21(1):360 368. [PubMed: 25574112]

264. Steenvoorde P, Vogelaar FJ, Oskam J, Tollenaar RA. Giant colonic diverticula. Review of diagnostic and therapeutic options. Dig Surg. 2004;21(1):1-6; discussion 6. [PubMed: 14631129]

265. Macht R, Sheldon HK, Fisichella PM. Giant Colonic Diverticulum: a Rare Diagnostic and Therapeutic Challenge of Diverticular Disease. J Gastrointest Surg. 2015;19(8):1559-1560. [PubMed: 25681218]

266. Naber A, Sliutz AM, Freitas H. Giant diverticulum of the sigmoid colon. Br J Surg. 1995;82(7):985. [PubMed: 7648126]

267. de Oliveira NC, Welch JP. Giant diverticula of the colon: a clinical assessment. Am J Gastroenterol. 1997;92(7):1092-1096. [PubMed: 9219776]

268. MMP B, G B. Diverticules giants due sigmoide. Arch Mal Appar Dig Mal Nutr. 1946;35(353$355)$.

269. HUGHES WL, GREENE RC. Solitary air cyst of peritoneal cavity. AMA Arch Surg. 1953;67(6):931-936. [PubMed: 13103964]

270. Choong CK, Frizelle FA. Giant colonic diverticulum: report of four cases and review of the literature. Dis Colon Rectum. 1998;41(9):1178-1185; discussion 1185-1176. [PubMed: 9749503]

271. McNutt R, Schmitt D, Schulte W. Giant colonic diverticula--three distinct entities. Report of a case. Dis Colon Rectum. 1988;31(8):624-628. [PubMed: 3402287]

272. Mulder JW, Offerhaus GJ, Drillenburg P, Busch OR. "Giant diverticulum” sigmoid colon. J Am Coll Surg. 2002;195(1):130. [PubMed: 12113537]

273. Rosen NG, Gibbs DL, Soffer SZ, Valderrama E, Lee TK. Uroepithelium in a colonic diverticulum. J Pediatr Surg. 2000;35(9):1375-1376. [PubMed: 10999705]

274. Thomas S, Peel RL, Evans LE, Haarer KA. Best cases from the AFIP: Giant colonic diverticulum. Radiographics. 2006;26(6):1869-1872. [PubMed: 17102056]

275. Mainzer F, Minagi H. Giant sigmoid diverticulum. Am J Roentgenol Radium Ther Nucl Med. 1971;113(2):352-354.

276. Kricun R, Stasik JJ, Reither RD, Dex WJ. Giant colonic diverticulum. AJR Am J Roentgenol. 1980;135(3):507-512. [PubMed: 6773370]

277. Arima N, Tanimoto A, Hamada T, Sasaguri Y, Sasaki E, Shimokobe T. MALT lymphoma arising in giant diverticulum of ascending colon. Am J Gastroenterol. 2000;95(12):3673-3674. [PubMed: 11151934]

278. Chaiyasate K, Yavuzer R, Mittal V. Giant sigmoid diverticulum. Surgery. 2006;139(2):276-277. [PubMed: 16455339] 
279. Majeski J, Durst G. Obstructing giant colonic diverticulum. South Med J. 2000;93(8):797-799. [PubMed: 10963512]

280. Toiber-Levy M, Golffier-Rosete C, Martínez-Munive A, et al. Giant sigmoid diverticulum: case report and review of the literature. Gastroenterol Clin Biol. 2008;32(6-7):581-584. [PubMed: 18353583]

281. Kuganeswaran E, Fisher JK. Giant sigmoid diverticulum: a rare manifestation of diverticular disease. South Med J. 1998;91(10):952-955. [PubMed: 9786292]

282. Custer TJ, Blevins DV, Vara TM. Giant colonic diverticulum: a rare manifestation of a common disease. J Gastrointest Surg. 1999;3(5):543-548. [PubMed: 10482713]

283. Jasper DR, Weinstock LB, Balfe DM, Heiken J, Lyss CA, Silvermintz SD. Transverse colon diverticulitis: successful nonoperative management in four patients. Report of four cases. Dis Colon Rectum. 1999;42(7):955-958. [PubMed: 10411445]

284. Wilkinson TR, Wilkinson AR. Perforated diverticulum of the transverse colon. Saudi J Gastroenterol. 2007;13(4):194-196. [PubMed: 19858646]

285. GF T, PF F. Perforated solitary diverticulum of the transverse colon. The American Journal of Surgery. 1944;66(2):280-283.

286. Rao UP, Venkitachalam PS, Posner GL, Estuita ET. Diverticulitis manifesting as transverse colocutaneous fistula: report of a case and review of literature. Dis Colon Rectum. 1980;23(1):44-48. [PubMed: 7379651]

287. Yamamoto M, Okamura T, Tomikawa M, et al. Perforated diverticulum of the transverse colon. Am J Gastroenterol. 1997;92(9):1567-1569. [PubMed: 9317094]

288. Shperber Y, Halevy A, Oland J, Orda R. Perforated diverticulitis of the transverse colon. Dis Colon Rectum. 1986;29(7):466-468. [PubMed: 3720460]

289. Peck MD, Villar HV. Perforated diverticulitis of the transverse colon. West J Med. 1987;147(1):81-84. [PubMed: 3424810]

290. Chen CW, Jao SW, Lai HJ, Chiu YC, Kang JC. Isolated rectal diverticulum complicating with rectal prolapse and outlet obstruction: case report. World J Gastroenterol. 2005;11(48):76977699. [PubMed: 16437704]

291. Plavsic BM, Raider L, Drnovsek VH, Kogutt MS. Association of rectal diverticula and scleroderma. Acta Radiol. 1995;36(1):96-99. [PubMed: 7833179]

292. Giffin HZ VII. Diverticulitis of the Rectum: A Report of Two Cases Operated Upon, One of them with Carcinomatous Degeneration. Ann Surg. 1911;53(4):533-537. [PubMed: 17862669]

293. EI S, OA M. Multipla diverticula of the colon. The Lancet. 1927;209(5412):1067-1074.

294. Walstad PM, Sahibzada AR. Diverticula of the rectum. Am J Surg 1968;116(6):937-939. [PubMed: 4972827]

295. Damron JR, Lieber A, Simmons T. Rectal diverticula. Radiology. 1975;115(3):599-601. [PubMed: 805459]

296. Alabiso ME, Grassi R, Fioroni C, Marano I. Iatrogenic rectal diverticulum in patients treated with transanal stapled techniques. Radiol Med. 2008;113(6):887-894. [PubMed: 18618073]

297. Halpert RD, Crnkovich FM, Schreiber MH. Rectal diverticulosis: a case report and review of the literature. Gastrointest Radiol. 1989;14(3):274-276. [PubMed: 2499497]

298. Frangi A, Gómez MA, Moya P, Arroyo A, López A. Rectal diverticulum resection by stapler device. Tech Coloproctol. 2014;18(4):403-404. [PubMed: 23224914]

299. WESTON SD, SCHLACHTER IS. Diverticulum of the rectum. Dis Colon Rectum. 1959;2:458464. [PubMed: 13844188]

300. Piercy KT, Timaran C, Akin H. Rectal diverticula: report of a case and review of the literature. Dis Colon Rectum. 2002;45(8):1116-1117. [PubMed: 12195201]

301. Jung SH, Kim JH. A case of solitary rectal diverticulum presenting with a retrorectal mass. Gut Liver. 2010;4(3):394-397. [PubMed: 20981220] 


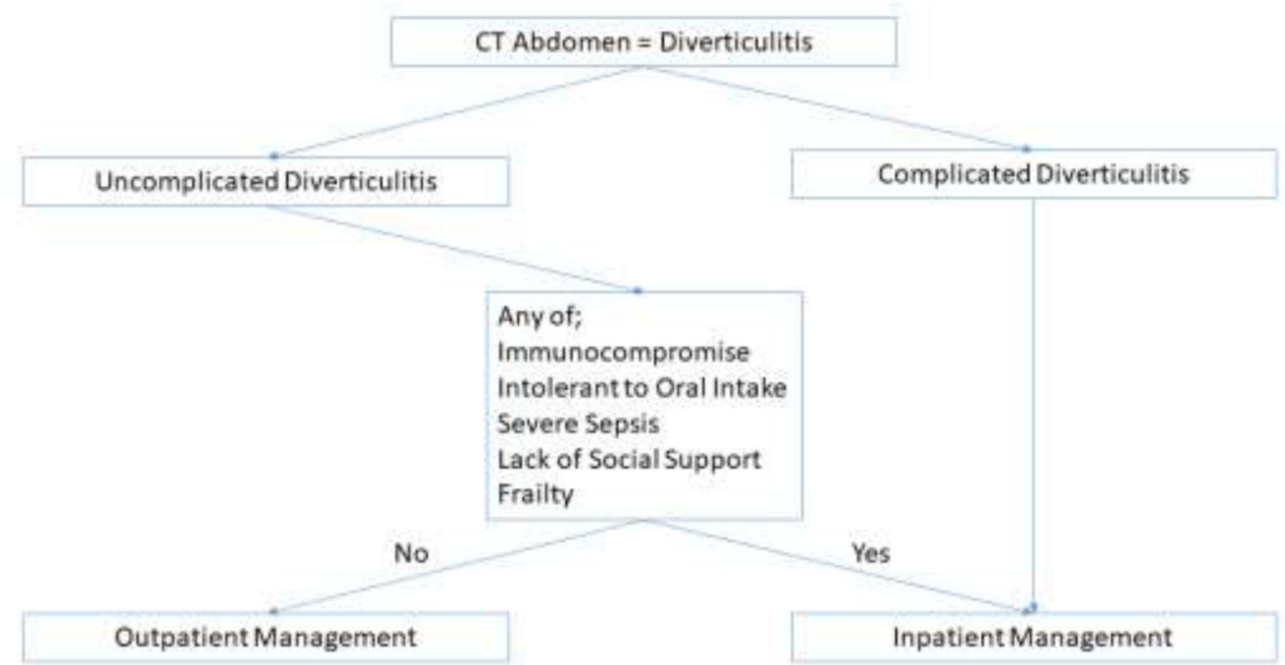

Figure 1-

Algorithm for selecting appropriate patients for out-patient management. 


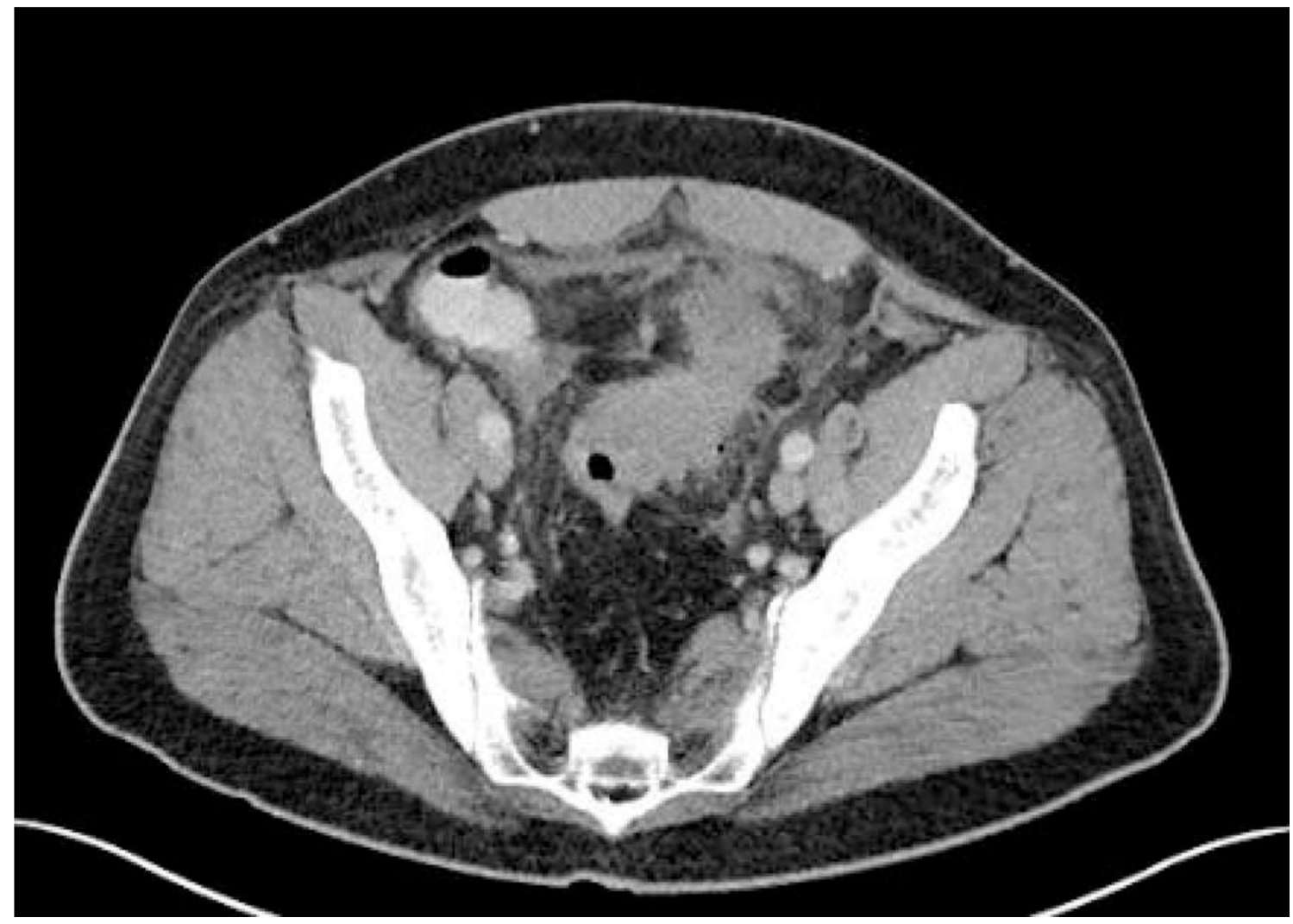

Figure 2.

Thickened sigmoid colon with adjacent mesenteric stranding consistent with acute uncomplicated sigmoid diverticulitis. 


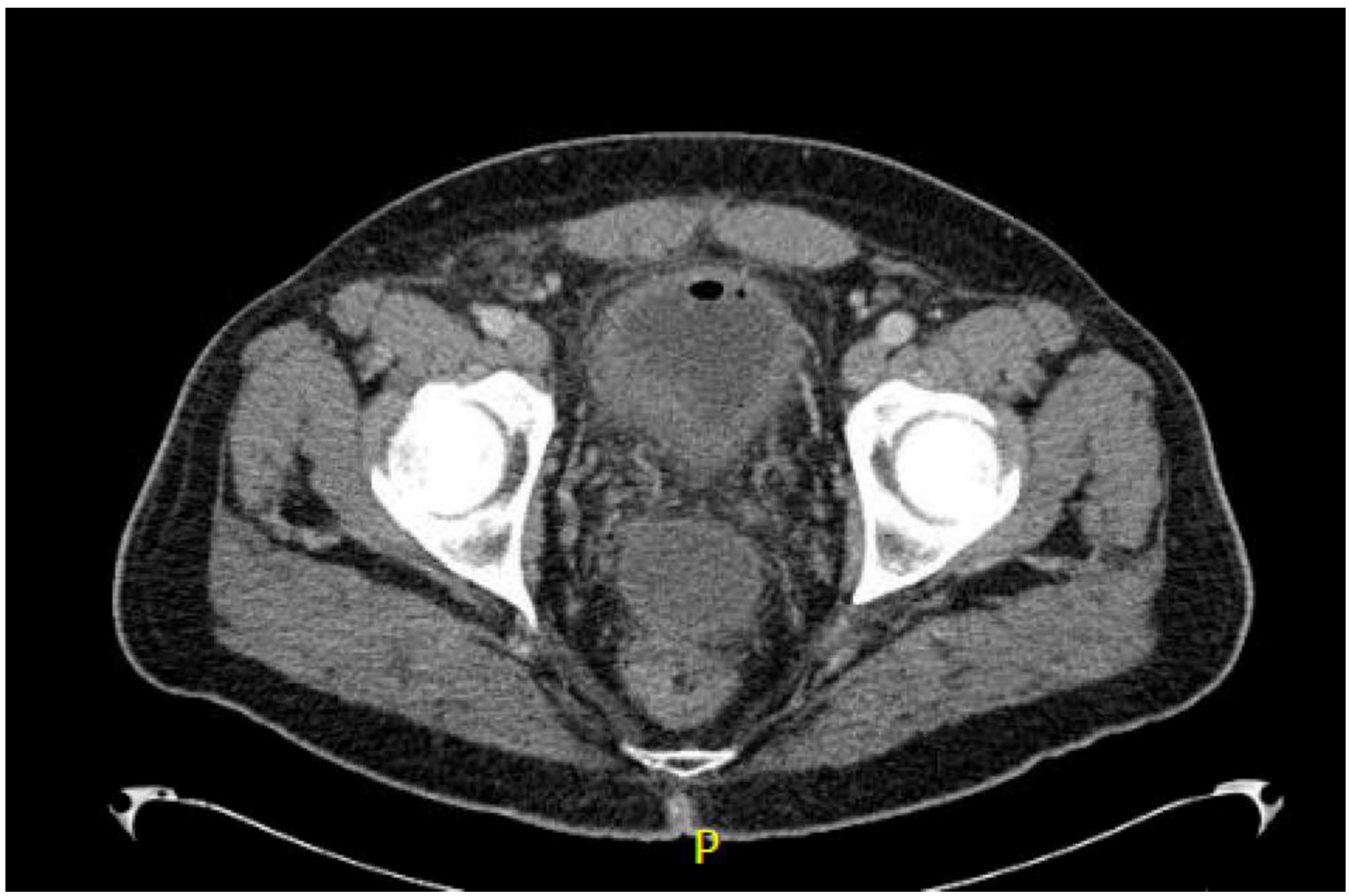

Figure 3.

Thickened bladder with air, consistent with colovesicular fistula. 


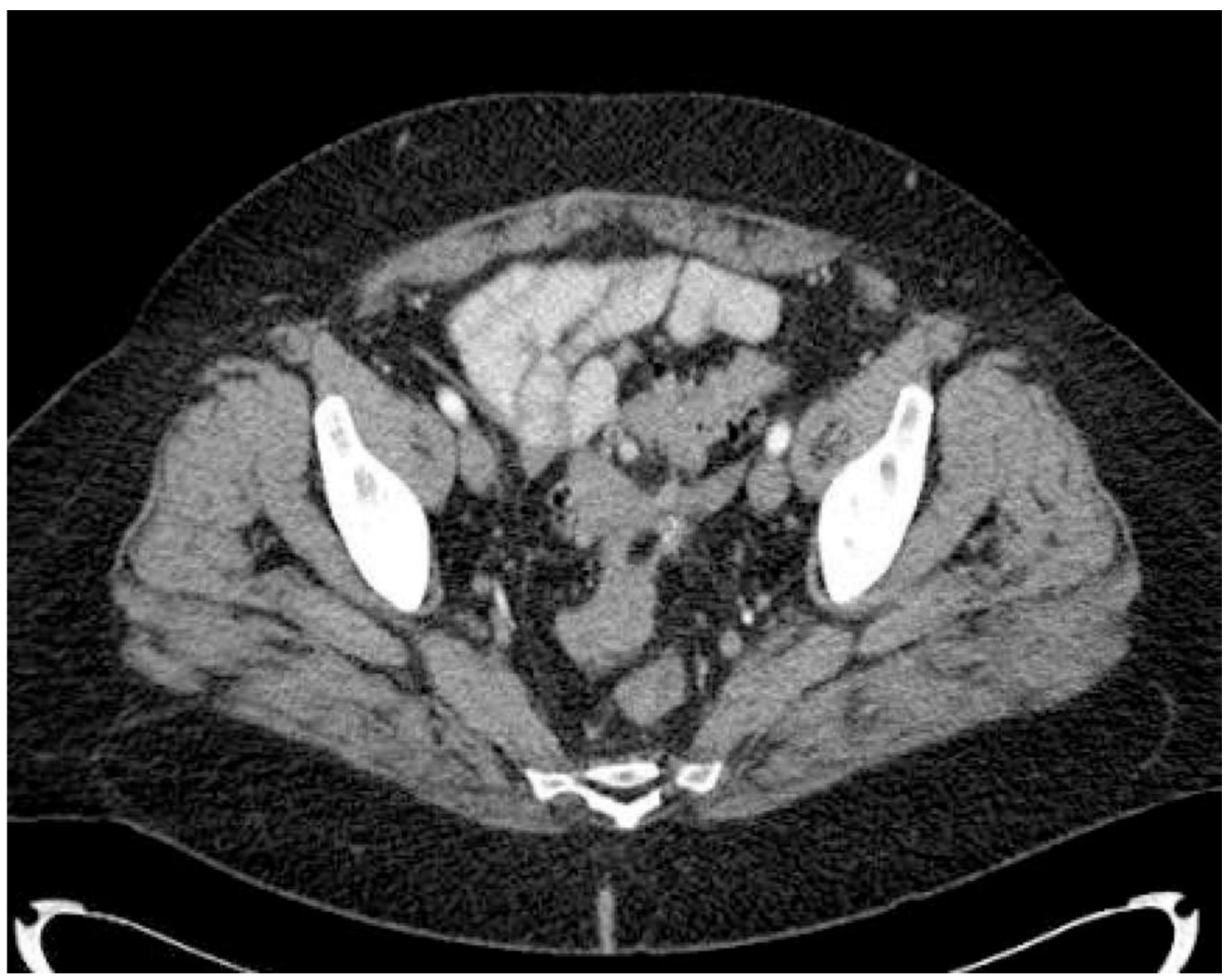

Figure 4.

Colovaginal fistula on a female patient with prior history of hysterectomy. Arrow marks fistulous tract. 

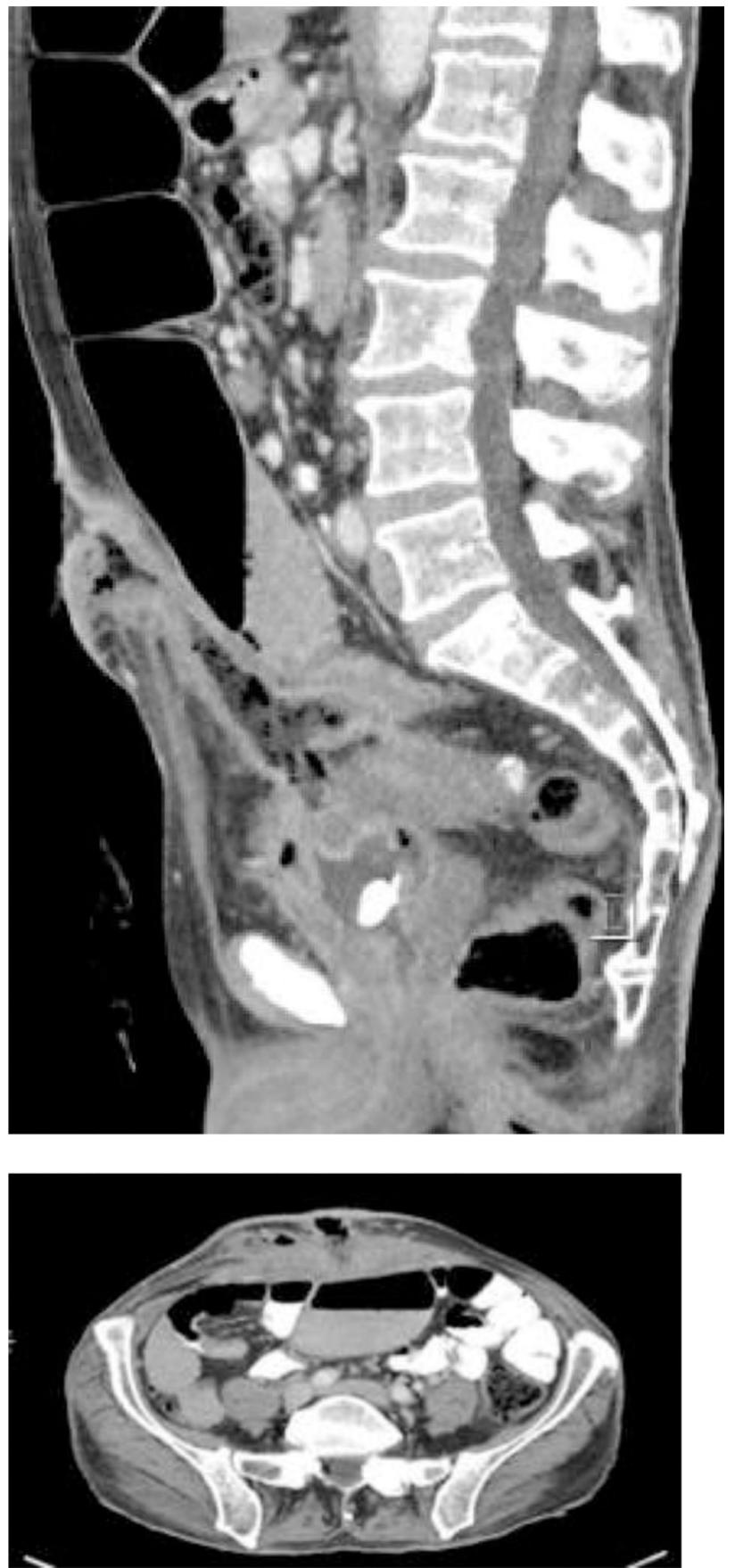

Figures 5\&6.

Colocutaneous fistula secondary to diverticular disease. Arrow marks fistulous tract and colocutaneous fistula. 


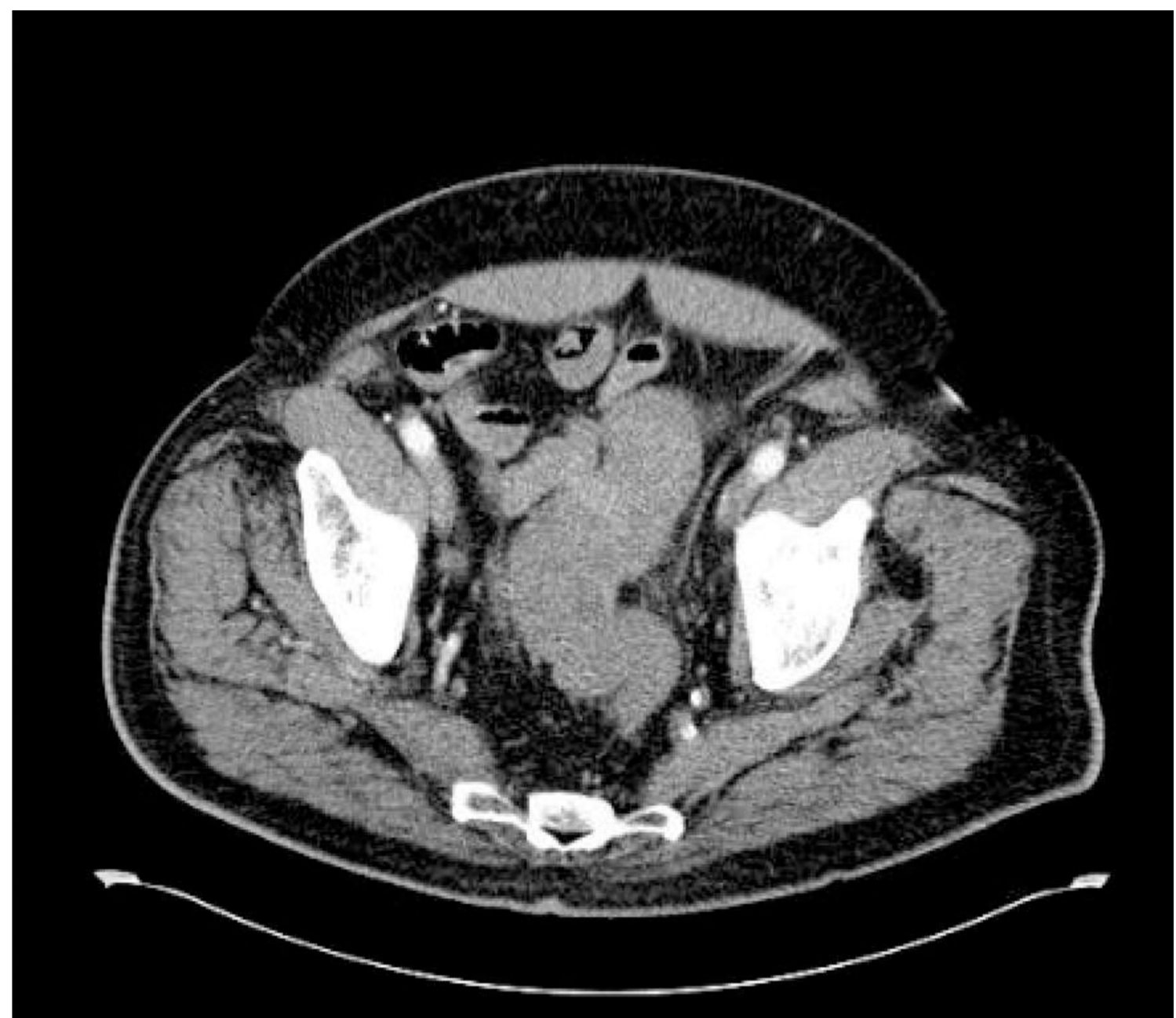

Figure 7.

Marked sigmoid colon thickening with luminal narrowing secondary to chronic sigmoid diverticulitis. 


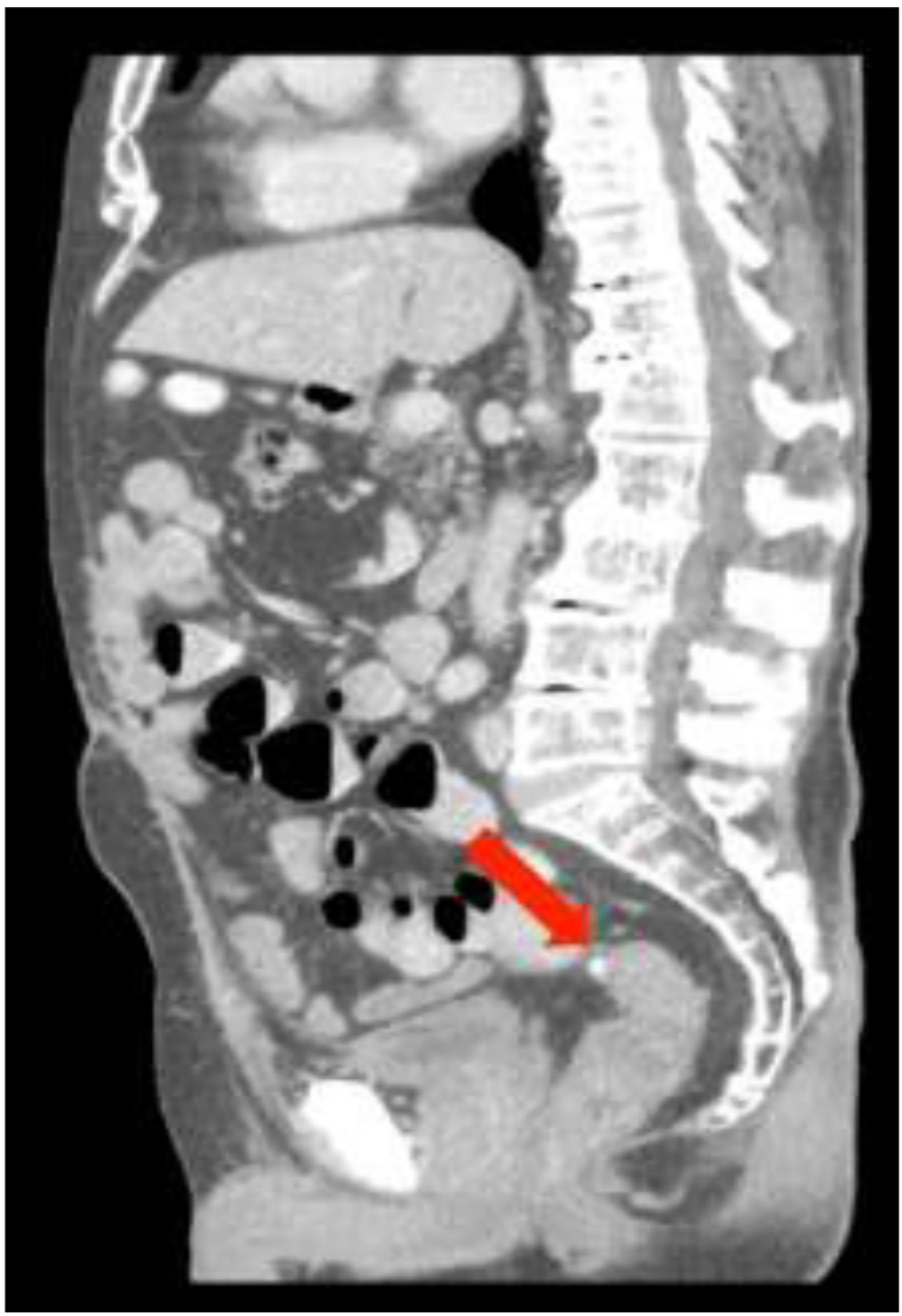

Figure 8.

CT scan, sagittal view. Red arrow marks the rectal stump. 


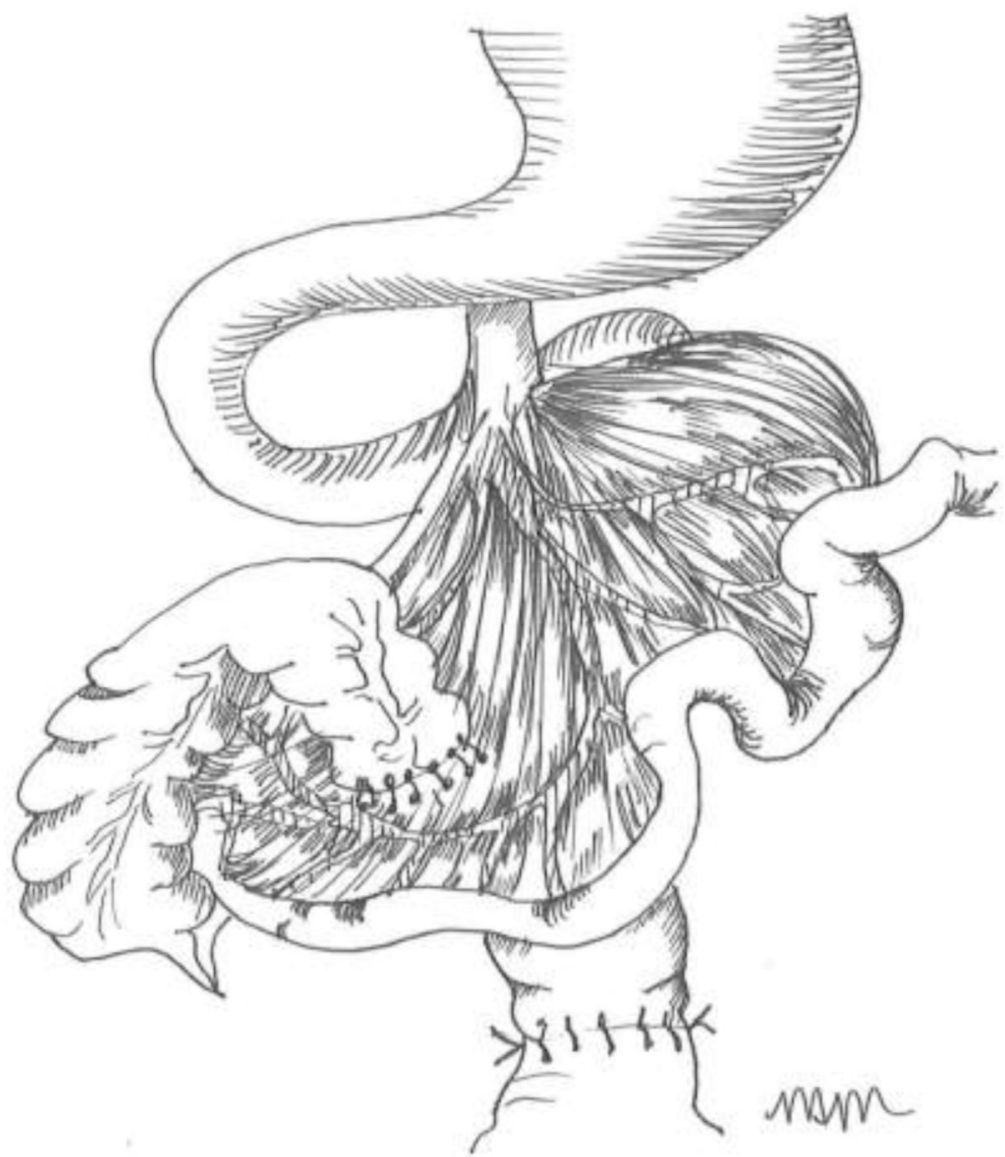

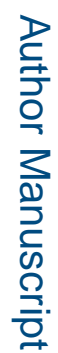




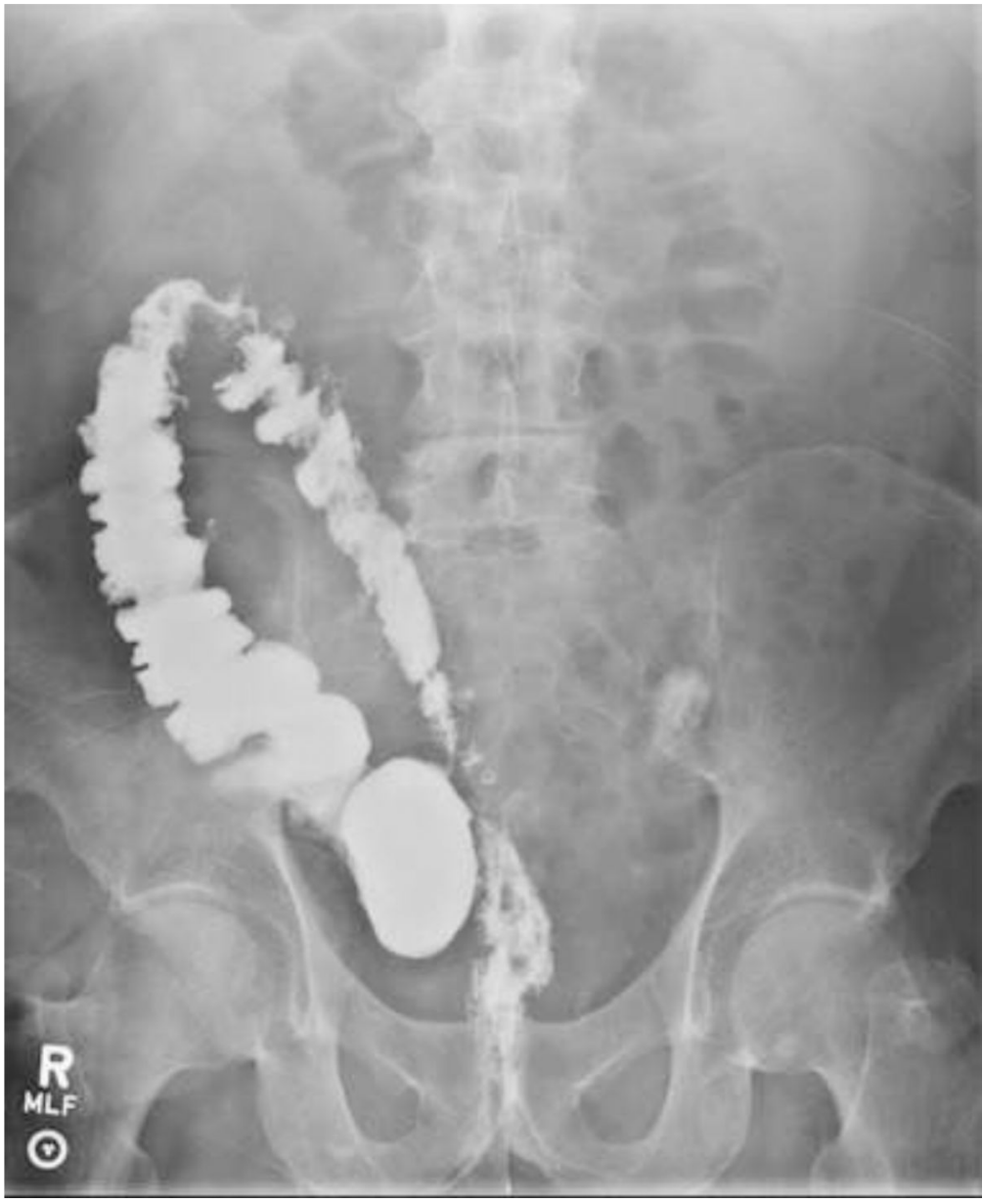

Figure 9.

Retroileal pull-through:

A) Anatomic Configuration

B) X-ray Contrasted Depiction of Anatomy. 
c.
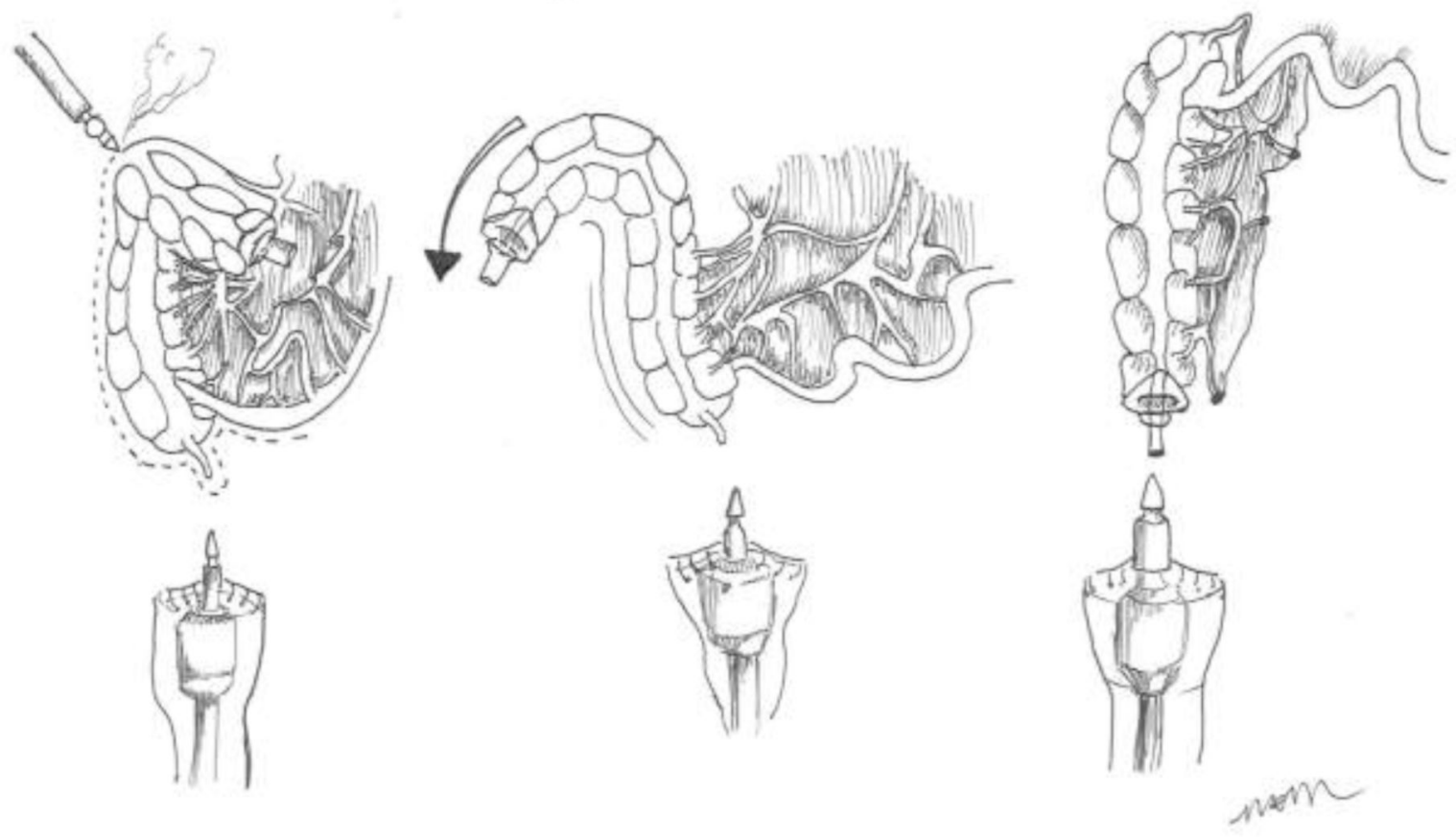

Figure 10.

The Deloyers Procedure 

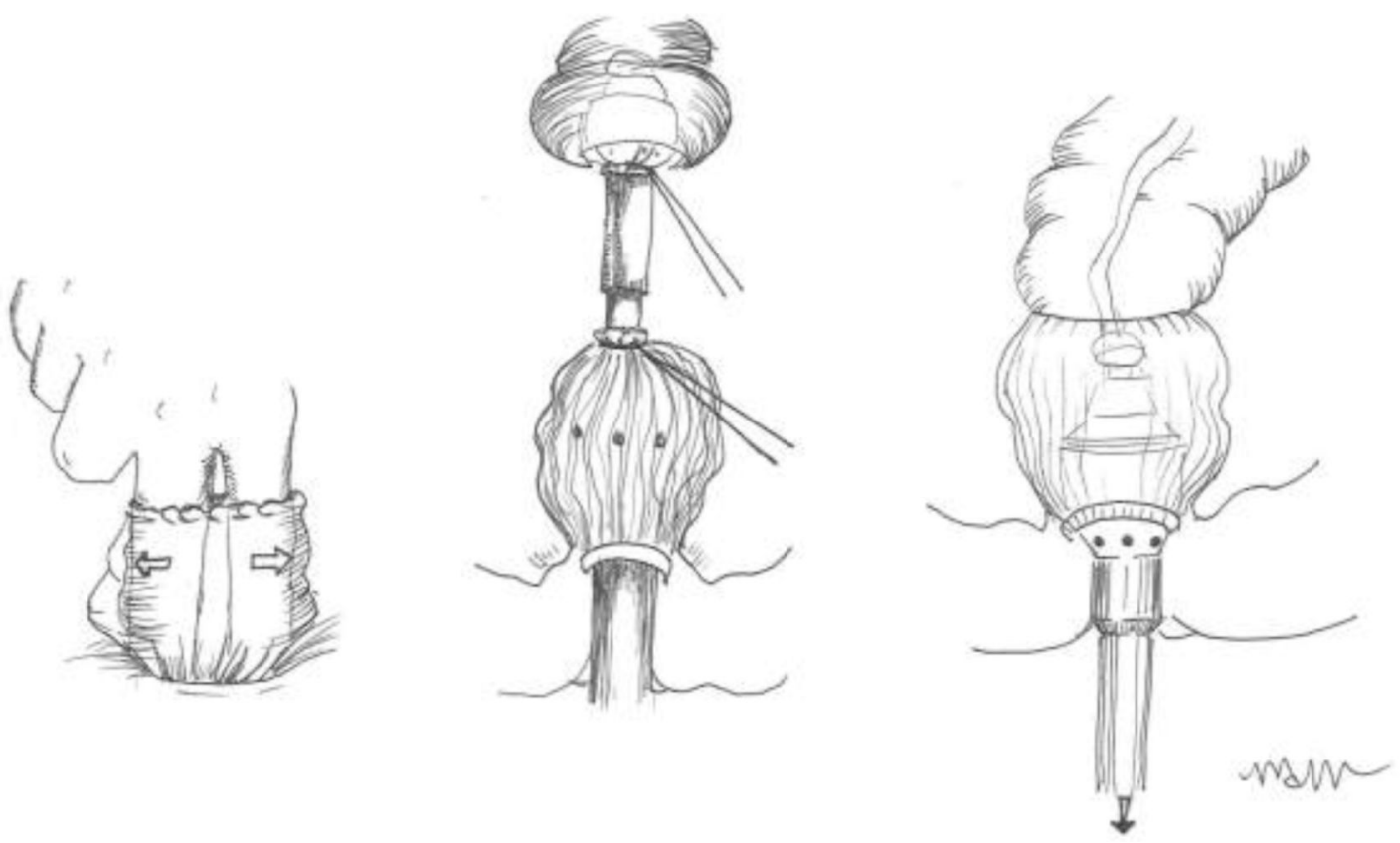

Figure 11.

Single stapled, double purse-string, end-to-end colorectal anastomosis. 

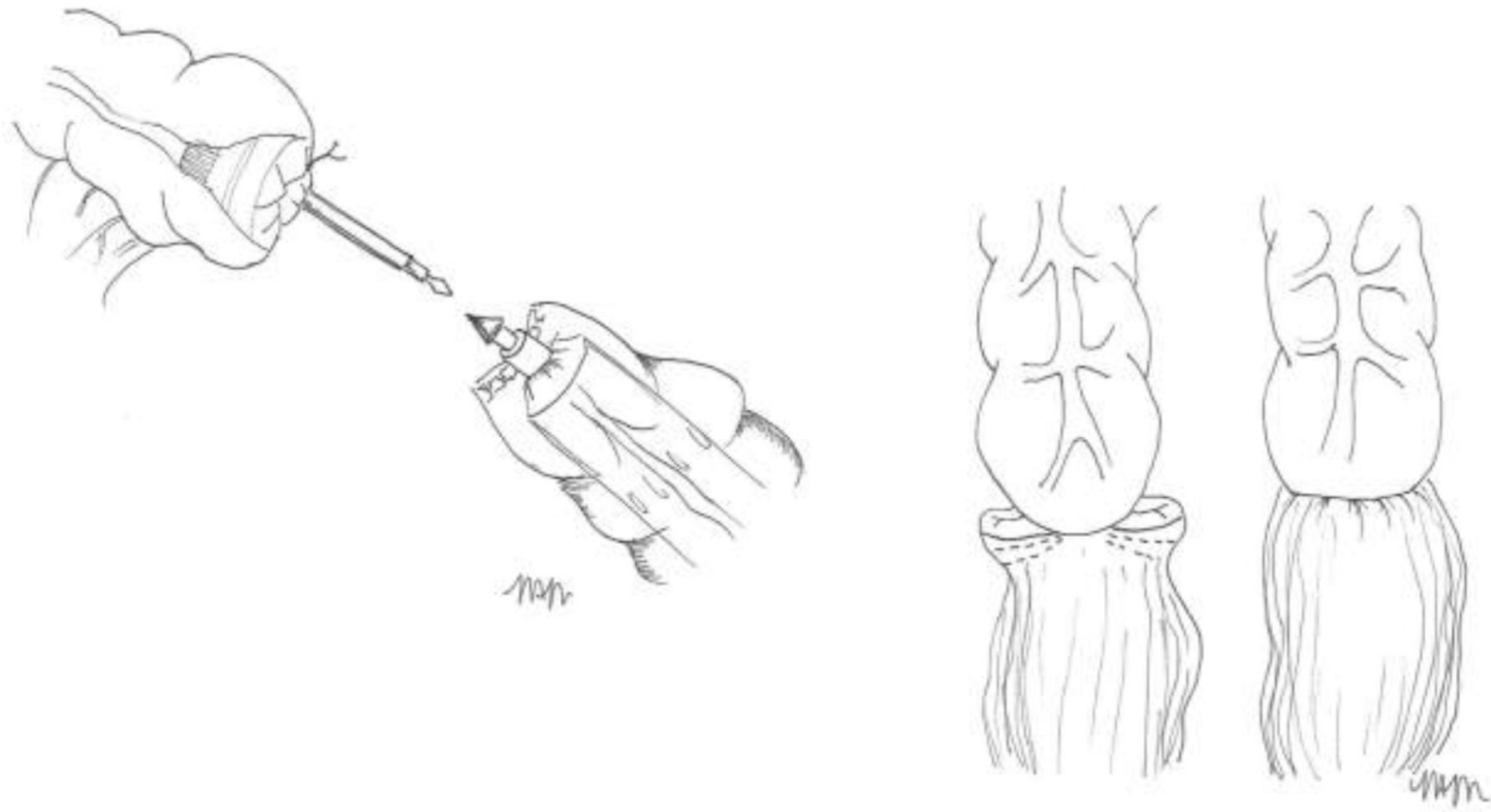

Figure 12.

The double stapled colorectal anastomosis. 

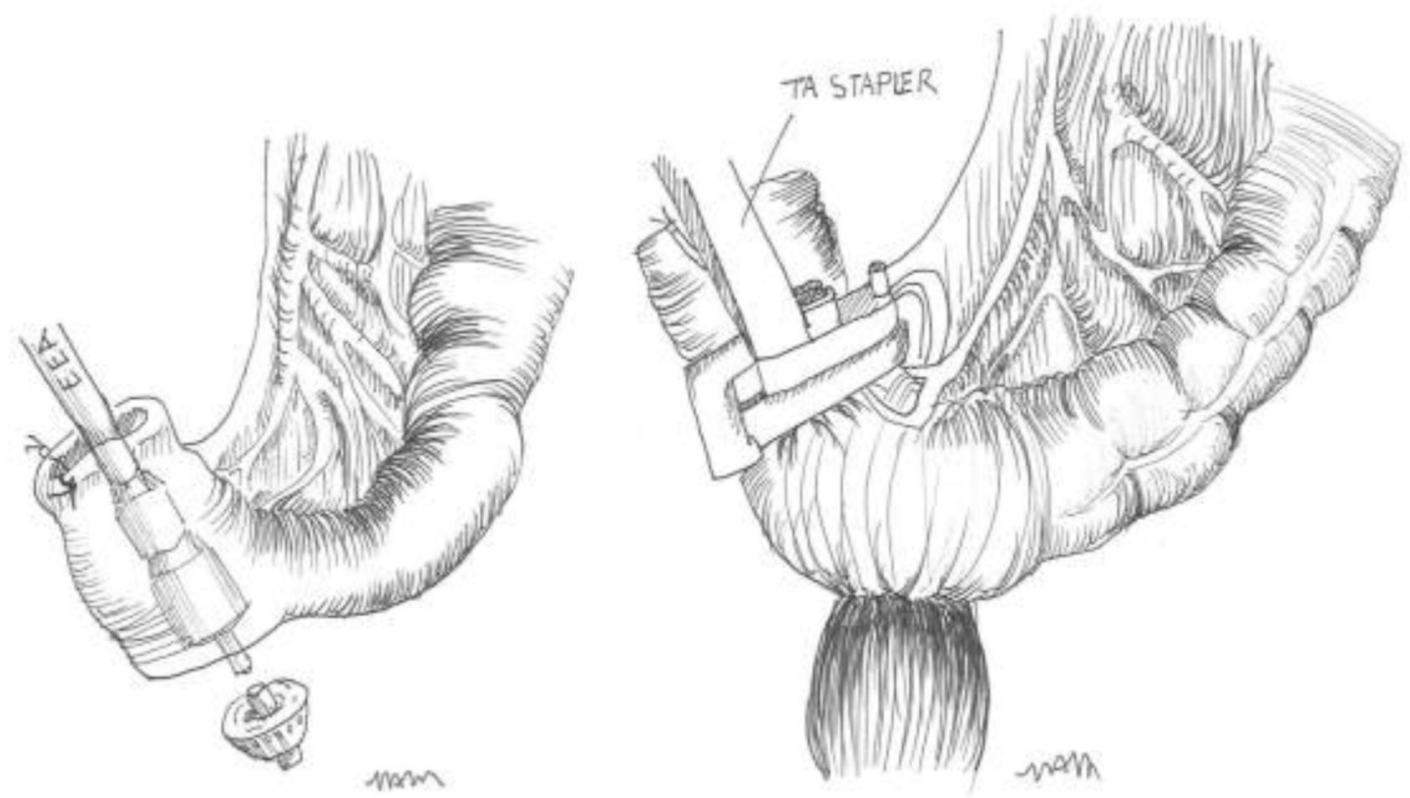

Figure 13.

The Upside Down stapled side-to-end colorectal anastomosis. 
Table 1:

Hinchey Classification of Diverticulitis

\begin{tabular}{|l|l|}
\hline Class & CT Findings \\
\hline Stage I & Localized abscess (pericolic abscess/phlegmon) \\
\hline Stage II & Pelvic intraabdominal or retroperitoneal abscess \\
\hline Stage III & Generalized purulent peritonitis \\
\hline Stage IV & Generalized feculent peritonitis \\
\hline
\end{tabular}


Table 2:

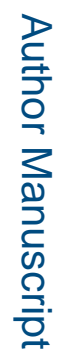

Ambrosetti Classification of Diverticulitis

\begin{tabular}{|l|l|}
\hline Class & CT findings \\
\hline Moderate disease & Localized sigmoid wall thickening $(<5 \mathrm{~mm})$ \\
& Pericolic fat stranding \\
\hline Severe disease & $\begin{array}{l}\text { Abscess } \\
\text { Extraluminal air } \\
\text { Extraluminal contrast }\end{array}$ \\
\hline
\end{tabular}


Table $2 b$.

Modified Hinchey Classification of Diverticulitis

\begin{tabular}{|l|l|}
\hline Class & CT Findings \\
\hline Stage 0 & Mild clinical diverticulitis \\
\hline Stage Ia & Confined pericolic inflammation/phlegmon \\
\hline Stage Ib & Confined pericolic abscess (within sigmoid mesocolon) \\
\hline Stage II & Pelvic, distant intra-abdominal or intraperitoneal abscess \\
\hline Stage III & Generalized purulent peritonitis \\
\hline Stage IV & Fecal peritonitis \\
\hline
\end{tabular}

